\title{
Lutte contre la pratique de l'excision au Mali: De l'approche santé à l'approche basée sur les droits de l'enfant-Rapport d'Evaluation du Programme du Centre Djoliba
}

Anta Fall Diagne

Population Council

Follow this and additional works at: https://knowledgecommons.popcouncil.org/departments_sbsr-rh

Part of the Demography, Population, and Ecology Commons, and the International Public Health Commons

How does access to this work benefit you? Let us know!

\section{Recommended Citation}

Diagne, Anta Fall. 2008. "Lutte contre la pratique de l'excision au Mali: De l'approche santé à l'approche basée sur les droits de l'enfant-Rapport d'Evaluation du Programme du Centre Djoliba." Dakar: Save the Children/Sweden and Population Council. 


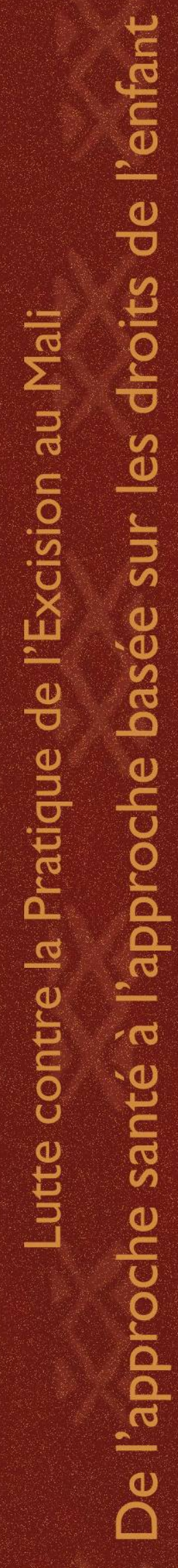

Rapport d'Evaluation du Programme du Centre Djolibe
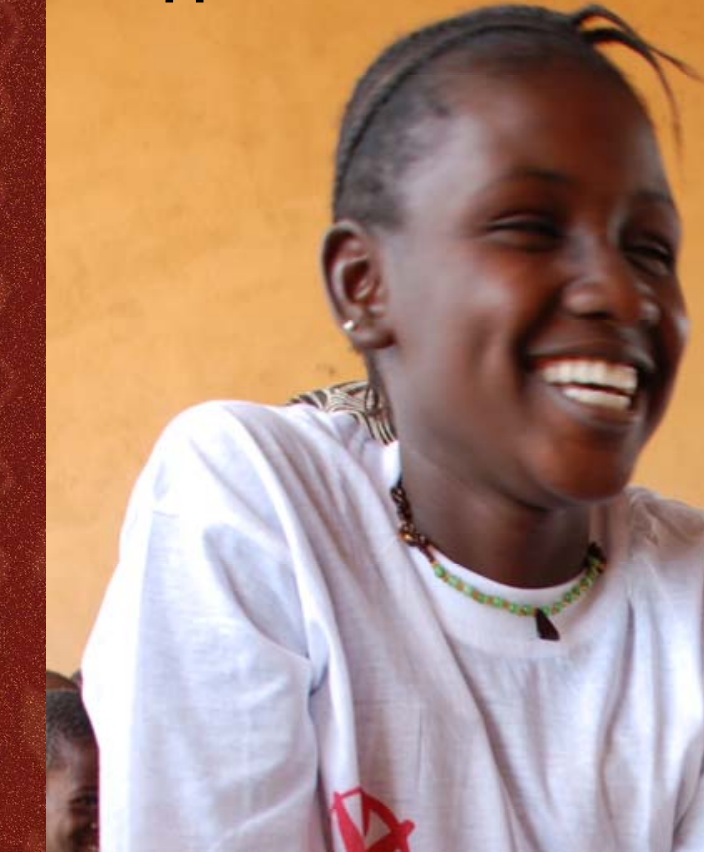
Save the Children Suède lutte pour les droits de l'enfant et apporte une amélioration durable dans la vie des enfants à travers le monde.

Notre vision est celle d'un monde où tous les droits de l'enfant sont réalisés. Save the Children œuvre pour :

- Un monde qui respecte et valorise chaque enfant

- Un monde qui écoute et apprend des enfants

- Un monde où tous les enfants ont de l'espoir et des perspectives d'avenir

Population Council est une organisation internationale, non gouvernementale et à but non lucratif qui réalise des recherches biomédicales, de sciences sociales et de santé publique.

Depuis plus de 50 ans, Population Council évalue et met au point des approches durables afin d'améliorer la santé et le bien-être des gens.

Numéro de Code : 10349

ISBN : 978-91-7321-321-9

(C) 2008 : Save the Children Suède, Bureau régional pour l'Afrique de l'Ouest et Population Council

Responsable du projet: Amé Atsu David, Save the Children Suède

Auteur : Anta Fall Diagne, Population Council

Equipe de recherche : Nafissatou J. Diop, Population Council ; Saran Boré Diakité, Consultante ;

Hélène Benga Nango, Consultante

Photos : Laurent Duvillier, Save the Children Suède

Conception graphique : Population Council

Imprimeur : Graphi-plus 
Lutte contre la pratique de l'excision au Mali

De l'approche santé à l'approche basée sur les droits de l'enfant

Rapport d'Evaluation du Programme du Centre Djoliba 


\section{Remerciements}

Tous nos remerciements vont à l'endroit de l'équipe du Centre Djoliba qui a bien voulu accepter de fournir toutes les informations et les renseignements qui nous ont été très utiles dans le processus de cette évaluation.

L'équipe adresse aussi ses vifs remerciements à toutes les personnes qui se sont investies dans la réalisation de ce travail. Leur engagement a contribué à son succès. Il s'agit d'abord de l'équipe du Bureau Régional de Save the Children Suède pour l'Afrique de l'Ouest, du Ministère de la Santé du Mali à travers sa Division de la Santé de la Reproduction, du Programme National de Lutte contre la Pratique de l'Excision du Ministère de la Promotion de la Femme, de l'Enfant et de la Famille et aussi de l'Honorable Député Daouda Touré du Réseau Malien des Parlementaires pour la Population et le Développement.

L'exécution de cette recherche a été facilitée par l'appui, la disponibilité et la participation de toutes les communautés rencontrées dans le cadre de cette évaluation.

Cette liste n'est certainement pas exhaustive, plusieurs autres personnes ayant apporté une précieuse contribution pour la réalisation de ce travail. Qu'elles trouvent ici l'expression de notre sincère reconnaissance. 


\section{Sommaire}

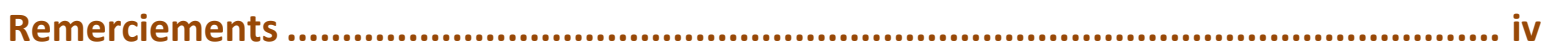

Sommaire

Liste des Abréviations ................................................................................................vii

Liste des Tableaux et Graphique.....................................................................................viii

Executive Summary ......................................................................................................

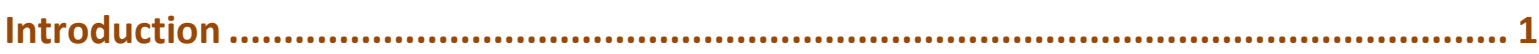

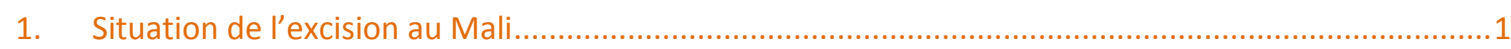

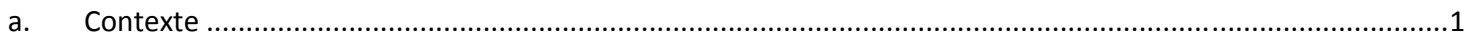

b. Cadre institutionnel des interventions en matière de lutte contre la pratique de l'excision au Mali.................2

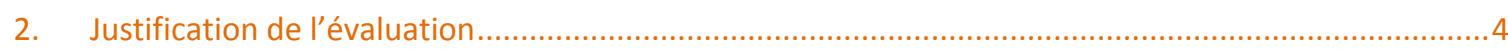

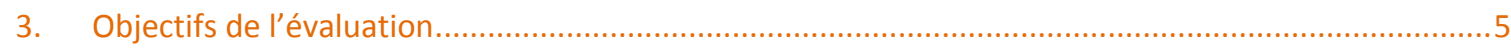

Méthodologie...............................................................................................6 6

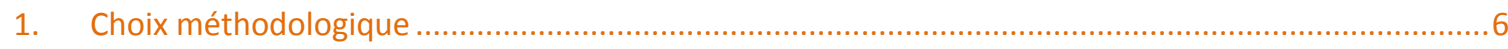

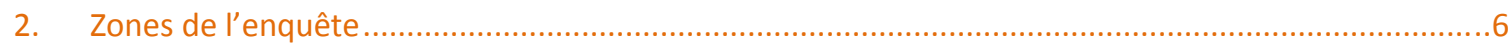

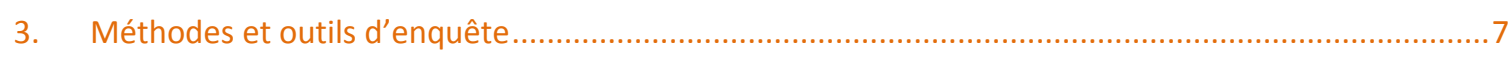

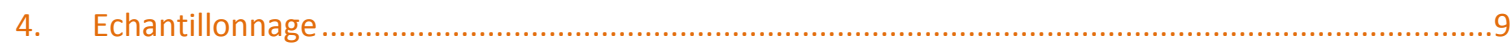

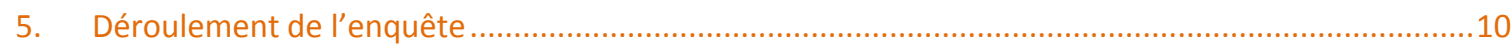

I- Présentation du Centre Djoliba ......................................................................... 12

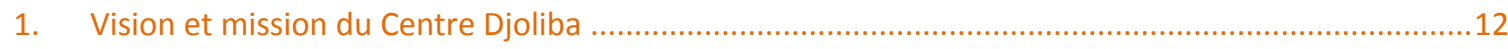

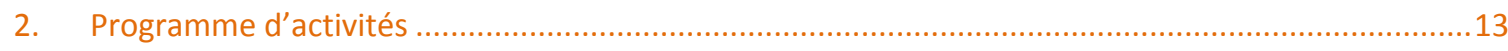

II- Processus d'intervention du Centre Djoliba .............................................................. 15

1. Stratégie de mise en œuvre du programme par le Centre Djoliba ............................................15

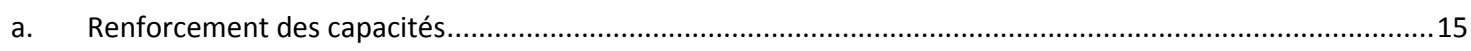

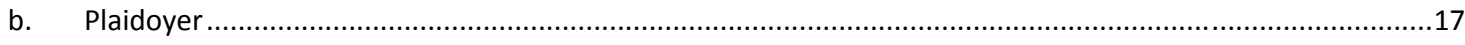

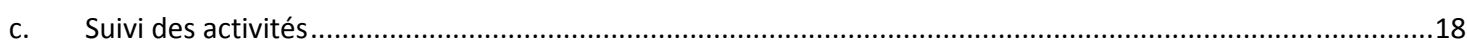

2. Stratégie de mise en œuvre du programme dans les communautés ........................................18

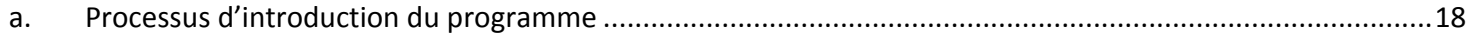

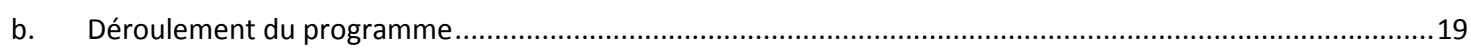

c. L'adhésion au programme et à ses messages .........................................................................................20

III- Résultats de l'évaluation .............................................................................. 21

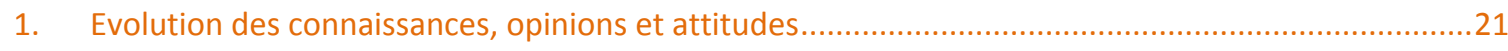

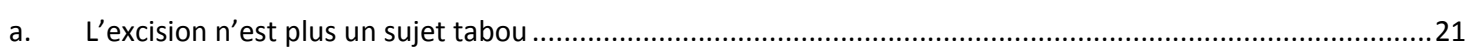




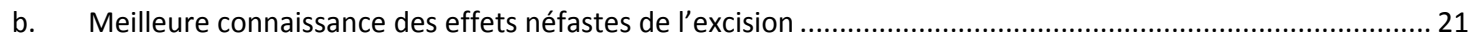

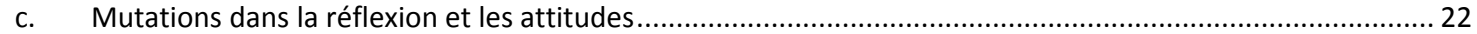

2. Description du processus d'abandon ou de régression ........................................................26

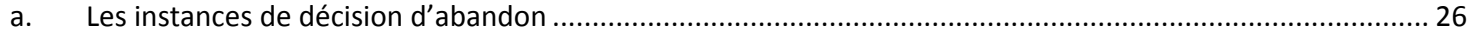

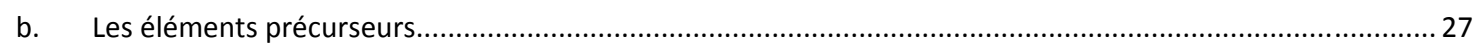

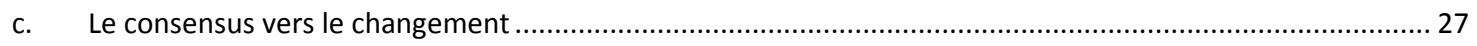

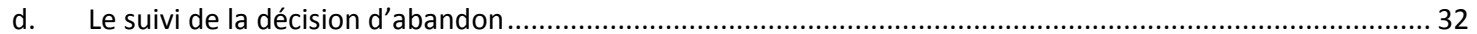

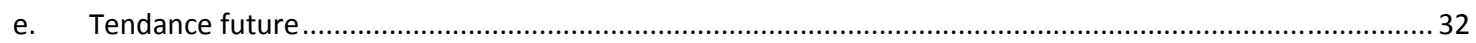

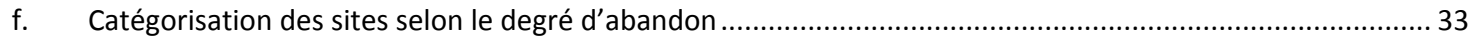

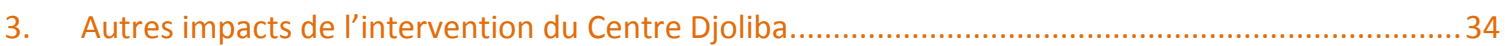

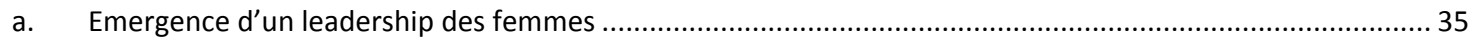

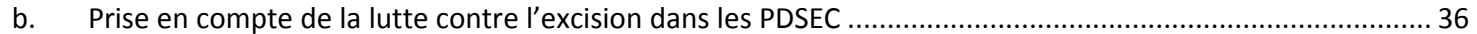

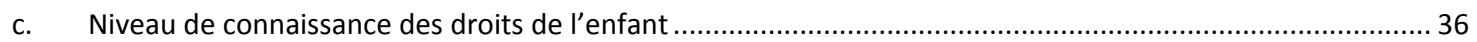

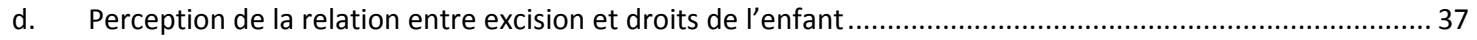

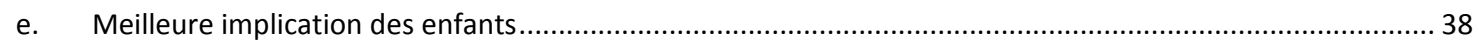

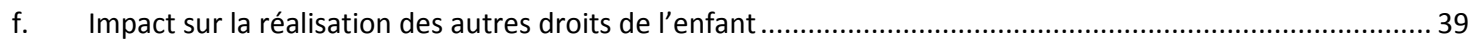

g. Impact positif sur l'économie des ménages ............................................................................................. 40

IV- Contraintes.............................................................................................42

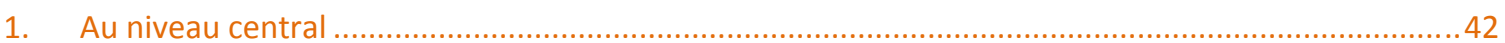

a. Collaboration avec le Ministère de la Santé et les autres secteurs ministériels ......................................... 42

b. Rapports entre le Centre Djoliba et ses antennes régionales ............................................................... 42

2. Au niveau de la mise en ouvre de l'approche basée sur les droits dans les communautés ..............43

a. L'absence de collaboration entre les différents acteurs dans les mêmes zones d'intervention ......................43

b. Insuffisance des moyens financiers, logistiques et matériels..................................................................... 43

V- Leçons apprises................................................................................44

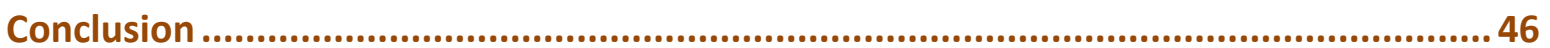

Recommandations......................................................................................48

1. A Save the Children Suède et aux autres partenaires ....................................................... 48

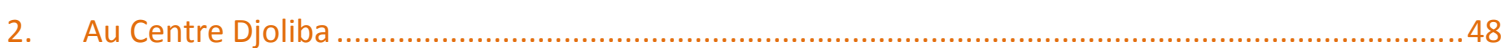

Références Bibliographiques............................................................................... 51 


\section{Liste des Abréviations}

\begin{tabular}{|c|c|}
\hline AGR & Activités Génératrices de Revenus \\
\hline AMPE & Association Malienne pour la Protection de l'Environnement \\
\hline AMPPF & Association Malienne pour la Promotion de la Planification Familiale \\
\hline AMSOPT & $\begin{array}{l}\text { Association Malienne pour le Suivi et l'Orientation des Pratiques } \\
\text { Traditionnelles }\end{array}$ \\
\hline APDF & Association pour le Progrès et la Défense des droits des Femmes \\
\hline ASDAP & Association de Soutien au Développement des Activités de Populations \\
\hline CAPN & $\begin{array}{l}\text { Comité National d'Actions pour l'Abandon des Pratiques Néfastes à la } \\
\text { Santé de la Femme et de l'Enfant }\end{array}$ \\
\hline CADBE & Charte Africaine des Droits et du Bien-être de l'Enfant \\
\hline CCFD & Comité Catholique contre la Faim et pour le Développement \\
\hline $\mathrm{CDE}$ & Convention des Nations Unies relatives aux Droits de l'Enfant \\
\hline CIAF & Comité Interafricain \\
\hline CLAPN & $\begin{array}{l}\text { Comité Local d'Actions pour l'Abandon des Pratiques Néfastes à la Santé } \\
\text { de la Femme et de l'Enfant }\end{array}$ \\
\hline COMAPRAT & Comité Malien Contre les Pratiques Traditionnelles \\
\hline $\mathrm{CPN}$ & Consultations Prénatales \\
\hline CRAPN & $\begin{array}{l}\text { Comité Régional d'Actions pour l'Abandon des Pratiques Néfastes à la } \\
\text { Santé de la Femme et de l'Enfant }\end{array}$ \\
\hline EDS & Enquête Démographique et de Santé \\
\hline GRAAP & Groupe de Recherche et d'Appui à l'Autopromotion des Populations \\
\hline IEC & Information Education Communication \\
\hline LIMAMA & Ligue Malienne des Imams et Erudits pour la Solidarité Islamique \\
\hline MGF & Mutilations Génitales Féminines \\
\hline ONG & Organisation Non Gouvernementale \\
\hline PNLE & Programme Nationale de Lutte contre l'Excision \\
\hline $\mathrm{RmL} / \mathrm{MGF}$ & Réseau malien de Lutte contre les Mutilations Génitales Féminines \\
\hline REMAPOD & $\begin{array}{l}\text { Réseau Malien des Parlementaires pour la Santé des populations et le } \\
\text { Développement }\end{array}$ \\
\hline RMCAEX & Réseau des communicateurs modernes \\
\hline SR & Santé de la Reproduction \\
\hline UNFM & Union Nationale des Femmes du Mali \\
\hline PDSEC & Plan de Développement Social, Economique et Culturel \\
\hline
\end{tabular}




\section{Liste des Tableaux et Graphique}

Graphique 1 : Situation géographique des zones d'étude.............................. 6

Tableau 1 : Localités de l'enquête..................................................................... 7

Tableau 2 : Nombre de focus groupes, d'entretiens individuels et de portraits

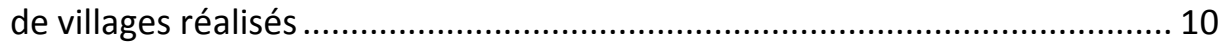




\section{Executive Summary}

\section{Introduction}

According to the latest Demographic and Health Survey (2006), Mali has one of the highest prevalence rates of female genital circumcision (FGC). At the same time, the involvement of Mali in the fight against the practice of excision was demonstrated early on, dating back to the 1950s and the African Women's Congress (Congrès des Femmes Africaines) which was held in Bamako in 1959. This involvement was reinforced in 1996 with the creation of the Commission for the Promotion of Women (Commissariat de la Promotion des Femmes) which established the National Action Committee for the Abandonment of Harmful Traditional Practices Affecting the Health of Women and Children (CAPN). The National Program against Excision was created in 2002. In Mali, approximately $80 \mathrm{NGOs}$ are involved in the fight against the practice of excision and the Centre Djoliba is one of the pioneers.

Since the 1980s, Save the Children Sweden has supported activities aimed at eliminating the practice of FGC organized by the Centre Djoliba in Mali. A turning point was noticed in 2004 with the introduction of a rights-based approach. This new strategy, which places the focus on human rights, involves placing women and children at the center of the debate, considering them as social actors, while at the same time promoting community empowerment so as to find a local response to the harmful traditional practice of female genital circumcision.

\section{Methodology}

A qualitative study was conducted among the communities concerned in the regions of Koulikoro, Sikasso, Ségou and Kayes. Twelve villages in the areas where the Centre Djoliba is intervening were selected for the study by rational choice, taking into account the particularities of each village. Then, during a onemonth period, field data was collected by a multi-disciplinary team. In total, the survey reached some 200 people across the various investigation methods employed including focus groups, in-depth interviews and village profiles.

\section{Centre Djoliba}

Established in 1964, the Centre Djoliba is a structure that provides capacity building to development actors and stakeholders to help reinforce their intervention capabilities. The Center is comprised of two regional branches, one located in Sikasso (2002) and one in Ségou (2004) and works with NGOs, women associations, youth groups, village communities and local authorities. 
The Centre Djoliba has a vision of FGC as a practice that encompasses myriad dimensions: anthropological, ethnological, religious, health and human rights. In the context of its program focusing on the promotion of Reproductive Health, the Education Project for the Abandonment of Excision and the promotion of the rights of women and children introduced a new orientation of the Center moving from a health-based to a rights-based approach. In fact, the issue of female genital circumcision was first considered as an issue affecting women and the activities envisioned by the Center were only aimed at targeting women's groups and associations. Starting in 2004 the Centre Djoliba adopted the rightsbased approach advocated by Save the Children Sweden. Since that time, the issue of FGC has been viewed as a violation of the rights of the child, in particular the girl child, and of women.

\section{Documentation of the Process}

\section{Program Implementation by the Centre Djoliba}

The implementation process began with the training of 52 Community Relays who were trained in the materials related to the rights-based approached. Following this training they were sent to the villages targeted in the Centre Djoliba intervention to organize community awareness sessions including at elementary and high schools. The Centre Djoliba also conducted training for key stakeholder groups that were deemed essential to efforts aimed at eliminating the practice of FGC at the community and national level. These include community and village authorities, religious leaders, key opinion leaders, traditional and modern communicators, teachers, women and youth associations and groups, health workers, excisers, parliamentarians and officials from various government institutions.

In addition, the Centre Djoliba has played and continues to play an important role in the advocacy for the abandonment of the practice of excision at the national and the community level. At the national level the Centre Djoliba works in collaboration with Malian Parliamentarians to progressively achieve the adoption of an anti-excision law. Similarly, the Center conducts capacity-building activities for the National Program for Fight against Excision in Mali specifically in the area the approach based on the Rights of the Child.

\section{Implementation at the Intervention Sites by the Community Relays}

The Centre Djoliba activities in a village begin with an environmental analysis followed by information meetings with the local authorities and government representatives. Community meetings are then organized with the men, women, and youth to introduce them to the program content and objectives and to discuss issues related to their participation. This slow, but progressive approach, is preferred by the Centre Djoliba as a way of not jolting existing habits and beliefs. 
Next, the trained Community Relays, including the children and youth, organize activities aimed at raising awareness in the communities about the harmful effects of female genital circumcision placing specific emphasis on the Rights of the Child including their right to good health, physical integrity and protection from all forms of violence and abuse. Other related topics are addressed during these sensitization sessions in a holistic manner that is tailored to the socio-cultural realities of the specific intervention site.

If it has been easy in certain villages to gain their accord to participate in the program, in many others there has been quite a bit of reluctance, particularly on the part of some Muslim religious leader groups as well as among some of the excisers and their apprentices.

\section{Results}

\section{Evolution of Knowledge, Opinions and Attitudes}

- The subject of female genital circumcision is no longer taboo

Several respondents, notably the Community Relays who are, in many ways, at the forefront of the mobilization effort, have underscored the fact that the subject of FGC and talk about community abandonment of the practice is today discussed far more openly in the villages.

- Improved knowledge about the harmful effects of excision

Thanks to the interventions conducted in the villages by the Community Relays and the various strategic groups, the campaigns aimed at raising public awareness conducted via the radio and other mediums, the communities have had access to a vast amount of information about the different aspects of FGC, most particularly its harmful effects. Many of the respondents highlighted the long list of complications and negative consequences linked to excision at the medical and psycho-social level for the girl child and women.

- Changes in thinking and attitudes

Access by the populations to more comprehensive and detailed information about the traditional practice of excision has resulted in a profound change in thinking and attitudes. There is equally a calling into question about the validity of the practice.

Indeed, if in the past men did not have a lot of experience with non-excised women, today, as there are more and more married women who have not been circumcised who are fulfilling their marital duties and sexually satisfying their husbands, the latter are starting to state their preference for non-excised girls. Thus female circumcision once considered as a condition sine qua non for marriage for various reasons is appearing to be less and less an obstacle to the sexually harmony of couples and thus as a threat for the stability of the home and families.

\author{
Thus female \\ circumcision once \\ considered as a \\ condition sine qua non \\ for marriage for various \\ reasons is appearing to \\ be less and less an \\ obstacle to the sexually \\ harmony of couples and \\ thus as a threat for the \\ stability of the home and \\ families.
}


Some (religious leaders) have begun, as a result of an increased awareness, to begin to question and analyze anew the predicates or religious dogma before declaring that excision is not a required religious obligation.
These practitioners (excisers) cited several reasons for stopping among them the knowledge gained from the trainings and the IEC sessions which they have found beneficial, the fear of reprisals, and the fact that their services are no longer being solicited in certain villages following the adoption of the declaration of abandonment of the practice of FGC by various villages.

\section{Abandonment or Decline of the Practice}

There is observed a total abandonment or a sharp decline in the practice of FGC. Thus it is accepted by all categories of respondents, regardless of the village, that the practice of female genital circumcision is on the decline. Many of those interviewed see this trend as a result of the interventions of the Centre Djoliba. The consensus on the abandonment of female genital circumcision is generally a precursor to questioning the raison d'être of this practice, owing to access to a broader awareness about the extent of its negative consequences.

The consensus around the abandonment of the practice of excision is usually followed by efforts to adhere to this decision and to monitor the evolution of this abandonment in the community. The monitoring is done formally or informally, depending on the specific case. In some localities where the abandonment of the practice is widespread the villagers have set up ad hoc committees for monitoring and policing for the purpose of ensuring that the declaration of abandonment of the practice is respected. In other villages, it is felt that there is no need to establish such committees. The involvement of the village chief is closely monitored by the populace which is very much attached to respecting the community decisions and forums.

\section{- Participation and Involvement of Religious Leaders}

If at the beginning, the majority of religious leaders admitted their lack of knowledge about the harmful effects of the practice of female genital circumcision and demonstrated their reluctance to abandon the practice, some have begun, as a result of an increased awareness, to begin to question and analyze anew the predicates or religious dogma before declaring that excision is not a required religious obligation. And once doing so, these religious leaders have joined in the fight against FGC and have themselves become themselves actors in the sensitization of members of their communities. The position of these religious leaders demonstrates that it is possible to have the approval, participation, and involvement of this group in raising awareness and strengthening their capacities on the issue as well as other members of the community.

\section{- Mobilizing Excisers against the Practice of Excision}

Le Centre Djoliba conducted sessions for former excisers in capacity building, Information, Education and Communication (IEC) and also provided support and organized them into an association. The majority of the informants noted that these practitioners cited several reasons for stopping among them the knowledge gained from the trainings and the IEC sessions which they have found beneficial, the fear of reprisals, and the fact that their services are no longer being solicited in certain villages following the adoption of the declaration of abandonment of the practice of FGC by various villages. These former excisers are now involved in the fight against the practice of excision. The ones interviewed declared very strongly that they will never again resume their former activities. 
The loss of the income, something that is often cited as a reason for resuming the practice (by excisers) has also been considered in the program. To address the issue of the loss of income in terms of financial gains received from the practice of excision, the former excisers have, with the financial support of other development partners, organized themselves and joined with other women's associations to start new income generating activities. Thus the excisers have other income sources and some are recruited as facilitators by various NGOs and the Centre Djoliba.

\section{Additional Impact Centre Djoliba Intervention}

\section{- Emergence of women's leadership}

The emergence of women's leadership is the result of the training and the capacity building of women by the Centre Djoliba, the result of which has been an increased awareness among women of their place in the community and their potential for action especially when they come together as a group..

\section{- Inclusion of the fight against FGC in the PDSEC}

In some locales excision has been included in local development action plans (Economic, Social and Devlopment action Plan) signifying the important involvement of administrative authorities and ensuring the sustainability of the activities aimed at fighting this practice.

\section{- Level of knowledge about the Rights of the Child}

Information on the Declaration of the Rights of the Child and its content was distributed and has been widely circulated in the villages and towns targeted in the intervention. Certain child rights such as those pertaining to health, education, school enrollment, civil registration of birth certificates, food, breastfeeding (exclusively), monitoring, care and supervision of the child, are cited more frequently than others. The changes induced by the introduction of the rightsbased approach are being incorporated in terms of improving knowledge about these rights but also in a genuine attempt to put them into practice in daily life.

- Perceptions about the relationship between the practice of excision and the Rights of the Child

The majority of the people who have been exposed to the various interventions do make the connection between the Rights of the Child and excision and they believe that excision is actually an obstacle to the full enjoyment by young girls, young women and women of their rights.

\section{- Increased Involvement of Children}

The intervention of the Centre Djoliba, more specifically the introduction of a right-based approached in the villages has led to changes, one of which has been an improvement in addressing the need of children. Their involvement in the activities in the fight against the practice of excision is effective but their involvement in the decision-making, which is a longer process, is a more gradual process. The youth are involved in villages where Support Clubs have been 
established in the schools. This framework allows the children to be trained and then eventually be able conduct activities themselves among their classmates, friends, teachers and others members of the community.

\section{- Positive Impact on Household Incomes}

Women have benefitted from financial support in the form of micro-credit loans that have enabled them to undertake income generating activities (market-garden farming, small trade, etc.) and thus to help improve their daily lives. In some villages, the communities have organized to pool their own funds to build schools, for example.

\section{Constraints}

\section{- At the Central Level}

The collaboration between the Centre Djoliba and the DSR (Division de la Santé de la Reproduction) of the Ministry of Health suffered from a lack of involvement of the DSR in the activities of the Centre Djoliba, something that could lead to non-compliance with the Norms and Standards of Reproductive Health in the tools used and a lack of reporting of data to the Ministry of Health. Shortcomings were also found regarding the relationship between the Center Djoliba and its branches in Segou and Sikasso in the operation and division of labor in terms of a lack of autonomy of the branch offices in the implementation of the Reproductive Health program including the fight against FGC.

- Implementing the Rights-based approach at the Community Level

The lack of collaboration between the different actors in the same intervention zone, the widespread poverty of the population and the lack of sufficient financial, logistical and material means constitute the major non-negligible constraints and make the geographic coverage of the Centre Djoliba in the remote rural communities very limited.

Moreover, some respondents deplored the adverse socio-economic conditions which constitute a serious handicap to the implementation of certain aspects of the program in particular in the area of providing care for children.

\section{Lessons Learned}

- Success Factors

- The methodology applied by the Centre Djoliba to go to the populations and introduce the subject of FGC as a policy of "small steps" that are tailored and adapted to each community;

- The teaching tool used in the interventions, namely the GRAAP Method, is suitable for a non-literate population employing visual images of scenes from daily life that are easily understood;

- The commitment of the Community Relays, their enthusiasm and their skills helps enhance the quality of their work with the populations;

- Women have more opportunity to emerge economically, especially when they come together in well-structured groups or associations. This allows 
them to be more autonomous in seeking funds from other donors and thereby providing assurance for sustainability of their activities.

The Centre Djoliba plays the role of intermediary or a facilitator between the populations it supervises and other projects allowing, for example, access to women to funding sources for their income generating activities.

- Risks Factors

- The context of poverty constitutes a major problem and could be an obstacle to the application and enforcement of the rights. The following statement shows that beyond the issue of abandoning the practice of excision, one of main worries for the populations is the fight against poverty:

"The reduction of poverty through income generating activities for the development of women is still a dream here in Karo." (Non-participating woman, Karo)

- The increasing workload for the program head of the Centre Djoliba and the fact all the main aspects of program implementation are headquartered at the Centre Djoliba runs the risk of there being a rupture in the program if this person should leave the Centre Djoliba for one reason or another. Thus there is a need to document and enhance the work of the Centre Djoliba in the field.

- The lack of coordinator among the different donors in the field runs the risk of sending out conflicting messages to the populations. In fact, the weakness of a program supported by one donor could serve to prejudice the unfolding of another program supported by a different donor.

- The fact that the Centre Djoliba receives funds from several different donors who promote different approaches could serve to hinder the operation of some of its activities in the field.

- The partial-engagement or short-time commitment by donor partners could trigger risks linked to sustainability of activities and the credibility of the Centre Djoliba among the populations. For example, in the event of a sudden termination of a program due to the discontinuation of funding by one of the funding donors of the Centre Djoliba.

\section{Conclusion}

The purpose this evaluation has been to assess the impact of the program of the Centre Djoliba on the lives of the communities selected and to examine the section using the human-and-child rights based approach in the major changes in the target communities. It was found that this approach has led to an improved understanding about these rights and that there is a willingness on the part of the communities to try to integrate the knowledge gained about these rights into their daily lives. In fact, some groups have realized that the Rights of the Child are linked to various areas affecting the growth, development and full blossoming of the child in terms of education, health, protection, recreation, etc. The 
communities have been sensitized about the fact that children should receive more care, attention and protection and actions have been noted that reflect an attempt to integrate this knowledge into daily living. But the adverse socioeconomic environment constitutes a serious obstacle to the full realization of these rights. All the same, the most important element is the appearance in these communities of arguments against female circumcision and non-circumcision. It is a new phenomenon which proves that the practice has been shaken. The testimonies of marriages with non-circumcised women and the benefits that result is the reflection of a social change.

\section{Recommendations}

The human-and-child rights-based approach is a new and interesting approach which should be conducted over the long-term if one wants to obtain a lasting change in the populations. The following recommendations have been drawn from this evaluation:

\section{For Save the Children Sweden and other Partners:}

- Develop a coherent and holistic training manual for use in the practical application of the human-and-child rights-based approach that is tailored to the context of FGC including the different modules on rights, reproductive health, and development activities;

- Improve the educational tools by including in them the text of the Declaration Rights of the Child and translating these tools into the main local indigenous languages;

- Support advocacy at the government level;

- Allocate more funds to those intervening in the fight against the practice of excision in Mali so as to enable them to reach a larger number of communities, especially the more isolated and remote communities that are found in the rural areas.

\section{To Centre Djoliba :}

- Share the lessons learned and Best Practices to support the process of elimination of excision;

- Strengthen the managerial capacity of the Centre Djoliba in order to optimize its human resources;

- Promote the creation of Support Clubs in all the intervention zones and involve the children and youth who are not enrolled in school;

- Continue the advocacy work within the coalition for a legal framework concerning the elimination of excision in Mali;

- Strengthen the dialogue with religious leaders;

- Systematize the documentation of the interventions and the communication between the different stakeholders at the community level;

- Strengthen the collaboration with decentralized government structures and other civil society organizations. 


\section{Introduction}

L'excision est une pratique séculaire que l'on rencontre surtout en Afrique. Ses motivations sont diverses et parmi elles, la préservation de la virginité, le contrôle de la sexualité de la femme, les croyances religieuses, etc. Elle est pratiquée aussi bien par les musulmans que les chrétiens et les animistes. On recense généralement trois formes d'excision pratiquées selon les régions et l'appartenance ethnique: l'ablation du clitoris (clitoridectomie), l'ablation du clitoris et d'une partie des petites lèvres (excision), l'ablation du clitoris, des grandes lèvres et des petites lèvres avec suture du sexe (infibulation).

\section{Situation de l'excision au Mali}

\section{a. Contexte}

Les données sur la pratique de l'excision fournies par les Enquêtes Démographiques et de Santé (EDS III et IV) effectuées en 2002 et en 2006 montrent que le Mali est l'un des pays où le taux de prévalence de l'excision est parmi les plus élevés. Malgré une baisse de cette prévalence, les données de l'EDS IV ont montré que l'excision reste une pratique très répandue au Mali puisque $85 \%$ des femmes ont déclaré avoir été excisées (la prévalence était de $92 \%$ en 2001).

La pratique de l'excision au Mali varie selon les ethnies et les régions. Elle est beaucoup plus importante dans les régions de Kayes (98,3\%), de Koulikoro $(97,4 \%)$, de Sikasso $(94,7 \%)$ et de Ségou $(92,2 \%)$, alors que dans la région de Mopti et de Tombouctou, elle est respectivement de $75 \%$ et de $44 \%$. Par contre dans les régions de Gao et de Kidal, l'excision ne concerne respectivement que $1,8 \%$ et de $0,9 \%$ des femmes.

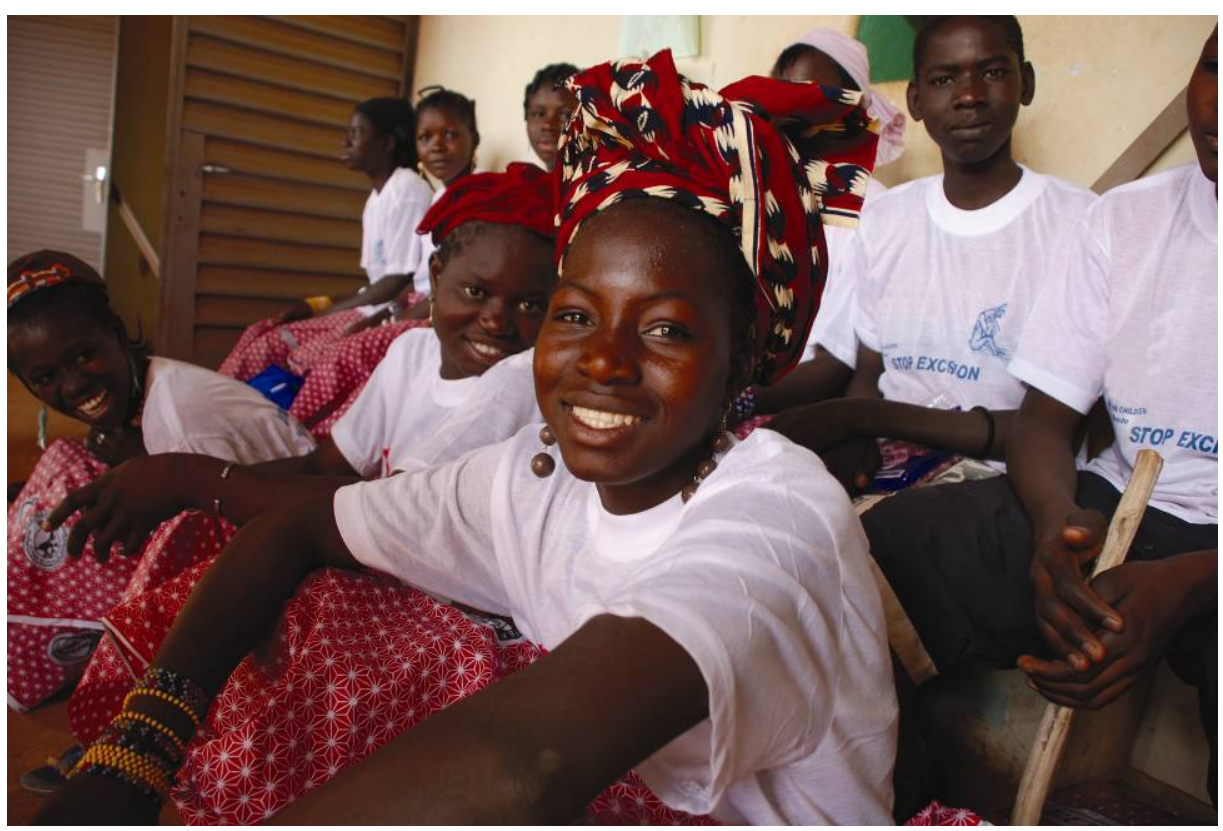

Les écarts se notent également selon les groupes ethniques. En effet, la pratique est très répandue parmi les Malinké (98\%), les Bambara $(97,7 \%)$, les Sarakolé/Soninké/Marka (96,9\%), les Peulh $(94,3 \%)$ et les Sénoufo/Minianka 
(92,9\%). Elle l'est moins chez les Bobos (81\%), les Dogon (76\%), les Tamacheks $(32 \%)$ et les Sonraï $(28 \%)$.

Les femmes animistes sont plus fréquemment excisées (92\%) que les femmes musulmanes $(86 \%)$ et les femmes chrétiennes (68\%).

La pratique touche toutes les couches sociales, des plus défavorisées aux plus aisées, même si elle semble être plus répandue parmi les femmes appartenant au quintile du bien-être économique le plus pauvre (93.7\%).

La majorité des femmes maliennes excisées $(60 \%)$ ont subi plus qu'une entaille, c'est-à-dire qu'une partie de leurs organes génitaux externes a été enlevée. Seulement 3\% des femmes excisées ont subi la forme légère de l'excision c'est-àdire une entaille. Paradoxalement, la pratique de l'infibulation semble avoir augmenté de moins de $2 \%$ en 2001 à $10 \%$ en 2006 , mais cela pourrait être dû à une formulation différente des questions de l'enquête.

Par contre, les résultats de l'EDS IV n'ont pas montré d'écart dans la pratique lié au niveau d'instruction.

L'excision reste une pratique traditionnelle au Mali dans la mesure où $92 \%$ des femmes excisées l'ont été par des praticiens traditionnels (89\% par les exciseuses traditionnelles et 3\% par des accoucheuses traditionnelles). Seules 3\% des femmes ont été excisées par des professionnels de santé. Elle est pratiquée très tôt dans la petite enfance (c'est-à-dire dans les jours et les mois qui suivent la naissance) dans $57 \%$ des cas.

Soixante neuf (69) pour cent des femmes interrogées avaient fait exciser leur fille et $14 \%$ d'entre elles avaient l'intention de le faire. Il est intéressant de noter que $12 \%$ des femmes non-excisées avaient déjà fait exciser leurs filles et que $2 \%$ d'entre elles en avaient l'intention. De façon générale, la pratique de l'excision au Mali se perpétue puisque seulement $4 \%$ des femmes non-excisées n'ont pas l'intention de faire exciser leurs filles.

Il est par ailleurs important de tenter de comprendre les raisons qui expliquent le maintien de la pratique de l'excision. Selon les hommes et les femmes interrogés lors de la dernière EDS, la pratique de l'excision présente plusieurs avantages tels que la reconnaissance sociale (37\%), l'obligation religieuse (24\%), une meilleure hygiène $(22 \%)$, la préservation de la virginité $(12 \%)$, l'assurance de meilleures chances de mariage (10\%) et le plaisir de l'homme dans $7 \%$ des cas.

\section{b. Cadre institutionnel des interventions en matière de lutte contre la pratique de l'excision au Mali}

Le Mali est engagé dans la lutte contre la pratique de l'excision depuis la fin des années 50 lors du Congrès des Femmes Africaines tenu à Bamako en 1959, rencontre durant laquelle la problématique de l'excision a été abordée. L'Union Nationale des Femmes du Mali (UNFM) prend l'initiative de mener le combat contre la pratique de l'excision et des ONG s'engagent à ses côtés dans cette lutte, les premières étant le Centre Djoliba et la Croix-Rouge.

En 1996, le Commissariat de la Promotion des Femmes met en place un Comité National d'Action pour l'Abandon des Pratiques Néfastes à la Santé de la Femme et de l'Enfant (CNAPN). Composé des représentants des secteurs ministériels, des institutions et des services techniques, des ONG et des partenaires au développement, ce comité est représenté au niveau régional par des CRAPN et dans les cercles par les CLAPN. 
L'engagement du gouvernement malien pour l'abandon de l'excision s'est traduit par plusieurs décisions dont :

- La signature de la lettre du 16 janvier 1999 du Ministère de la Santé interdisant l'excision en milieu médical ;

- L'adoption de la Loi du 24 juin 2002 sur la Santé de la Reproduction par l'Assemblée Nationale ;

- La création du Programme National de Lutte contre l'Excision (PNLE) en 2002 ;

- L'introduction dans le curriculum d'enseignement des établissements scolaires et socio-sanitaires d'un module de formation sur les problèmes posés par l'excision ;

- La tenue d'un forum national sur l'excision en décembre 2003 qui a permis d'aboutir à un consensus de tous les acteurs de la lutte ;

- L'adoption d'un plan d'action du PNLE pour 2003-2007;

- La célébration le 06 février de la journée "Tolérance zéro aux MGF" depuis 2004;

- La tenue d'un forum sous-régional sur le Protocole de Maputo.

Aujourd'hui près de 80 associations interviennent dans le cadre de la lutte contre la pratique de l'excision avec différents outils et stratégies tels que la formation, la sensibilisation et le plaidoyer.

Il est important de noter l'acceptation et l'implication de plus en plus forte de certains leaders religieux musulmans dans la lutte contre la pratique de l'excision.

Par ailleurs plusieurs réseaux ont été mis en place et s'activent pour l'abandon de cette pratique parmi lesquelles on peut citer :

- Le Réseau des Parlementaires maliens contre la pratique de l'excision;

- Le Réseau Malien des Parlementaires pour la Population et le Développement (REMAPOD);

- Le réseau des communicateurs traditionnels;

- Le réseau des communicateurs modernes (RMCAEX);

- Le réseau malien de lutte contre les MGF qui compte en son sein une cinquantaine d'ONG nationales de lutte contre l'excision.

Le PNLE est donc la structure nationale chargée de la mise en œuvre de la politique du Mali en matière de lutte contre l'excision, de la coordination, du suivi et de l'évaluation des actions de tous ces intervenants. Il appuie l'élaboration et la mise en œuvre des programmes sectoriels d'activités de lutte contre l'excision et celui des ONG et Associations.

Depuis 2002, le PNLE est un service rattaché au Ministère chargé de la Promotion de la Femme, de l'Enfant et de la Famille. Il a des points focaux au niveau des directions régionales du Ministère. En tant qu'organe de coordination, le PNLE est chargé entre autres tâches de coordonner toutes les activités de lutte contre la pratique de l'excision, de définir une stratégie d'information, d'éducation et de communication à l'endroit des membres de la communauté et des collectivités territoriales pour leur appropriation de la politique nationale de lutte contre la pratique de l'excision et de suivre et évaluer les activités des différents intervenants en matière de lutte contre l'excision. 
Depuis la création du PNLE, le Centre Djoliba collabore étroitement avec la structure, fait partie du CNAPN et mène des activités avec le PNLE en tant que participant mais aussi en tant que personnes ressources en ce qui concerne par exemple l'approche basée sur les droits de l'enfant. Le Centre Djoliba et le PNLE organisent conjointement des formations pour des institutions telles que les Ministères de l'Education, de la Communication, de la Justice et de la Santé sur le thème de l'excision.

\section{Justification de l'évaluation}

Save the Children Suède appuie donc les activités d'abandon de l'excision gérées par le Centre Djoliba au Mali depuis les années 80. Cependant, on observe un tournant en 2004. En effet, le "Projet de renforcement des capacités des groupes stratégiques pour l'abandon de l'excision et la promotion des droits des femmes et des enfants" financé par Save the Children Suède met le focus sur la dimension des droits humains. Le document du projet mentionne ce qui suit: "Le recul critique des différentes approches constitue un repère important et laisse entrevoir des insuffisances qu'il convient de combler.

Ainsi, à la recherche d'autres alternatives de lutte contre les pratiques néfastes et d'une diversification des messages de formation et d'information sur le sujet, le Centre Djoliba veut concevoir un projet sur le thème phare de la promotion des droits de la femme et de l'enfant." Avec cette nouvelle stratégie qui prend appui sur les droits humains, il s'agit de "placer la femme et l'enfant au centre du débat, tout en les considérant comme des acteurs sociaux" et de promouvoir la responsabilisation des communautés afin qu'elles trouvent une réponse locale à la pratique néfaste de l'excision.

Le projet est mis en œuvre dans six régions du Mali, à savoir Kayes, Koulikoro, Sikasso, Ségou, Mopti et le district de Bamako. Il consiste à soutenir financièrement et techniquement les activités de sensibilisation, de plaidoyer et de formation des groupes stratégiques, des animateurs relais du Centre Djoliba et des membres du Réseau malien de Lutte contre les Mutilations Génitales Féminines (RmL/MGF). Les groupes stratégiques concernés sont les autorités administratives, les élus locaux, les autorités villageoises, les agents de santé, les exciseuses, les responsables d'associations au niveau des zones de rayonnement du projet.

Le Centre Djoliba revendique les acquis suivants :

- Une plus grande implication des filles et garçons dans la lutte à travers la mise en place de clubs de soutien dans les communautés;

- L'abandon de la pratique par des exciseuses par conviction et leur implication dans les campagnes de sensibilisation et autres activités génératrices de revenus;

- L'investissement des enseignants acquis à la cause dans l'information des enfants;

- La mise en place de relais dans les communautés;

- L'abandon de la pratique par des villages;

- La formation des parlementaires désormais impliqués dans la lutte;

- Une plus grande implication des hommes; 
- L'existence de comités de vigilance dans les communautés qui ont abandonné la pratique;

- L'engagement de certains religieux qui prêchent contre l'excision;

- L'implication des radios communautaires.

Cependant, aucune évaluation systématique n'avait encore été menée pour démontrer l'impact de l'approche basée sur les droits de l'enfant adoptée par le Centre Djoliba depuis 2004. C'est dans cette optique que Save the Children Suède a envisagé de collaborer avec Population Council pour capitaliser cette expérience.

\section{Objectifs de l'évaluation}

L'objectif général de cette étude était d'évaluer l'efficacité et l'impact du programme du Centre Djoliba sur certains aspects de la vie des habitants des villages sélectionnés, par rapport à l'excision et notamment de :

- Démontrer l'impact des actions du Centre Djoliba avec le changement d'approche notamment l'approche basée sur les droits de l'enfant;

- Identifier les aspects à renforcer dans la mise en œuvre de l'approche basée sur les droits ;

- Capitaliser les expériences du Centre Djoliba dans le domaine de la lutte pour l'abandon de l'excision au Mali. 


\section{Méthodologie}

\section{Choix méthodologique}

Pour mettre en évidence l'impact des actions du Centre Djoliba sur le vécu des populations des zones où il est intervenu, le choix a été porté sur une étude qualitative en raison du court délai imparti à l'étude et du budget restreint alloué à ce travail d'évaluation. Cette étude qualitative a été précédée et accompagnée d'une étude documentaire sur la mobilisation contre l'excision au Mali de manière générale, et sur les actions du Centre Djoliba.

\section{Zones de l'enquête}

Pour avoir une représentation de la diversité ethnique et culturelle des régions où le Centre Djoliba a travaillé, quatre régions, à savoir Koulikoro, Sikasso, Ségou et Kayes, ont été choisies de façon raisonnée sur la base des informations fournies par le Centre Djoliba.

\section{Graphique I : Situation géographique des zones d'étude}

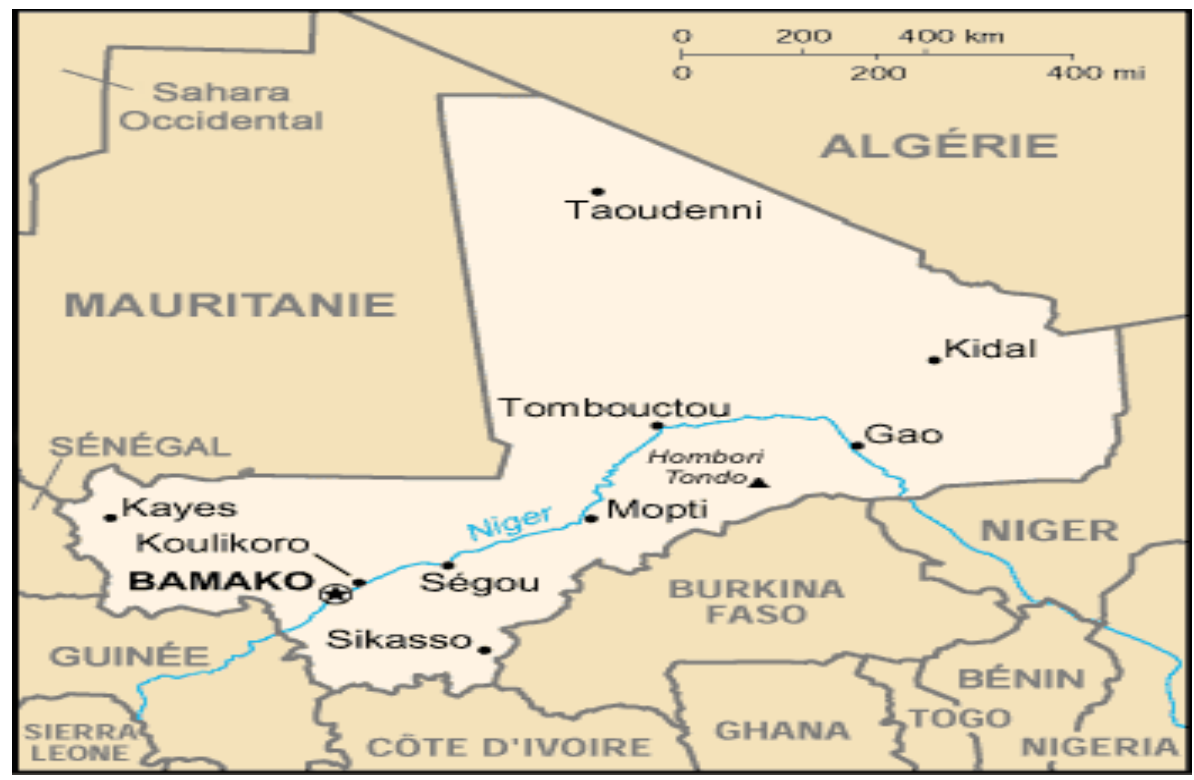

La population totale des sites concernés par l'étude est de 1.522.580 habitants. Le nombre de familles varie de 14 à 140 familles selon les villages. Les enfants de 5 à 14 ans représentent $28 \%$ de la population d'un village.

La composition ethnique dans les villages est: Bambara, Peulh, Sarakolé, Minianka, Bobo, Mossi, Dogon, Sénoufo, Bozo, Samoko, Malinké, Somono.

L'étude a été menée dans 12 villages et quartiers sélectionnés de façon raisonnée parmi les 153 sites où le Centre Djoliba mène ses interventions afin d'obtenir un échantillon représentatif de l'ensemble des zones d'intervention du Centre Djoliba. Ces 12 villages ont donc été retenus en tenant compte des spécificités de chacun d'eux et des critères ci-dessous : 
- villages ayant reçu l'intervention du Centre Djoliba ;

- abandon déclaré de l'excision par le village ;

- présence d'un comité de surveillance pour l'abandon de l'excision ;

- présence d'un club de soutien à l'abandon de l'excision ;

- engagement de la communauté de façon générale mais spécifiquement des femmes pour l'abandon de l'excision;

- présence de filles non-excisées ;

- résistance des communautés du village à l'abandon de l'excision.

Tableau I : Localités de l'enquête

\begin{tabular}{|c|c|c|}
\hline Région & Département/Cercle & Villages/Sites \\
\hline Koulikoro & Kati & $\begin{array}{l}\text { KATI SANANFARA : ASSSOCIATION } \\
\text { BENKADY } \\
\text { Kati Mission : ONG Tagne }\end{array}$ \\
\hline \multirow{3}{*}{ Ségou } & Ségou & $\begin{array}{l}\text { Ségou Ville : Antenne Centre Djoliba } \\
\text { Pelengana } \\
\text { Banankourou }\end{array}$ \\
\hline & Tominian & $\begin{array}{l}\text { Kossédougou } \\
\text { Sialo }\end{array}$ \\
\hline & San & Somo \\
\hline Sikasso & Yanfolila & $\begin{array}{l}\text { Yanfolila Ville } \\
\text { Gualala } \\
\text { Nousomna }\end{array}$ \\
\hline Kayes & Kita & $\begin{array}{l}\text { Karo } \\
\text { Katabantakoto }\end{array}$ \\
\hline
\end{tabular}

Les deux sites dans la région de Kayes ont été choisis pour servir de zones contrôles. En effet, contrairement aux autres villages sélectionnés, l'approche basée sur les droits n'a pas été mise en œuvre par le Centre Djoliba dans les villages de Karo et Katabantakoto, du fait que ceux-ci n'ont pas bénéficié de l'appui financier de Save the Children Suède.

\section{Méthodes et outils d’enquête}

Cette étude a mis en œuvre essentiellement deux méthodes classiques de la recherche socio-anthropologique que sont les entretiens individuels approfondis et les focus groupes, avec à l'occasion des entretiens informels. L'option prise a consisté à diversifier les catégories d'informateurs plutôt que les méthodes d'enquête. Ces méthodes ont été complétées par l'établissement des portraits de chaque localité d'enquête (description des principales caractéristiques et de la 
situation de chaque localité sur le plan économique, géographique, démographique, historique, culturel, sanitaire et autres).

La finalisation des outils d'enquête a pu se faire à la suite de la rencontre entre l'équipe d'évaluation de Population Council et le staff du Centre Djoliba. Trois outils de collecte ont ainsi été élaborés: les guides d'entretiens individuels approfondis, les guides d'entretiens de groupe (focus groupes) et les guides des portraits des villages.

\section{Les portraits des villages}

Dans les villages, le guide d'entretien pour l'établissement des portraits était adressé aux autorités villageoises. Il a permis d'avoir une bonne connaissance des caractéristiques et de la situation des villages choisis en relation avec l'excision. Ce guide a été administré uniquement par le superviseur de l'équipe avec la triangulation de l'information entre deux ou trois personnes informatrices. Les données statistiques au niveau des centres de santé et des mairies ont également été relevées par ses soins.

Les portraits des localités ont été parfois complétés par les entretiens avec des informateurs clés de certaines structures.

\section{Les focus groupes}

Les focus groupes ont eu lieu dans toutes les localités d'enquête (12). En fonction des localités, des catégories d'informateurs ont été ciblées pour participer aux groupes de discussion (hommes ou femmes adultes). C'est une méthode qui a pour but de permettre l'identification des opinions et tendances dominantes dans une communauté sur une question donnée, au-delà des opinions spécifiques de tel ou tel groupe selon le sexe, l'âge, le statut social, etc. Un guide d'entretien de groupe a été élaboré et les focus groupes ont tous été réalisés par le sociologue enquêteur. Quelques hommes et femmes ont ensuite été choisis parmi les participants aux focus groupes pour des entretiens individuels approfondis.

Comme mentionné plus haut, le démarrage des enquêtes proprement dit a été précédé d'une rencontre sous forme d'entretien de groupe, entre l'équipe d'évaluation de Population Council et tout le staff du Centre Djoliba. Cet entretien a non seulement permis de passer en revue la longue expérience du Centre Djoliba en matière de mobilisation contre l'abandon des pratiques néfastes, y compris l'excision, d'avoir des informations complémentaires sur l'approche droits, mais elle a aussi aidé dans le choix des villages à retenir pour l'évaluation et à affiner les différents outils de collecte.

\section{Les entretiens individuels approfondis}

Les guides ont été élaborés pour collecter des données sur la pratique de l'excision, les perceptions et attitudes des populations, la connaissance du mouvement de lutte contre l'excision à travers le Centre Djoliba, les perspectives pour le changement de comportement des communautés notamment l'abandon de l'excision. La notion d'approche basée sur les droits de l'enfant, son application et les effets voire l'impact des activités du Centre Djoliba ont aussi été explorés.

Les entretiens individuels ont eu pour cible aussi bien le niveau central que les différentes catégories d'informateurs dans les communautés. 


\section{Echantillonnage}

Quatre unités d'échantillonnage ont été retenues:

- Le Centre Djoliba ;

- Les intervenants/partenaires du Centre Djoliba dans la lutte contre l'excision ;

- Les facilitateurs/relais et autres agents que le Centre Djoliba utilise ;

- La communauté bénéficiaire ;

Au niveau central, les structures suivantes ont été interviewées :

- La Division de la Santé de la Reproduction (Ministère de la Santé);

- Le Programme National de Lutte contre l'Excision (PNLE);

- Un député de l'Assemblée Nationale (membre du Réseau des Parlementaires Maliens);

A Bamako ville, un relais du Centre Djoliba, responsable d'un centre de formation en couture dans un quartier périphérique de Bamako ainsi que des jeunes femmes bénéficiaires de ce centre ont été interrogés.

Au niveau de chaque localité, l'enquête a ciblé les catégories d'informateurs suivantes :

- Leaders hommes âgés;

- Leaders hommes jeunes;

- Leaders religieux;

- Leaders femmes;

- Femmes participantes aux activités du Centre Djoliba;

- Femmes non participantes aux activités du Centre Djoliba;

- Filles participantes aux activités du Centre Djoliba;

- Filles non participantes aux activités du Centre Djoliba;

- Filles non-excisées;

- Exciseuses non pratiquantes;

- Exciseuses pratiquantes;

- Leaders du comité de surveillance;

- Membres du comité de surveillance;

- Leaders du club de soutien;

- Membres du club de soutien;

- Relais du Centre Djoliba;

- Autres personnes spécifiques selon les villages: le directeur du Centre d'Animation Pédagogique (CAP), le directeur de la radio; le médecin chef...

Les guides d'entretiens individuels ont été adaptés selon la catégorie d’informateurs ciblée en ne retenant que les questions pertinentes.

L'étude a touché près de 200 personnes au total. 
Tableau 2 : Nombre de focus groupes, d'entretiens individuels et de portraits de villages réalisés

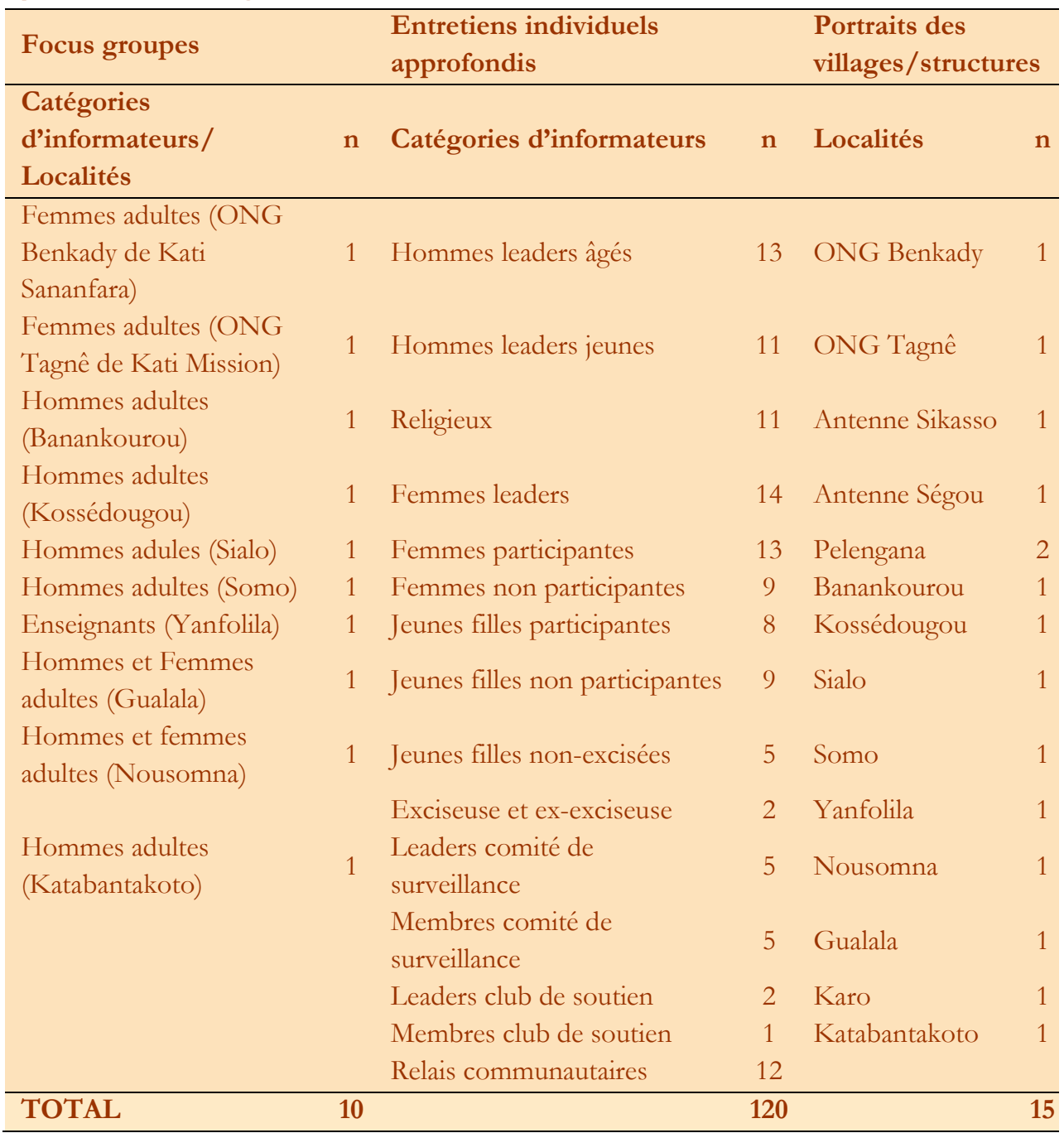

\section{Déroulement de l'enquête}

La formation des membres de l'équipe de recherche par deux responsables de Population Council s'est déroulée pendant quatre jours ; l'équipe pluridisciplinaire de recherche était composée de cinq personnes (un sociologue, trois médecins spécialistes en santé communautaire et une technicienne de santé spécialiste en santé publique) ayant une expérience avérée dans la collecte de données qualitatives et une bonne connaissance de la problématique de l'excision et des villages couverts par l'étude.

Une journée a été consacrée au test des outils dans le village de Bémasso Kabarou situé à environ $20 \mathrm{~km}$ du cercle de Kati (commune de Kambila). Ce pré-test a permis de vérifier la clarté et la pertinence des questions adressées aux différentes cibles de l'évaluation, mais aussi de s'assurer de la qualité de la traduction des questions en langue Bambara.

La collecte des données quant à elle s'est déroulée sur un mois. L'équipe de recherche a été scindée en deux: une a été chargée de mener les enquêtes au niveau des services centraux dans le District de Bamako, ainsi qu'une revue 
documentaire sur le phénomène de l'excision et les interventions de lutte contre cette pratique au Mali; la deuxième équipe s'est chargée de la collecte des données dans les localités d'enquête. Les interviews des responsables du PNLPE, du Ministère de la santé et du Réseau des Parlementaires Maliens ont été menées par les chercheurs de Population Council.

L'équipe de recherche a rencontré certaines difficultés liées pour la plupart à l'éloignement de certains sites. Par ailleurs, les informateurs étant pris dans leurs activités quotidiennes, les enquêteurs ont parfois dû attendre dans un site que ces derniers soient disponibles pour répondre à leurs questions. Dans certains cas, la barrière de la langue a nécessité le recours à un traducteur, ce qui entraîne des risques de perte de qualité de l'information. 


\section{I- Présentation du Centre Djoliba}

Créé le 05 Janvier 1964, le Centre Djoliba se veut une structure d'appui pour le renforcement des capacités d'intervention des acteurs et actrices du développement. Disposant d'une équipe de 22 personnes dont 8 formateurs professionnels, 5 spécialistes en bibliothéconomie et gestion documentaire et des personnes ressources (économistes, sociologues, juristes, médecins, communicateurs, ingénieurs...), il mène des activités pour la promotion du développement sous toutes ses formes à travers des sensibilisations, des formations, un centre de documentation et des activités d'appui aux initiatives de développement (groupements de femmes, de jeunes, etc.) et lutte contre les pratiques traditionnelles néfastes. Le centre dispose de deux antennes régionales à Sikasso (2002) et à Ségou (2004) et intervient auprès des ONG, des groupements de femmes et de jeunes, des communautés villageoises et des collectivités territoriales.

\section{Vision et mission du Centre Djoliba}

Dans les années 1990, le Centre Djoliba a réellement commencé à dérouler des programmes spécifiquement orientés vers la lutte contre la pratique de l'excision au Mali. Depuis cette date, l'ONG malienne a une vision de l'excision en tant que pratique qui interpelle à la fois les dimensions anthropologique, ethnologique, religieuse, sanitaire et celle du droit humain. Pour cela, le Centre Djoliba s'est donné les missions suivantes :

- Créer le débat et un dialogue sur la pratique de l'excision et ses conséquences sur les femmes et les filles;

- Harmoniser les approches des différents intervenants sur le terrain en rendant disponible une documentation et des outils pédagogiques et en renforçant leurs capacités ;

- Sensibiliser les différents acteurs sociaux à travers des actions d'information comme des émissions radio et télé, le théâtre;

- Apporter aux décideurs et aux partenaires au développement des éléments qui leur permettent d'éclairer et de réorienter leurs recherches et leurs actions ;

- Promouvoir les droits de la femme et de l'enfant à la santé, à l'intégrité physique;

- Apporter aux femmes et aux filles victimes d'excision un soutien et un accompagnement sous forme de conseils et d'orientation;

- Amener les exciseuses à se reconvertir dans d'autres créneaux porteurs. 


\section{Programme d'activités}

Le Centre Djoliba a deux départements principaux :

- Le département Documentation et Débats Publics.

- Le département Formation qui comprend :

- Le volet Formation ;

- Le volet Insertion sociopolitique de la femme ;

- Le volet Promotion de la Santé de la Reproduction

Dans le cadre du volet "promotion de la Santé de la Reproduction », le Projet d'Education pour l'abandon de l'excision et la promotion des droits de la femme et de l'enfant a été mis en œuvre en mettant en exergue la nouvelle orientation du Centre Djoliba qui va de l'approche Santé à l'approche basée sur les droits. Les étapes suivantes peuvent être observées dans la mise en œuvre des activités du Centre Djoliba en matière de lutte pour l'abandon de la pratique de l'excision :

1983 - 1999 : Le Centre Djoliba a utilisé au cours de cette période l'approche axée sur la santé qui consistait à se focaliser uniquement sur les conséquences sanitaires de l'excision. L'excision était alors considérée principalement comme une question de femmes. Les activités se limitaient à la sensibilisation de groupements et associations de femmes.

1999 - 2003 : Suite à une évaluation commanditée par Save the Children Suède vers la fin de l'année 1999, le Centre Djoliba a revu sa stratégie pour impliquer certains groupes stratégiques, en particulier les hommes et les jeunes. Cependant, l'approche était toujours axée sur la santé des femmes et le message principal tournait autour des conséquences néfastes de l'excision sur la santé de ces dernières.

2004-2008 : Suite au constat que l'approche santé entrainait une médicalisation de l'excision, le Centre Djoliba adopte l'approche basée sur les droits de l'enfant prônée par Save the Children Suède. La question de l'excision est désormais considérée comme une violation des droits de l'enfant, en particulier de la petite fille et de la femme. Cette approche se base sur la Convention des Nations Unies relative aux Droits de l'Enfant, la Charte Africaine des Droits et du Bien-être de l'enfant et la Convention pour l'Elimination de toute forme de discrimination à l'égard des femmes, ainsi que tous autres textes juridiques au niveau national pour amener les populations à assurer le bien-être des enfants.

Désormais la question de l'excision n'est plus traitée de manière isolée mais elle fait partie d'un programme holistique qui traite de tous les problèmes auxquels les enfants sont confrontés. Progressivement, les populations sont amenées à changer de mentalité et à abandonner la pratique de l'excision de leur plein gré.

D'après l'approche basée sur les droits, l'Etat est considéré comme le premier responsable pour la réalisation des droits de l'enfant d'où l'importance du travail de plaidoyer au niveau national.

Tous les acteurs à savoir les parents, ainsi que tous les autres membres de la communauté sont également ciblés comme étant responsables de la mise en application des droits de l'enfant. Dans l'approche basée sur les droits, les enfants et les femmes sont considérés comme des acteurs qui peuvent également contribuer à la lutte contre l'excision et non seulement des victimes. 
"Avec cette approche, nous remarquons que la plupart des enfants sont bien éduqués et bien entretenus par leurs parents. Par rapport à certaines questions les concernant, les jeunes sont impliqués ou consultés dans la prise de certaines décisions.» (Femme leader de Banankourou)

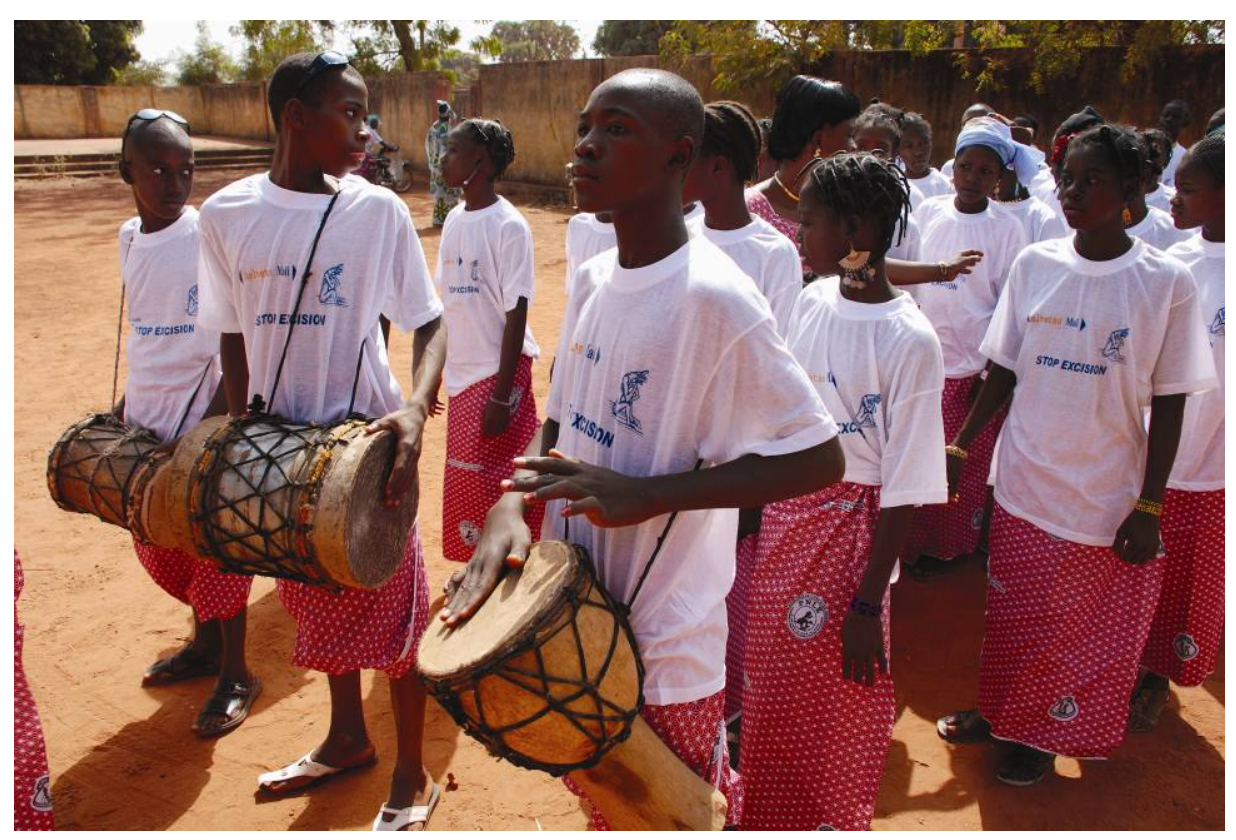




\section{II- Processus d'intervention du Centre Djoliba}

\section{Stratégie de mise en œuvre du programme par le Centre Djoliba}

\section{a. Renforcement des capacités}

- Formation des relais

Afin de toucher la majorité de la population, le Centre Djoliba s'appuie sur les relais au niveau communautaire. Il dispose d'un noyau de 52 relais composés de femmes et d'hommes. Ces relais ont été formés sur l'approche basée sur les droits de l'enfant et bénéficient de formations continues sur les techniques de communication, de collecte de données sur le terrain et autres thématiques liées au bon déroulement de leur travail. Ces relais ainsi formés retournent dans les villages d'intervention du Centre Djoliba afin d'organiser des séances de sensibilisation dans les communautés y compris les écoles et les lycées. Cette sensibilisation se fait à travers des causeries avec la méthode GRAAP, des pièces de théâtres, des émissions radiophoniques sur la communication pour un changement de comportement, la santé de la reproduction, l'excision, les droits de l'enfant, entre autres. Depuis 2003, l'approche basée sur les droits est utilisée de façon systématique.

\author{
Le Centre Djoliba a mis en \\ œuvre d'autres activités \\ pour la reconversion des \\ exciseuses en assurant \\ (...) l'accompagnement \\ des femmes en \\ alphabétisation et en \\ gestion de microcrédits.
}

- Formation des groupes stratégiques.

Dans l'approche basée sur les droits, la question de l'excision est considérée comme un problème communautaire. C'est dans cette optique que le Centre Djoliba forme les groupes stratégiques qui sont incontournables dans le processus d'abandon de la pratique de l'excision tant au niveau des communautés qu'au niveau national. Il s'agit des autorités communales et villageoises, les leaders religieux et d'opinion, les communicateurs traditionnels et modernes, les enseignants, les groupements et associations de femmes et de jeunes, les agents de santé, les exciseuses, les parlementaires et les responsables au niveau de certaines institutions étatiques. Une fois que ces différents groupes stratégiques sont formés, ils constituent des personnes ressources ou deviennent des relais qui jouent un rôle important dans la sensibilisation et le plaidoyer au sein de leurs communautés et au niveau national.

- Encadrement des femmes

Plusieurs associations féminines ont bénéficié de l'encadrement du Centre Djoliba qui, dans certains cas, a été à l'origine de la création de ces associations ou a aidé à redynamiser celles qui existaient déjà. Les femmes membres de ces groupements ont ainsi bénéficié d'un renforcement de capacités dans les domaines de la santé de la reproduction (excision, IST/SIDA, PF), de la santé maternelle et infantile, des droits de l'enfant, de l'hygiène et de l'assainissement, avec notamment l'objectif de faire d'elles des agents de sensibilisation des autres membres de la communauté.

Le Centre Djoliba a mis en œuvre d'autres activités pour accompagner la reconversion des exciseuses en assurant la formation des matrones et 
Les clubs de soutien regroupent 20 à 250 enfants et jeunes. Ils ont surtout été créés pour former et encadrer les enfants, mais les activités des enfants débordent le cadre strict de cette mission puisqu'ils mènent également des activités de sensibilisation et de plaidoyer auprès des membres de leurs communautés.
Les autres acteurs institutionnels présents dans le champ de la mobilisation contre l'excision ont été intéressés par l'approche basée sur les droits. accoucheuses traditionnelles et l'accompagnement des femmes en alphabétisation et en gestion de microcrédits. En effet, à la demande des femmes et avec l'appui d'autres partenaires et avec ses fonds propres, le Centre Djoliba a aidé les femmes à s'organiser et renforcer leurs capacités de gérer leurs propres activités génératrices de revenus.

\section{- Participation des enfants}

A l'initiative des enseignants formés par le Centre Djoliba, des clubs de soutien vont être créés dans certaines écoles et lycées regroupant des élèves qui seront à leur tour sensibilisés et formés sur la problématique de l'excision, les droits de l'enfant et les techniques de sensibilisation, afin de devenir des pleins acteurs de la lutte pour l'abandon de la pratique de l'excision.

Au nombre de 13 au total, les clubs de soutien regroupent 20 à 250 enfants et jeunes. Ils ont surtout été créés pour former et encadrer les enfants, mais les activités des enfants débordent le cadre strict de cette mission puisqu'ils mènent également des activités de sensibilisation et de plaidoyer auprès des membres de leurs communautés.

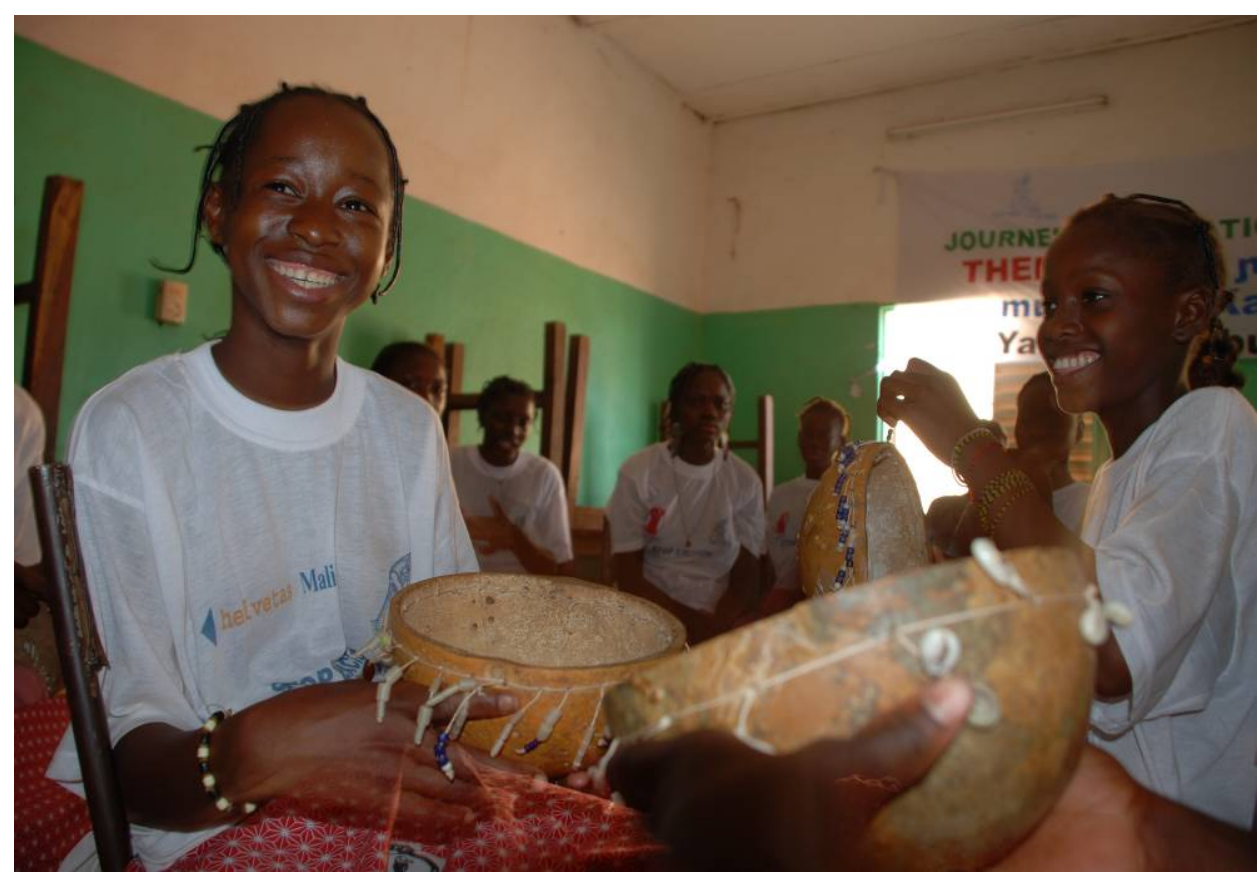

- Renforcement des capacités du PNLE et transfert de compétence

En ce qui concerne le PNLE, la collaboration avec le Centre Djoliba se traduit surtout en termes de renforcement de capacités et de transfert de compétences. En effet, il apparaît que l'approche santé avait ouvert beaucoup de portes, mais elle avait aussi montré ses limites dont l'une des plus importantes est la médicalisation de l'excision qui va se pratiquer en milieu médical pour en minimiser les conséquences néfastes sur la santé des femmes.

Les autres acteurs institutionnels présents dans le champ de la mobilisation contre l'excision ont été intéressés par l'approche basée sur les droits mais avec l'appréhension d'être rejetés car non compris par les populations. Le PNLE a donc approché le Centre Djoliba afin de mieux comprendre comment introduire cette approche. 
Convaincu que par des notions simples, tirées des besoins de la vie quotidienne, on peut arriver à démontrer que les enfants aussi ont des droits fondamentaux, le PNLE a décidé de proposer la mise en œuvre de l'approche droits aux autres ONG et associations qu'il encadre et a sollicité le Centre Djoliba pour la formation de ses agents sur cette nouvelle approche.

Par ailleurs le Centre Djoliba a appuyé le PNLE dans la formation des ministères de l'éducation, de la communication, de la justice et de la santé sur l'excision. Aujourd'hui, par cette transmission de l'expertise, beaucoup de partenaires sont en train de passer à l'approche basée sur les droits. Par ailleurs, avec le financement de l'UNICEF, le PNLE a révisé son manuel de formation en y intégrant cette approche. C'est cette collaboration étroite entre le PNLE et le Centre Djoliba qui a fait dire que :

"Le Centre Djoliba est l'association pionnière de cette lutte contre l'excision au Mali. Il a beaucoup fait pour le PNLE et il constitue une structure de référence pour nous. (...) Le Centre Djoliba nous a fait beaucoup de formations qui nous ont permis de pouvoir mener des séances de sensibilisation sur le terrain et d'acquérir les changements de comportements des communautés vis-à-vis de l'excision."(Directrice du PNLE).

\section{b. Plaidoyer}

- Pour un cadre juridique de l'abandon de la pratique de l'excision

Un aspect important de l'approche basée sur les droits est le plaidoyer en faveur d'un cadre juridique au niveau national pour la protection des enfants. C'est à cet effet que le Centre Djoliba milite au sein des réseaux et coalitions suivants pour amener l'Etat du Mali à adopter une loi pour l'abolition de la pratique de l'excision: Réseau Malien de lutte contre les mutilations génitales féminines; Plateforme d'action, de plaidoyer et de lobbying contre les inégalités sociales (PAP); Consortium WILDAF- HELVETAS Centre Djoliba; Pacte pour la loi.

Le Centre Djoliba a également collaboré avec le Réseau des parlementaires maliens pour la population et le développement pour renforcer le plaidoyer en faveur d'une loi contre la pratique de l'excision au Mali. En effet, depuis la Conférence Internationale du Caire (1994), les parlementaires se sont engagés dans un plaidoyer pour un cadre juridique pour l'abandon de la pratique de l'excision au Mali. Dans ce cadre, le Réseau des Parlementaires Maliens pour la Population et le Développement a entamé une collaboration avec le Centre Djoliba, le réseau sénégalais et le forum pour les questions de populations et de développement. Le Centre Djoliba a donc formé les parlementaires sur l'excision et les droits de l'enfant pour assurer une bonne compréhension des enjeux de la problématique.

Par ailleurs, les membres du REMAPOD ont entrepris des actions de sensibilisation au sein de leurs circonscriptions.

- Pour l'implication des structures étatiques locales dans la lutte contre la pratique

Au niveau local, l'excision commence à être graduellement intégrée dans les Plans de Développement Social, Economique et Culturel (PDSEC) de certaines communes après la sensibilisation et la formation des élus locaux et des populations par le Centre Djoliba. En effet, le PDSEC sert d'outil de planification quinquennal et d'orientation de la collectivité décentralisée et prend en charge les activités de développement de la commune. Il est assorti d'un budget d'évaluation des activités programmées. L'objectif général du PDSEC est d'améliorer les
Le Centre Djoliba a appuyé le PNLE dans la formation des ministères (...) sur l'excision. Aujourd'hui, par cette transmission de l'expertise, beaucoup de partenaires sont en train de passer à l'approche basée sur les droits. 
"Avant chaque

animation, nous

informons d'abord le

chef du village et les

leaders des femmes,

jeunes et des hommes

du village. Après, le

crieur public passe dans

tout le village pour faire

l'annonce. Souvent,

l'annonce est faite

également à la radio

locale pour inviter tout

le village sur la place

publique." (Relais,

Kossédougou). conditions de vie des populations de la Commune. Il prend en compte tous les domaines de développement.

"L'excision constitue un volet dans la santé publique et est prise en compte dans le PDSEC. Aussi, mêmes les agents de santé luttent contre la pratique. » (Relais de Kossédongou).

\section{c. Suivi des activités}

Le suivi des activités sur le terrain se fait grâce à des outils tels que les fiches d'animation qui sont ramenées au Centre Djoliba, mais aussi des fiches de suivi pour avoir des informations sur les personnes formées. Des missions de supervision sont menées sur le terrain de façon trimestrielle. Une mission par zone est prévue. Les données sont recueillies tous les trois mois. Un rapport annuel est élaboré et les fiches d'animation sont réalisées pour chaque activité.

Ce travail de suivi est effectué par un seul agent du Centre Djoliba alors que le programme d'intervention couvre 153 villages et quartiers. Il en résulte un problème de coordination et de communication mais surtout une insuffisance de suivi et de capitalisation de l'ensemble des acquis obtenus sur le terrain, d'autant plus que les outils de suivi utilisés ne sont pas tout à fait adéquats.

\section{Stratégie de mise en œuvre du programme dans les communautés}

\section{a. Processus d'introduction du programme}

L'introduction du programme d'intervention dans les sites s'est faite par étapes. En effet, les activités du Centre Djoliba dans un village commencent par une enquête du milieu suivie de réunions d'information avec les autorités et les représentants de l'Etat. Puis des rencontres ont lieu avec les hommes, les femmes et les jeunes sur le contenu et les objectifs du programme d'intervention afin d'édifier les communautés sur les enjeux de leur participation. Ces rencontres sont organisées avec l'appui des relais ou ONG partenaires au niveau local.

A noter que le Centre Djoliba a délibérément choisi cette approche lente mais progressive pour ne pas choquer les mentalités et les habitudes. La présentation claire des objectifs du programme dès les premiers contacts a permis aux communautés de bien appréhender les enjeux de leur participation à ce programme et en particulier ceux de l'abandon de l'excision.

"Des concertations ont eu lieu pour déterminer les avantages et les inconvénients pour le village. Après des concertations dans le village, nous avons accepté de collaborer avec le Centre Djoliba. Ils sont venus nous expliquer leurs activités. Après, nous avons fait des concertations dans le village pour avoir le consensus de tout le monde." (Chef de village Katabantakoto)

Les difficultés principales viennent des résistances des groupes des religieux musulmans, de certaines exciseuses et de leurs apprentis. Si, pour certains villages, l'accord pour participer au programme a été obtenu facilement, pour d'autres il y a eu beaucoup de réticences, notamment de la part des groupes précédemment cités. 


\section{b. Déroulement du programme}

- Les acteurs impliqués

Différentes catégories d'acteurs ont été impliquées par le Centre Djoliba. A l'analyse, les approches privilégiées sont notamment la formation des formateurs (relais communautaires, ONG locales partenaires et autres) sur les thèmes du programme et la formation des leaders et membres des groupements et associations des différentes catégories sociales de la communauté (jeunes, élèves, femmes, leaders traditionnels et religieux, leaders politiques, ex-exciseuses).

L'ensemble de ces acteurs interviennent dans la sensibilisation et le plaidoyer. En plus de ces activités, les relais assurent la formation. Les personnes ainsi formées sont chargées de diffuser les informations auprès des communautés à travers la Communication pour le Changement de Comportement.

- Les activités

Les acteurs impliqués se sont surtout déployés dans les activités de sensibilisation et de formation suivantes :

La sensibilisation des pairs sur des thèmes portant sur l'excision et les droits de l'enfant ainsi que beaucoup d'autres sujets tels que la santé de la reproduction (SR), la santé de l'enfant, la lutte contre les IST et le VIH/SIDA, le paludisme, l'hygiène du milieu, le mariage précoce, etc. De manière générale, il apparaît que les personnes interrogées pensent que les droits de l'enfant sont un thème novateur par rapport à ceux précédemment abordés avec le Centre Djoliba, ce qui a facilité la tenue des séances d'IEC sur l'excision.

"Au départ, les relais nous parlaient de l'adolescence de l'enfant à 15 ans, la petite fille commence à être femme et le petit garçon un homme; le cycle menstruel, les périodes de fécondité, les rapports sexuels, l'assiduité à la CPN et l'accouchement au dispensaire, l'excision et ses complications pour la vie de la fille. Ils sont venus nous apporter des affiches avec les complications de l'excision. Enfin ils nous parlaient des droits des enfants." (Femme participante, Kossédougou).

Les lieux de mobilisation des communautés sont variés et sont généralement les lieux habituels de regroupement : il s'agit des écoles, des grins ${ }^{1}$, des lieux de culte, etc. Il est généralement fait appel au crieur public et à la radio locale pour mobiliser la population.

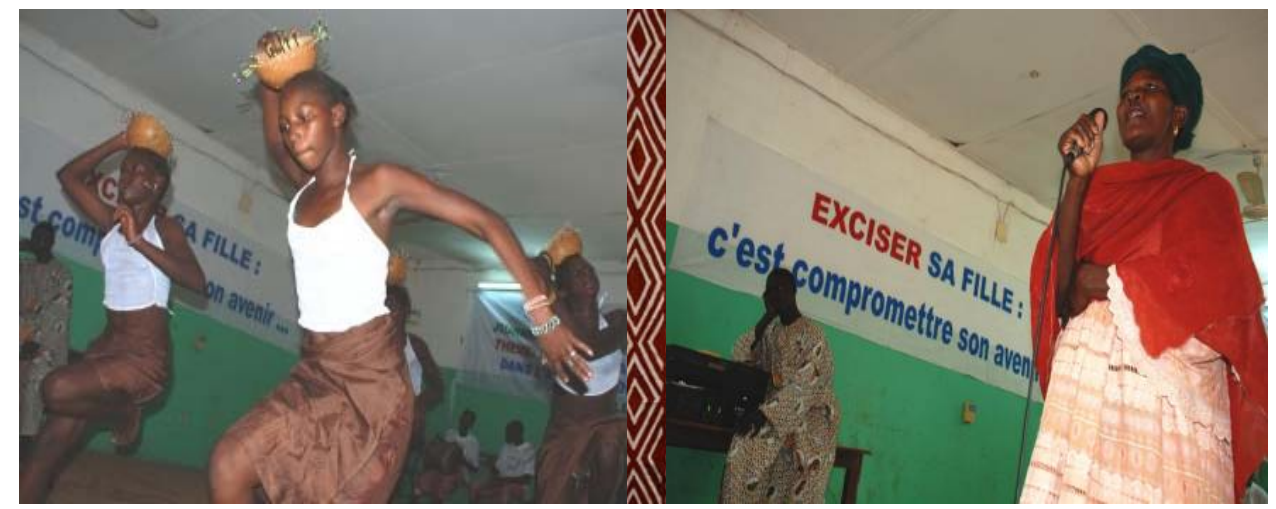

\footnotetext{
"Les interventions du Centre Djoliba sont bien écoutées par la population car même ceux qui n'y participent pas en savent quelque chose à travers les échos des autres." (Femme non participante Kati, Sananfara).
}

\footnotetext{
${ }^{1}$ Lieu fixe de rencontre, espace de socialisation typique des sociétés urbaines maliennes; lieu des échanges pour des personnes qui sont de la même classe d'âge
} 
"Avant chaque animation, nous informons d'abord le chef du village et les leaders des femmes, jeunes et des hommes du village. Après, le crieur public passe dans tout le village pour faire l'annonce. Souvent, l'annonce est faite également à la radio locale pour inviter tout le village sur la place publique." (Relais, Kossédougou).

Les moyens et outils de communication généralement utilisés par les différents acteurs sont la radio, les outils IEC, le théâtre et la communication interpersonnelle.

"J'ai reçu pas mal d'informations à travers les causeries entre nous, les femmes, au marché ou aux champs." (Femme non participante, Kossédougou).

On peut affirmer que la circulation de l'information est réelle et effective dans la mesure où presque tous les informateurs, y compris les femmes et les jeunes filles non participantes ont dit avoir eu des informations sur les thèmes développés avec l'intervention du Centre Djoliba.

"Les interventions du Centre Djoliba sont bien écoutées par la population car même ceux qui n'y participent pas en savent quelque chose à travers les échos des autres." (Femme non participante Kati, Sananfara).

Cependant, la qualité de l'information reçue varie selon que la personne a participé ou pas à une formation ou une activité de sensibilisation. Par ailleurs, le thème de l'excision (et ses effets néfastes pour la santé) est mieux connu de ceux qui ont participé aux activités.

Le lobbying mené par les associations de jeunes auprès des personnes âgées et des leaders religieux en mettant l'accent sur les actions planifiées a permis d' obtenir leur adhésion au programme. Par ailleurs, les femmes entre elles organisent des séances de sensibilisation pour augmenter le taux d'adhésion des femmes.

Les formations des acteurs sur le terrain sont assurées par les relais ayan été euxmêmes formés par le Centre Djoliba.

\section{c. L'adhésion au programme et à ses messages}

Après une première étape de réticence voire même d'hostilité observée au début, la majorité des communautés ont adhéré aux activités du programme.

Cette adhésion se manifeste par le nombre de personnes qui assistent aux séances d'IEC, les nouvelles adhésions dans les groupements et associations de femmes et la sollicitation des relais pour l'animation d'autres séances d'IEC.

"La comprébension est bonne car maintenant personne ne nous insulte en nous disant "est-ce que vous-mêmes vous n'êtes pas excisée ?". Maintenant nous sommes beaucoup sollicitées par la population." (Relais, Somo).

"L'intervention du Centre Djoliba intéresse bien les villageois au vu du nombre de participants aux différentes activités et cela à cause des informations relatives aux inconvénients de l'excision." (Jeune homme leader, Somo).

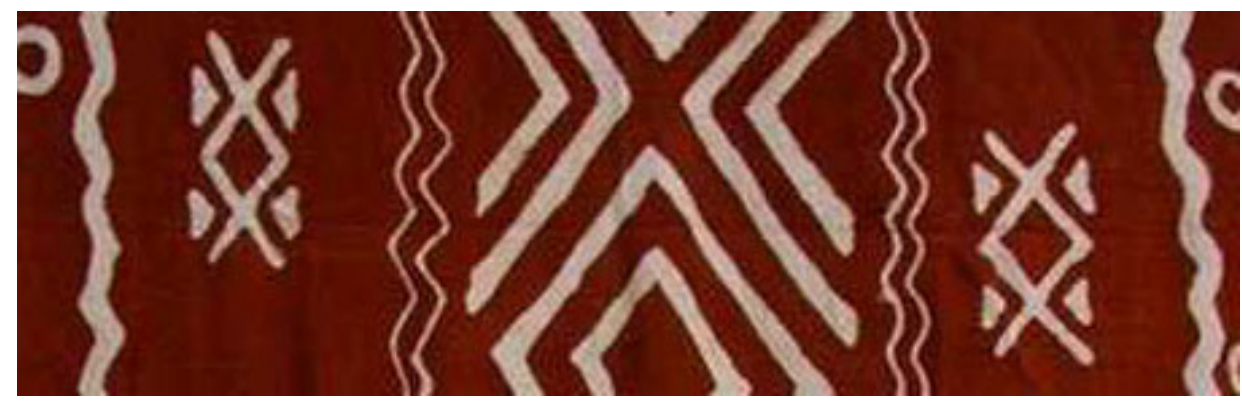




\section{III- Résultats de l'évaluation}

Un des impacts les plus importants de l'intervention du Centre Djoliba dans les communautés a été l'abandon ou la régression de la pratique de l'excision. En effet, l'abandon de l'excision est un des éléments les plus cités par les informateurs comme changement intervenu suite au déroulement du programme du Centre Djoliba. C'est le résultat d'un long processus de questionnements et de remise en cause de la pratique ancestrale de l'excision qui a mené vers sa régression ou son abandon.

\section{Evolution des connaissances, opinions et attitudes}

\section{a. L'excision n'est plus un sujet tabou}

Plusieurs informateurs, notamment les relais communautaires qui sont en quelque sorte à l'avant-garde de la mobilisation, ont mis en évidence le fait que le sujet de l'excision, précisément celui de son abandon par les communautés, est actuellement abordé de manière plus ouverte dans les villages. Ils ne manquent pas de mettre ce changement à l'actif des activités que le Centre Djoliba a déroulées. En effet, avant l'intervention du Centre Djoliba, il était difficile d'aborder le sujet de l'excision mais encore plus celui de son abandon. Dans beaucoup de ces villages, les séances d'animation n'étaient presque pas suivies et souvent les animateurs étaient victimes de violence verbale.

"Au départ des activités, les gens nous traitaient de n'importe quoi et parfois, ily en a même qui nous insultaient. Mais aujourd'bui, nous sommes plus écoutées et on constate bien que les gens commencent à comprendre l'approche." (Relais, Kossedougou).

Pour certains villages, tel celui de Pelengana, la levée du tabou est encore plus manifeste car certains leaders, musulmans notamment, se sont investis dans la sensibilisation contre l'excision et le sujet fait même l'objet de prêches dans les mosquées :

"Auparavant, on ne pouvait pas parler de ce sujet, mais aujourd'bui on en parle et les populations commencent à prendre conscience. On ne pouvait pas aborder le sujet dans les mosquées mais on le fait aujourd'bui car même le Coran ne parle pas d'excision. Ce n'est donc pas une obligation musulmane." (Chef de village, Pelengana).

La levée du tabou qui frappait le sujet de l'excision est un aspect non négligeable des changements intervenus car auparavant, des populations se sentaient offensées par des émissions radiophoniques de sensibilisation sur l'excision, et avaient organisé des manifestations pour demander leur arrêt.

\section{b. Meilleure connaissance des effets néfastes de l'excision}

Grâce aux interventions menées dans les villages par les relais communautaires et les différents groupes stratégiques ou la sensibilisation à travers les radios, les populations ont accès à une masse relativement importante d'informations sur différents aspects concernant l'excision, y compris, ses effets néfastes. Ainsi, beaucoup d'informateurs ont énuméré une litanie d'inconvénients que sont les complications et les conséquences négatives de l'excision au plan médical et psychosocial chez la petite fille, la femme et la mère. Les effets néfastes qui reviennent le plus souvent sont l'hémorragie, les risques de contamination des IST et du SIDA, les difficultés pour uriner ou l'incontinence urinaire, les
"Auparavant, on ne pouvait pas parler de ce sujet, mais aujourd'hui on en parle et les populations commencent à prendre conscience. On ne pouvait pas aborder le sujet dans les mosquées mais on le fait aujourd'hui car même le Coran ne parle pas d'excision. Ce n'est donc pas une obligation musulmane." (Chef de village, Pelengana). 
difficultés liées à l'accouchement, la stérilité, la perte de la libido chez la femme et donc son incapacité à satisfaire sexuellement son partenaire entraînant des conflits et des difficultés dans le couple, et enfin la mort.

\section{c. Mutations dans la réflexion et les attitudes}

L'accès des populations à une information plus complète et exhaustive sur la pratique coutumière de l'excision a eu comme effet une mutation profonde au niveau de la réflexion et des attitudes.

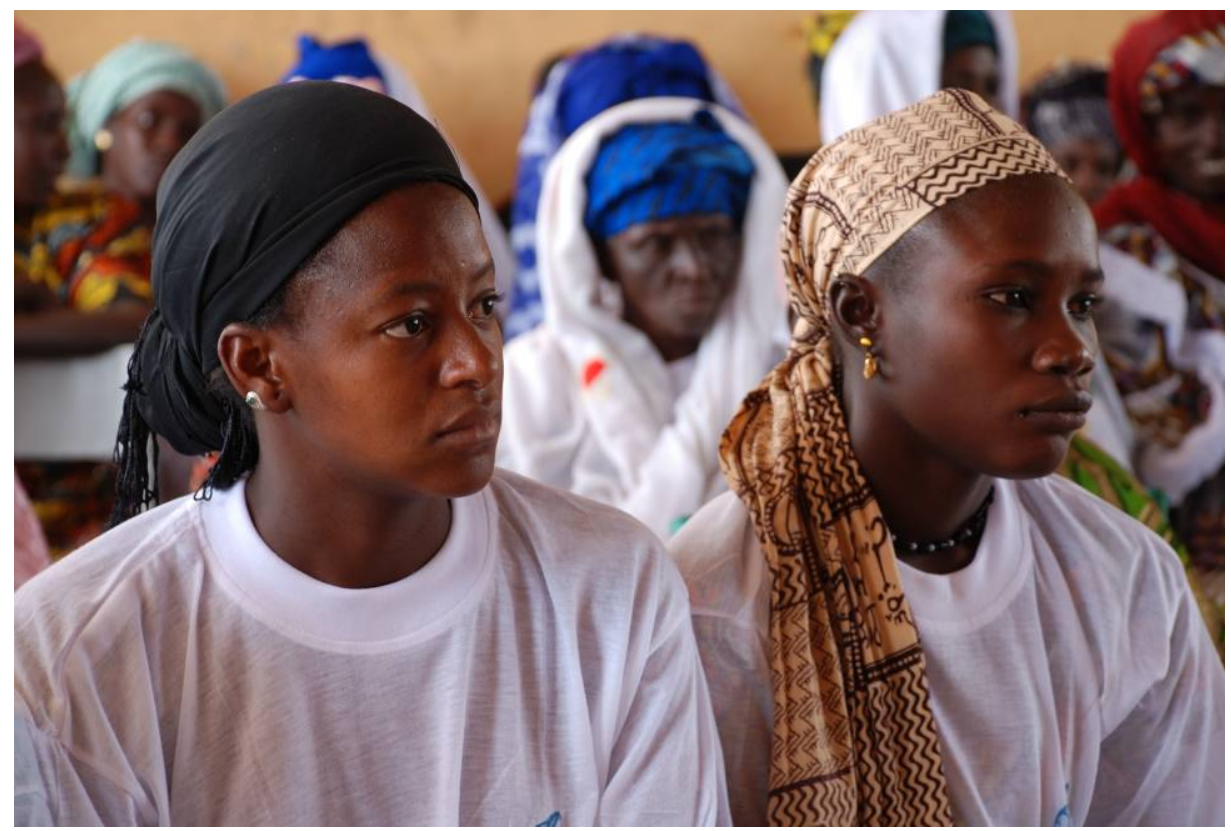

Il y a d'abord une relecture des causes réelles des drames survenus par le passé.

"Je pense que l'excision n'est pas bonne pour la santé. Personnellement, j'ai vécu des cas d'accidents (hémorragie) et l'enfant est décédé. Avant, les gens attribuaient cela aux sorciers mais de nos jours, la population a compris que c'est la pratique qui est à la base de ces accidents mortels." (Femme non participante, Kossédougou)

Par ailleurs, les drames familiaux personnellement vécus tels que le décès d'une sœur ou d'une fille constituent autant d'éléments négatifs qui sont mis au passif de l'excision :

"J'ai été personnellement victime d'un cas d'une de mes filles. Après une semaine sans laver ma fille excisée, j'ai trowvé que les lèvres du vagin étaient collées. Par la suite, elle en est morte. Après ce douloureux évènement, j'ai eu trois filles que je n'ai jamais excisées. Moi également, j'ai vécu un cas avec une de mes scours. Elle a été excisée à trois reprises, voilà un inconvénient. Un cas également vécu a été que la victime ne recevait pas de soins et les lèvres se sont collées. Ensuite elle avait toutes les peines du monde pour uriner et y mettait plus d'une heure. Alors, l'excision a été reprise mais en vain; le problème persiste et elle est en train de grandir avec ce problème urinaire." (Focus groupe femmes, Sananfara).

Ensuite, il émerge chez pratiquement tous les informateurs un phénomène de relativisation, de doute, voire de remise en cause des raisons socioculturelles et religieuses justifiant la coutume de l'excision :

"C'était autrefois qu'on disait que si une femme n'est pas excisée que si son clitoris touche le sexe de l'homme, il devient impuissant où au moment de l'accouchement si ça touche la tête de l'enfant ce dernier meurt où que la femme non-excisée ne peut pas avoir d'enfant. Mais tout cela, 
c'était autrefois, pas maintenant car on a vu des filles non-excisées qui ont eu des enfants sans problèmes et qui ont accouché. Et leurs enfants sont vivants. Tout ceci a montré aux gens que la non-excision n'est pas grave." (Jeune fille participante, Yanfolila).

Très peu d'informateurs ont fait allusion à certaines de ces raisons socioculturelles et religieuses pour expliquer leur attachement à leur coutume.

En définitive, l'amélioration du niveau des connaissances entrainant une relecture des causes réelles des complications passées et des drames familiaux survenus suite à l'excision a débouché sur une remise en cause des raisons justificatives de l'excision. Ce sont autant de facteurs qui ont contribué à augmenter le poids des inconvénients de la pratique de l'excision et, a contrario, à reconnaittre de plus en plus d'avantages à la non-excision.

Les avantages de la non-excision sont liés non seulement à la diminution des charges économiques entraînées par l'excision (paiement de l'exciseuse, soins, festivités), à la préservation de la santé de la jeune fille et de la femme (par rapport aux éventuelles complications et aux conséquences à long terme), mais aussi à l'harmonie affective et sexuelle du couple. Ce sont autant de facteurs liés au respect des droits de la petite fille, de la jeune fille et de la femme même si les informateurs n'évoquent pas de manière explicite dans leurs discours ce respect des droits comme avantage de la non-excision.

Ces témoignages illustrent bien ce ressenti :

"Ily a des changements. Tout d'abord, l'abandon de l'excision par tout le village. C'est la paix dans le village et les dépenses de santé sont réduites maintenant.» (Aide catéchiste, Sialo)

"Les avantages de la non-excision sont: tu es en paix, tu ne souffres pas de douleurs, tu n'auras pas de complications pour le mariage, tu vivras en paix contre les maladies que le matériel utilisé peut transmettre." (Jeune fille participante, Somo).

$\mathrm{Au}$ regard de tous ces avantages reconnus à la non-excision, on peut affirmer qu'une nouvelle vision émerge ainsi qu'une mutation des attitudes et des opinions des communautés par rapport aux jeunes filles et aux femmes non-excisées. De manière quasi unanime, les personnes interrogées ont noté un changement de comportements, de perceptions et d'opinions de la communauté - y compris chez les jeunes filles et des femmes excisées- vis-à-vis des jeunes filles et des femmes non-excisées. Cela se traduit par un jugement neutre sur leur statut, voire même par une certaine bienveillance. Les jeunes filles et les femmes excisées ont une nouvelle vision sur leur propre personne et leur personnalité.

Plus précisément, ces changements se vérifient à plusieurs niveaux :

- La communauté ne fait plus de discrimination à l'égard des jeunes filles et des femmes non-excisées qui, d'après les informateurs, participent à tous les évènements sociaux dans le village et peuvent accomplir tout ce que les autres femmes du village font sans ostracisme ni considération particulière :

"Les jeunes filles non-excisées ne sont pas exclues de la communauté. Elles peuvent être des responsables et elles peuvent prier comme toute autre personne." (Jeune fille participante, Somo).

- Le mépris et la stigmatisation à l'endroit des filles et des femmes nonexcisées tendent à disparaître: on les insulte de moins en moins en les traitant de «bilakoro» : 
Certains affirment que les hommes éprouvent plus de plaisir à avoir des relations sexuelles avec des femmes non-excisées. Ces personnes vont même jusqu'à affirmer que, dans un proche avenir, les hommes vont faire de la non-excision une exigence.
"Avant, on les appelait 'bilakoro2' mais plus maintenant et cette honte n'est plus d'actualité." (Exciseuse pratiquante, Pelengana).

'Tout le monde se méfie et même les enfants n'en parlent plus. Même si un parent surprend son enfant avec des critiques à l'encontre des filles non-excisées, il le sermonne et le met en garde contre toute palabre. Aussi, ces choses-là ne sont plus d'actualité de nos jours." (Focus groupe femmes, Sananfara).

- Les jeunes filles et les femmes excisées nourrissent de moins en moins de complexes de supériorité vis-à-vis des jeunes filles et des femmes nonexcisées et inversement, ces dernières ne se font plus de complexes d'infériorité; elles n'ont plus honte de leur statut et, selon certains informateurs, elles se sentent même fières de ce qu'elles sont:

"Elles sont bien perçues par les femmes excisées qui les considèrent comme plus complètes dans leur féminité." (Jeune fille non participante, Nousomna).

- En fin de compte, on pense que les hommes accepteront de les prendre pour épouses, cela signifie qu'elles auront les mêmes chances (voire même plus de chances) de trouver un mari que les jeunes filles et femmes excisées :

"Les femmes qui ne sont pas excisées sont la préférence des hommes d'aujourd'bui. Aussi, la non-excisée a plus de chance de s'acquérir un mari. D'ailleurs, depuis leur bas âge, elles sont fiancées par des hommes en attendant d'avoir l'âge du mariage." (Focus groupe femmes, Sananfara).

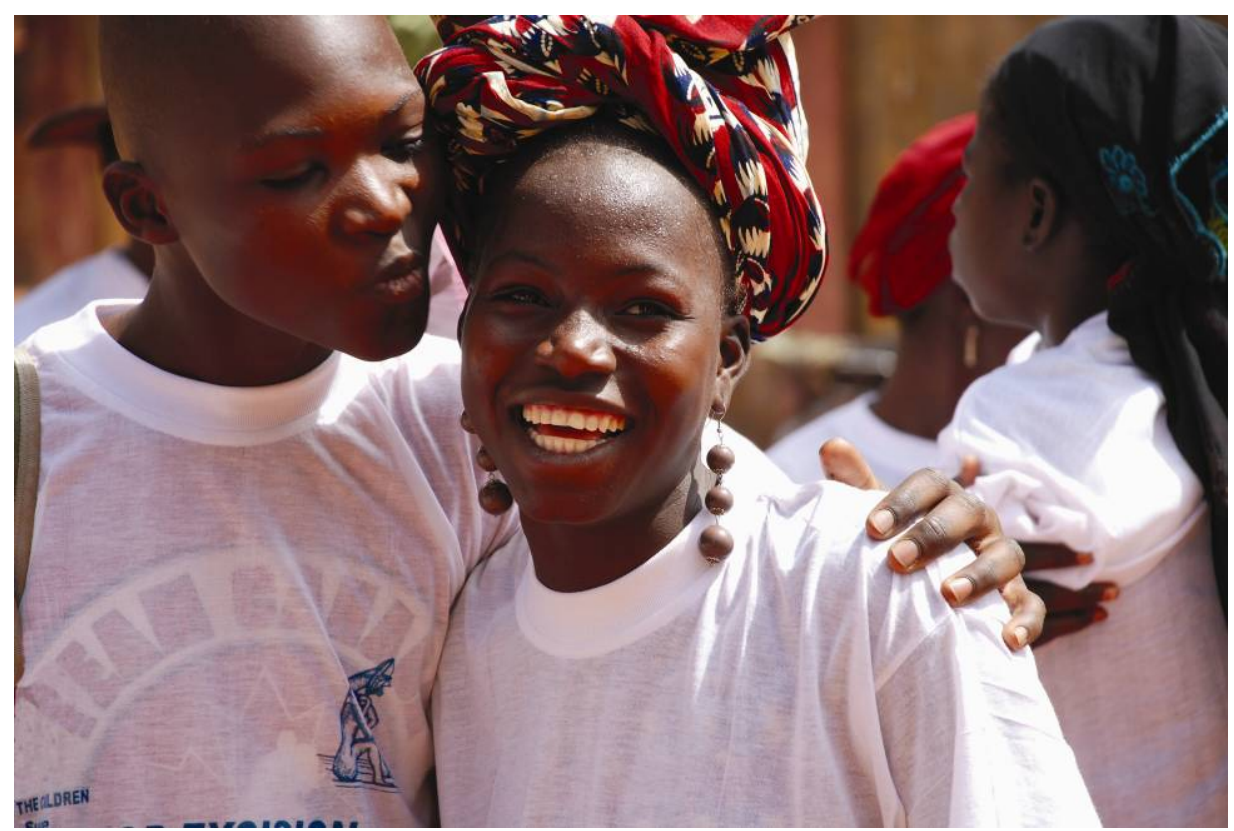

En résumé, il apparaît que les jeunes filles et les femmes non-excisées bénéficient d'avantages liés à la non-excision. Un aspect important qui pourrait avoir à l'avenir un caractère décisif du point de vue des mutations dans les attitudes vis-à-

2 Personne non initiée, considérée comme un enfant 
vis des jeunes filles et des femmes non-excisées est celui de la sexualité et du changement du statut social accordé aux femmes non-excisées.

En effet, certains affirment que les hommes éprouvent plus de plaisir à avoir des relations sexuelles avec des femmes non-excisées. Ces personnes vont même jusqu'à affirmer que, dans un proche avenir, les hommes vont faire de la nonexcision une exigence et que les jeunes filles excisées auront alors du mal à trouver un époux :

"Bien sûr que les hommes accepteraient d'épouser une fille non-excisée. C'est d'ailleurs, l'une de leur préférence qui ne va pas tarder à être une exigence. Les raisons, c'est les hommes qui le savent. Et ce sont surtout des raisons sexuelles et sentimentales car les hommes l'affirment haut et fort qu'ils éprouvent plus de sentiments avec une fille non-excisée qu'avec celle excisée." (Focus groupe femmes, Sananfara).

La conséquence logique au plan comportemental et cognitif est que la situation des filles et femmes non-excisées commence à susciter l'envie des jeunes filles et des femmes excisées. C'est ce qu'exprime en des termes très forts une participante du focus groupe avec les femmes adultes de Sananfara :

"Nous-mêmes, vieilles femmes excisées, voudrions revenir en arrière pour être non-excisées. (...) Moi, ma coépouse se moque régulièrement de mon statut de femme excisée. Une fois, lors d'une dispute, mon mari même m'a dit de laisser ma coépouse en paix car celle-là le satisfait mieux au lit que moi qui suis excisée. (...) Les non-excisées bénéficient d'une considération et prestige mieux que les excisées. Elles ne sont plus victimes de discrimination et peuvent s'exprimer haut et fort partout où elles veulent. D'ailleurs, elles se vantent de leur statut de femme non-excisée." (Focus femmes, Karo).

Il n'est pas exagéré d'affirmer qu'il y a là un véritable changement de paradigme. En effet, par le passé, les hommes n'avaient pas beaucoup d'expérience sexuelle avec une femme non-excisée. Aujourd'hui, comme il y a de plus en plus de femmes mariées non-excisées qui remplissent leurs devoirs conjugaux et qui satisfont sexuellement leur époux, ces derniers commencent à en faire état.

Dans une société où le statut de la femme est principalement valorisé selon qu'elle est mariée, le fait qu'on découvre que la femme non-excisée ne répugne pas à remplir ses devoirs conjugaux, mais encore qu'elle est en mesure de combler sexuellement son mari est un changement non négligeable. Par conséquent, alors que l'excision était considérée comme une condition sine qua non du mariage pour diverses raisons, elle apparaît peu à peu comme un obstacle à l'harmonie sexuelle des couples et donc comme une menace pour la stabilité des ménages et des familles.

Tous ces facteurs font que les populations remettent de plus en plus en cause le bien fondé de la pratique de l'excision et que, dans les communautés encadrées par le Centre Djoliba, les populations se mobilisent de plus en plus pour son abandon. 
La prise de décision au sein de la famille revient à l'homme qui en est le chef. Ainsi, même pour l'excision des filles, ce sont les hommes qui décident bien que certains d'entre eux aient affirmé que (...) la circoncision est l'affaire des hommes et l'excision l'affaire des femmes.

Dans certains villages, ce sont les vieilles femmes qui s'imposent à leurs fils et font exciser leurs petites filles. Les exciseuses, les mères des filles et les filles elles-mêmes n'ont pas de pouvoir de décision.

\section{Description du processus d'abandon ou de régression}

Selon que le processus de régression de la pratique est parvenu à son stade ultime ou non, on peut parler d'abandon total de l'excision, de pratique en nette régression ou alors de résistance. A l'analyse des données, il apparaît deux cas de figure à savoir l'abandon total ou alors la nette régression de la pratique. Ainsi, la "situation minimum" admise par toutes les catégories d'informateurs, quel que soit le village considéré, est que la pratique de l'excision est en régression.

\section{a. Les instances de décision d'abandon}

Dans le cadre de la mobilisation pour l'abandon de l'excision, il est intéressant de comprendre la question des instances de décision ou des personnes détentrices du pouvoir de décision.

Les instances de décision identifiées au niveau communautaire sont le chef de village et ses conseillers, les chefs traditionnels, les leaders religieux, les leaders des femmes et des jeunes. Dans les villes, par exemple les chefs-lieux de préfectures, l'administration intervient en plus des autorités villageoises. Comme partout ailleurs, il y a des instances, des lieux ou des occasions de concertation, de discussion et d'échanges entre les différents membres sur toute question de développement et de fonctionnement des communautés, néanmoins la décision finale revient souvent aux leaders hommes.

Par rapport à la pratique de l'excision, il apparaît que c'est le chef de village et les autres leaders qui décident de la période d'excision et informent l'exciseuse. Cette dernière ne peut donc venir exciser dans le village sans l'ordre du chef. Cela montre que l'acceptation de l'intervention du Centre Djoliba aussi bien que la décision d'abandon ne peuvent se faire sans l'aval du chef de village et des leaders hommes en général et religieux en particulier. Ces derniers en effet détiennent un pouvoir particulier dans la mesure où l'excision est souvent envisagée comme une exigence religieuse.

"L'implication des chefs de famille a été déterminante dans l'abandon de l'excision car ce sont eux qui prennent les grandes décisions de la famille. Il y a des familles qui ont abandonné la pratique, il y a de cela 20 ans et elles "possèdent 》 des jeunes filles de cet âge non-excisées." (Focus groupe femmes Sananfara).

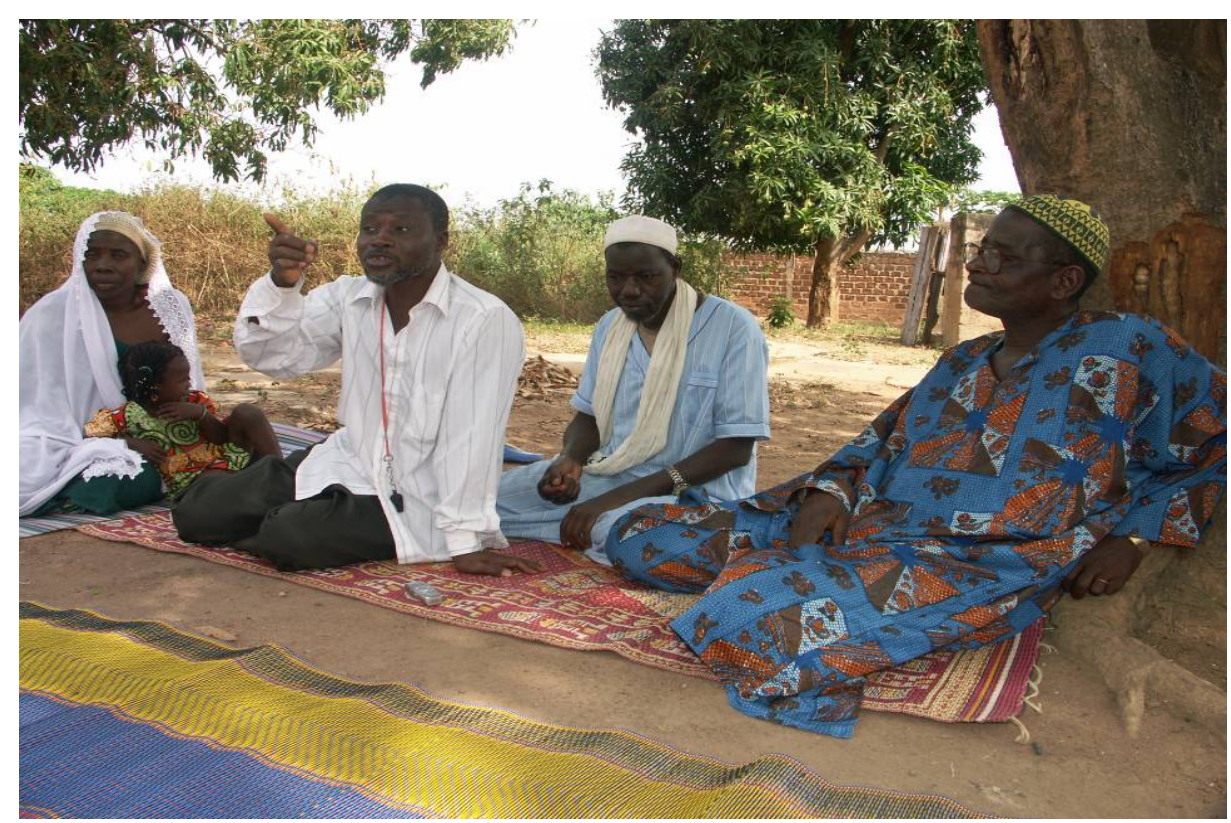


La prise de décision au sein de la famille revient à l'homme qui en est le chef. Ainsi, même pour l'excision des filles, ce sont les hommes qui décident bien que certains d'entre eux aient affirmé au cours des interviews que "la circoncision est l'affaire des hommes et l'excision l'affaire des femmes" et que les dépenses des festivités reposent essentiellement sur les femmes.

De plus en plus, avec l'entrée de l'excision dans la clandestinité dans certains villages, ce sont les vieilles femmes qui s'imposent à leurs fils et font exciser leurs petites filles (cas de Yanfolila où les vieilles femmes et les exciseuses font partie des résistantes). Les exciseuses, les mères des filles et les filles elles-mêmes n'ont pas de pouvoir de décision dans la pratique de l'excision.

On se rend compte alors des enjeux selon que les leaders sont favorables ou au contraire hostiles à l'abandon de l'excision et du bien-fondé de la stratégie consistant à recruter et à former les leaders hommes, et à sensibiliser les chefs de familles dans le cadre des interventions visant l'abandon de la pratique de l'excision.

\section{b. Les éléments précurseurs}

Le processus conduisant à l'abandon de l'excision s'est appuyé sur un élément essentiel qui est, rappelons-le, la remise en cause de la raison d'être même de cette pratique, suite à l'accès à une information plus systématisée et complète sur ses conséquences négatives et à la confirmation de ces mêmes effets néfastes par des drames personnels ou familiaux vécus.

En effet, au moment de l'implantation du programme d'intervention, les communautés sont réticentes, voire très hostiles pour certaines. Mais au fur et à mesure que l'information circule avec la sensibilisation tous azimuts, le niveau de connaissance augmente, la réflexion et la prise de conscience individuelle et collective des inconvénients de l'excision commencent à peser lourd sur la balance en faveur de l'abandon.

A cela s'ajoute le fait que les communautés sont de plus en plus informées que l'excision est un phénomène qui fait l'objet d'une attention particulière aux niveaux national et international et qu'au-delà des frontières du pays, il existe un fort mouvement abolitionniste.

"Vu que l'excision constitue un thème d'actualité mondiale, vu qu'elle n'a pas de rapport avec l'Islam et vu ses conséquences sur la santé de la femme, le processus fut accepté en concertation avec tout le village." (Chef de village Kossédougou).

\section{c. Le consensus vers le changement}

A la faveur des facteurs déclenchant évoqués plus haut, le consensus pour l'abandon de l'excision s'élargit peu à peu à l'ensemble de la communauté. En général, il émerge d'abord des groupes ou des personnes qui ont bénéficié d'une formation systématisée ou qui sont arrivés au bout du processus de remise en cause de la pratique. Ces groupes ou personnes (des femmes responsables ou membres d'associations, des chefs de famille, de jeunes couples, des jeunes, des leaders catholiques, etc.) forment le noyau représentant le leadership en matière d'abandon. Au tout début, ces personnes ont cru et adhéré à l'objectif d'abandon et font en sorte que leurs convictions gagnent peu à peu d'autres groupes de la communauté. Dans beaucoup de sites, les leaders religieux et les exciseuses ont joué un rôle important dans le processus vers le changement.
Le processus conduisant à l'abandon de l'excision s'est appuyé sur (...) la remise en cause de la raison d'être même de cette pratique, suite à l'accès à une information plus systématisée et complète sur ses conséquences négatives et à la confirmation de ces mêmes effets néfastes par des drames personnels ou familiaux vécus. 
Les leaders religieux ont été les premières cibles des interventions du Centre Djoliba (...) Ce contact a permis d'obtenir leur ‘bénédiction' et donc leur implication dans le processus.

\section{Les exciseuses}

appartiennent à la caste des forgerons et on leur prête des pouvoirs mystiques et surnaturels qui sans doute participent du respect mêlé de crainte dont elles jouissent dans leur communauté.
- Adhésion et engagement des leaders religieux

Dans la plupart des sites visités, les leaders religieux ont été les premières cibles des interventions du Centre Djoliba dans la mesure où ils ont été consultés dans la sélection de leur village comme site d'intervention du Centre Djoliba. Ce contact a non seulement permis de les informer sur les objectifs du Centre Djoliba mais aussi d'obtenir leur «bénédiction » et donc leur implication dans le processus. Ensuite les leaders religieux ont bénéficié de séances de sensibilisation et de formation sur le thème.

$\mathrm{Si}$, au départ, la plupart des leaders religieux avouent leur méconnaissance des effets néfastes de l'excision et leur réticence face à la lutte contre son abandon, ils affirment être devenus des acteurs de la sensibilisation des membres de leurs communautés.

"Au départ, certains religieux étaient pour la pratique mais, avec la déclaration d'abandon suite aux différentes sensibilisations et informations, ils ont adhéré à l'abandon.» (Leader religieux, Nousomna).

La position de ces leaders religieux montre qu'il est possible de gagner l'approbation, l'adhésion et l'implication de ce groupe en les sensibilisant et en renforçant leurs compétences sur la question au même titre que le reste de la communauté.

- L'engagement des exciseuses dans la mobilisation pour l'abandon de l'excision

Les exciseuses constituent un élément très important dans le processus d'abandon en ce sens que, selon leurs convictions et leurs motivations, elles peuvent être des éléments catalyseurs ou au contraire constituer un blocage. Le Centre Djoliba a mené avec les exciseuses des activités de renforcement des capacités, de séances d'IEC, mais les a aussi soutenues et organisées en associations. La plupart des informateurs ont relevé le fait que les exciseuses ont affirmé avoir arrêté leurs activités pour plusieurs raisons telles que la formation et les séances d'IEC dont elles ont bénéficié, la peur d'être réprimées et le fait qu'elles ne sont plus sollicitées consécutivement à la déclaration d'abandon.

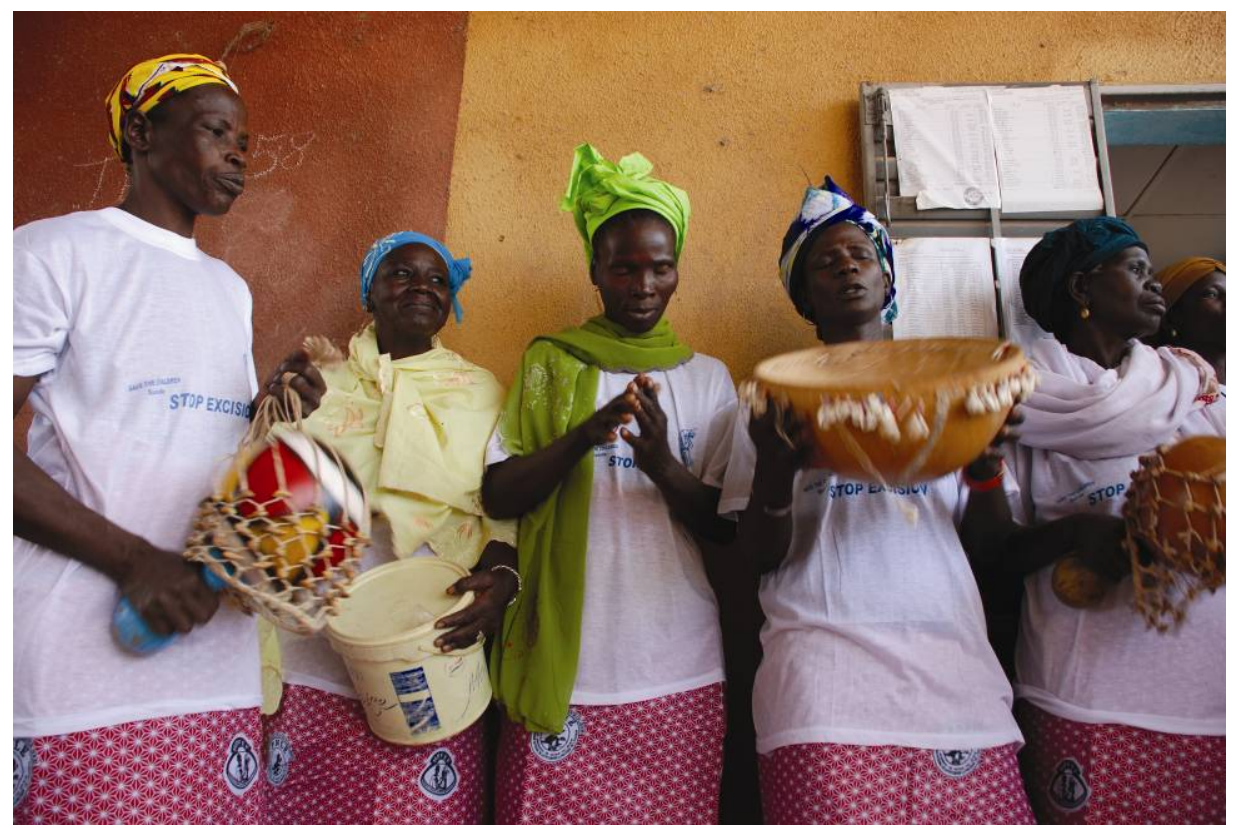


Les exciseuses appartiennent à la caste des forgerons et on leur prête des pouvoirs mystiques et surnaturels qui sans doute participent du respect mêlé de crainte dont elles jouissent dans leur communauté.

Par ailleurs, elles ont une situation économique assez enviable au vu des revenus financiers et en nature que leur procurent leurs activités.

Les exciseuses qui ont abandonné la pratique de l'excision se sont engagées dans la mobilisation contre l'excision. Il y a eu un effort de sensibiliser, de mobiliser et d'organiser les exciseuses. Elles ont été spécifiquement ciblées à travers les objectifs stratégiques des interventions du Centre Djoliba.

Cette option semble avoir porté ses fruits puisqu'il existe des exciseuses qui ont abandonné la pratique et qui se sont regroupées en associations. Des membres d'associations d'ex-exciseuses ont mis en exergue les efforts que le Centre Djoliba déploie et le rôle qu'il a joué dans la mise sur pied de leurs associations.

Cependant, le fonctionnement de ces associations n'est pas toujours jugé satisfaisant. Si l'association des ex-exciseuses de Pelengana semble fonctionner relativement bien, il n'en est pas ainsi de celle de Kossédougou. C'est ce que regrette un des membres en ces termes :

"L'association ne fonctionne plus après la formation du Centre Djoliba puisque pas de financement ni d'activités à mener (...). Nous avons été regroupées par le Centre Djoliba pour une formation au cours de laquelle il nous a donné 4000 francs $C F A$ par personne. Le Centre Djoliba nous a promis de nous appuyer dans les activités d'animation à travers des séances de causeries (sensibilisation/information) et des scènes de théâtre, mais après la formation plus rien."(Membre Association ex exciseuses, Kossedongou).

La création de ces associations vise notamment un objectif de reconversion professionnelle de ces ex-exciseuses par le biais des activités génératrices de revenus. Pour ce faire, leurs membres bénéficient d'un renforcement de capacités sur différents aspects en lien avec la mobilisation contre l'excision et sur d'autres thèmes en rapport avec le développement communautaire. Selon la présidente de l'association des ex-exciseuses de Pelengana, les membres sont impliqués dans la mobilisation avec un certain succès :

"Notre association a été créé il y a de cela 5 ans environ. Nous menons des sensibilisations pour la communauté afin de l'informer sur les inconvénients et les méfaits de l'excision (...). Dans la lutte contre l'excision, nous communiquons avec toute la communauté (femmes, hommes, jeune et personnes âgées) et aussi avec les exciseuses pratiquantes (...). Notre statut d'ex-excisenses converties en animatrices de sensibilisation contre la pratique marque beaucoup la population et constitue un facteur déterminant dans la prise de conscience de la communauté contre la pratique. » (Présidente de l'association des ex-exciseuses, Pelengana).

Les ex-exciseuses interrogées déclarent fermement que jamais plus elles ne reprendront leur ancienne activité. 


\section{Témoignage d'une ex-exciseuse de Kossédougou}

Suite aux informations reçues du Centre Djoliba et en comparant les inconvénients de l'excision par rapport aux avantages de la non-excision, j'ai décidé et avec moi d'autres pratiquantes, d'arrêter la pratique de l'excision. Au départ, nous avions des problèmes puisque la population pensait qu'on nous avait payées pour médire l'excision et encourager l'abandon de la pratique. Certains boudaient nos séances de causeries et d'animations. Le Centre Djoliba nous a toujours encouragées de continuer en nous offrant des motivations à travers nos animations. D'autres ex-exciseuses sont devenues des actrices (Djanfola) pour compenser leurs revenus.

J'ai rencontré des difficultés financières et économiques car j'ai perdu ma seule source de revenus, ce qui constitue un problème majeur dans l'entretien de ma famille et le paiement de mes impôts. Je suis toujours très sollicitée par les villages voisins mais je ne cèderai jamais (...). Un village voisin m'a sollicitée par l'intermédiaire de notre chef du village pour venir pratiquer l'excision des jeunes filles de leur village. Malgré leurs prières et leur insistance, je n'ai pas cédé à leur demande et leur ai dit que la pratique est abandonnée et interdite. (...) Rien ne pourrait plus jamais m'amener à reprendre le couteau de l'excision car convaincue des inconvénients de la pratique. Je participe aux activités de sensibilisation, d'éducation et d'informations de la population sur la lutte contre l'excision avec le refus catégorique de reprendre la pratique. D'autres exciseuses (07) ont abandonné et arrêté la pratique en rejoignant notre association. Aujourd'hui, l'association compte 22 personnes au total.

Il faut des formations pour la population enfin de la sensibiliser davantage sur les méfaits de la pratique de l'excision. Il faut également de la persévérance de la part des animateurs afin d'atteindre nos objectifs.

Il semble que les communautés soient sensibles à la situation économique des exciseuses, et c'est ainsi que des initiatives sont notées ça et là pour leur venir en aide.

"L'exciseuse du village était ma grand-mère. A travers nos causeries, elle a arrêté la pratique 3 ans avant de mourir. Quand on lui disait d'arrêter, elle nous demandait si nous allions la prendre en charge. Nous cotisions chaque mois 10.000 francs $C F A$ pour elle afin qu'elle puisse arrêter. A la fin, elle nous accompagnait au cours de nos animations et intervenait en donnant des exemples concrets et des cas désastreux rencontrés." (Femme leader, Sananfara).

Cela étant, la stratégie de reconversion des ex-exciseuses par les activités génératrices de revenus et leur regroupement en association peuvent se révéler être une arme à double tranchant. On risque en effet d'installer les ex-exciseuses dans une position de chantage sur les communautés qui veulent abandonner, mais aussi sur les $\mathrm{ONG}$ et structures qui les encadrent en menaçant de reprendre le couteau si elles ne reçoivent pas des financements; ce qui à long terme n'est pas réalisable. Avec le dépôt des couteaux, elles sont confrontées à un manque à gagner, et on peut se demander jusqu'à quel point elles arriveront à résister aux sollicitations qui leur sont adressées par les communautés qui n’ont pas abandonné la pratique de l'excision.

Par conséquent, il est important pour le Centre Djoliba d'insister sur son approche qui considère les ex-exciseuses comme toutes les autres femmes de la communauté. Il devrait également les amener au même titre que ces femmes à être convaincues de la nécessité de l'abandon de la pratique de l'excision et de l'amélioration de leur statut en tant que femme.

En synthèse, il faut retenir que le consensus est le résultat d'un processus plus ou moins long de discussions et de concertations entre le Centre Djoliba, les 
autorités villageoises et les chefs de familles ou parfois entre le Centre Djoliba, les autorités villageoises et le groupe initiateur de l'abandon. Il peut être concrétisé ou non par une déclaration d'abandon. Il peut aboutir à l'abandon (consensus total) ou à la régression de la pratique dans le village sans qu'il y ait eu passage par la déclaration d'abandon. Signalons qu'au sein d'un même village, les informations à propos de l'effectivité d'une déclaration d'abandon sont contradictoires selon les catégories d'informateurs. Il est cependant possible d'affirmer que les sites suivants ont effectivement fait une déclaration de l'abandon de l'excision: Banankourou, Kossédougou, Nousomna, Karo, Sialo, Somo.

Selon le cas i) la déclaration est "supra communautaire" en ce sens qu'elle a lieu dans le chef-lieu de commune à laquelle est rattaché le village ; ii) elle est faite par le chef de village et ses conseillers au cours d'une assemblée du village, et chacun est invité à y adhérer; iii) dans certains cas, elle est faite par des groupes "coalisés" (les femmes, les jeunes, des chefs de famille, etc.) iv) ou encore par tout le village. En tout état de cause, la déclaration de l'abandon est un processus libre.

Une fois que le consensus a abouti à la déclaration ou à une tendance à l'abandon, il s'en suit un regain de mobilisation totale ou partielle de la communauté pour l'abandon de la pratique, surtout chez les femmes. Dans certains cas également, il y a un "effet de contagion" qui se traduit par la sollicitation des relais des villages d'abandon pour des séances d'animation dans d'autres villages environnants, ou par le fait que des villages voisins se joignent à ce mouvement et prennent eux aussi la décision d'abandonner. Cependant, les informateurs ne sont pas catégoriques sur l'effectivité de l'abandon dans ces villages- là.

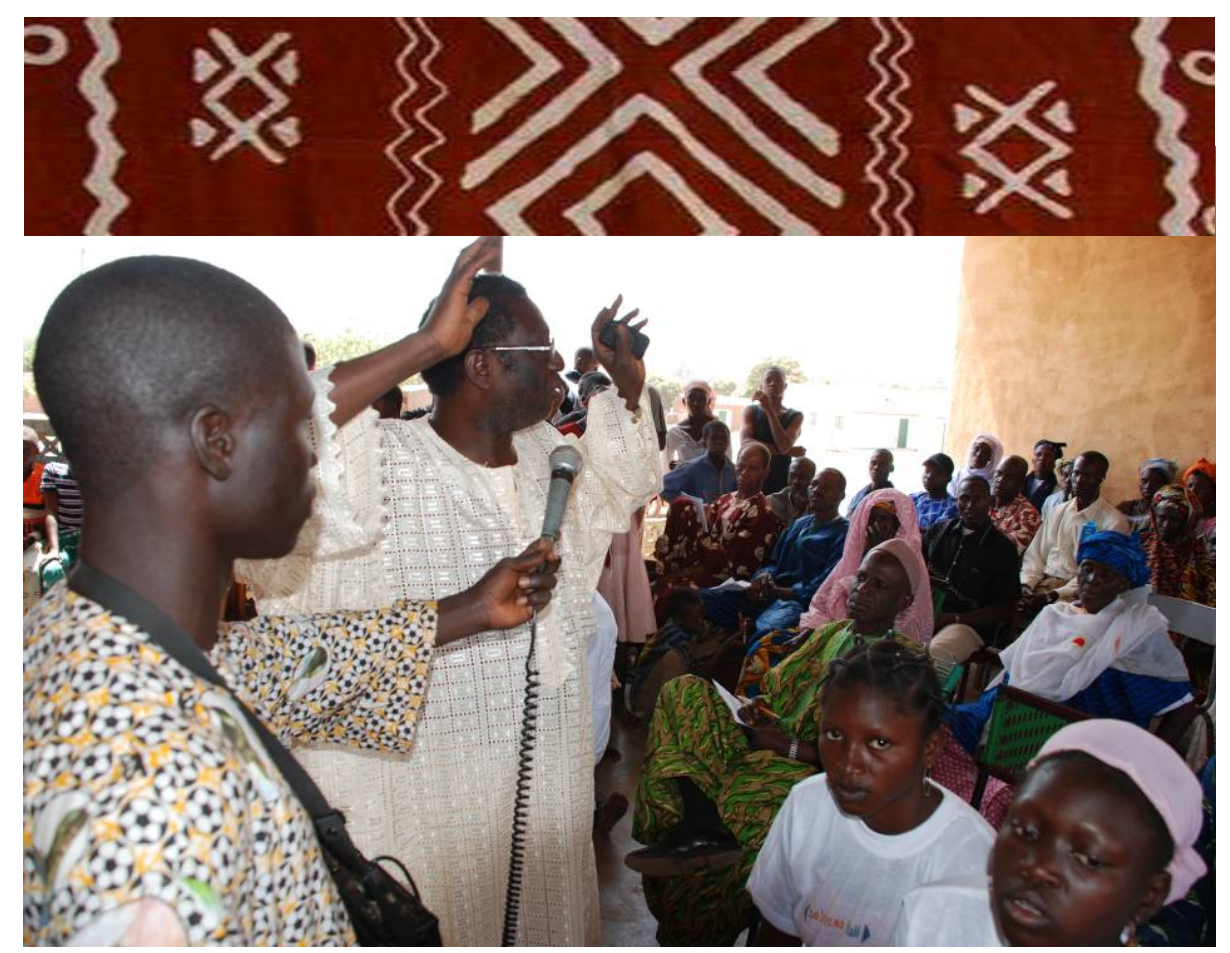

Il convient cependant de relever, dans certains villages, une "attitude d'attente" qui accompagne l'abandon de l'excision qu'il soit total ou non. Dans ces villages, les communautés qui abandonnent semblent vouloir attendre de constater concrètement chez les jeunes filles et les femmes non-excisées tous les bienfaits qu'on attribue à la non-excision. C'est ce qui ressort des déclarations suivantes : 
Dans certaines localités où l'abandon de la pratique de l'excision est important, les villageois ont mis sur pied des comités de surveillance ou de vigilance ayant pour mission de s'assurer du respect de la déclaration d'abandon de la pratique.

"Si une décision est prise dans notre village, tout le monde respecte cette décision. Personne dans le village n'ose défier la décision commune." (Femme non participante, Goualala).
"L'abandon est une réalité dans notre village depuis la déclaration d'abandon. Les filles nonexcisées sont petites, nous attendons de voir les résultats de la non-excision quand les filles seront plus grandes et mariées, si elles auront des enfants." (Femme participante, Karo).

\section{d. Le suivi de la décision d'abandon}

Qu'il y ait déclaration d'abandon ou pas, le consensus autour de l'abandon de l'excision est généralement suivi d'efforts de respecter la décision d'abandonner la pratique et de surveiller l'évolution de cet abandon dans la communauté. Le suivi se fait de manière formelle ou informelle selon le cas.

\section{- Suivi communautaire organisé}

Dans certaines localités où l'abandon de la pratique de l'excision est important, les villageois ont mis sur pied des structures ad-hoc que sont les comités de surveillance ou de vigilance ayant pour mission de s'assurer du respect de la déclaration d'abandon de la pratique. Il existe des comités de surveillance dans 5 villages : Banankourou, Karo, Kossédougou, Sialo et Nousomna. A noter que l'existence d'un comité de surveillance ne coïncide pas toujours avec une situation d'abandon total dans le village. Parfois, le comité de surveillance est mis sur pieds à l'initiative du Centre Djoliba. Selon les villages, il semble que le suivi se fasse de manière plus ou moins rigoureuse :

"Le comité est crée en vue de surveiller les excisenses dans leurs activités. Nous menons également des sensibilisations sur l'excision et la promotion de la santé de l'enfant." (Membre du comité de surveillance, Banankourou).

"Le comité surveille tous les bébés (filles) à leur naissance au centre de santé communautaire (siège du comité) pour confirmer la non-excision. Ce contrôle se fait concrètement en observant le sexe de l'enfant. Pour les jeunes filles, ce sont les membres féminins du comité qui font le contrôle." (Leader du comité de surveillance, Kossédougou).

La surveillance par les leaders se fait avec l'implication et l'engagement du maire, du chef de village et/ou ses conseillers, des relais communautaires, des agents de santé, des matrones/accoucheuses traditionnelles, des groupements de femmes.

\section{- Suivi communautaire non organisé}

Dans d'autres villages, la surveillance et le suivi ne se font pas par le biais de personnes ou de structures identifiées, mais par la communauté dans son ensemble. Les mécanismes d'un tel suivi sont fondés sur le mode de vie villageois où rien ne peut se passer à l'insu des autres et sur les principes de respect de la parole donnée et de l'autorité du chef.

"Si une décision est prise dans notre village, tout le monde respecte cette décision. Personne dans le village n'ose défier la décision commune." (Femme non participante, Goualala).

"La violation de la déclaration d'abandon est considérée dans le village comme une atteinte à l'autorité du chef de village." (Chef de village, Karo).

\section{e. Tendance future}

Mais au-delà de l'impact que pourrait avoir le suivi organisé ou non de l'abandon de la pratique de l'excision, on peut retenir comme gage d'un abandon effectif de la pratique de l'excision à plus ou moins longue échéance, les intentions fermement exprimées par les jeunes filles interrogées de ne pas exciser leurs (futurs) enfants. Qu'elles aient pris part aux activités de sensibilisation ou non, les jeunes filles se sont déclarées opposées à cette coutume et n'entendent pas (à 
quelques rares exceptions près) faire exciser leurs filles, quelle que soit la pression sociale subie venant du mari ou de la belle-famille.

"Dans l'avenir, mes enfants ne seront pas excisées pour qu'elles ne soient pas malades et j'userai de tout auprès de mon époux et de ma belle-mère pour que cela soit ainsi." (Jeune fille participante, Yanfolila).

Il est vrai que la question des intentions futures des jeunes gens et des hommes par rapport à l'excision de leurs filles ne leur a pas été posée spécifiquement, mais selon la majorité des informateurs, l'excision est une pratique qui va disparaitre dans un avenir plus ou moins proche car le nombre de ceux qui y sont opposés s'accroit de plus en plus et ils finiront par prendre le dessus. Pour eux, c'est juste une question de temps et ils s'appuient sur les changements qu'ils ont constatés entre le début de l'intervention du Centre Djoliba à maintenant.

"Il peut arriver un jour où dans la communauté on ne parlera plus de la pratique de l'excision. C'est bien possible. Il existe des filles non-excisées dans la communauté et leur nombre commence à augmenter avec l'intervention du Centre Djoliba. Elles sont nombreuses car ici, c'est l'abandon total et collectif. Et nous pensons qu'elles resteront comme cela." (Focus groupe hommes, Banankourou).

Même dans les sites de résistance, certains informateurs ont souligné la tendance inéluctable vers l'abandon de l'excision, à plus ou moins longue échéance. Les changements de comportement qui se sont opérés de par le passé par rapport à la planification familiale justifient cette tendance:

"Pour moi, l'excision va disparaître un jour car il y a des situations plus difficiles notamment le planning familial, quand on disait espacer les naissances en prenant la pilule ou en faisant les injections, ceci était incroyable. Mais maintenant les femmes font le planning familial sans problème de même que l'utilisation du préservatif pour prévenir le SIDA mais là aussi le préservatif est utilisé sans problème donc tôt ou tard, l'excision aussi sera abandonnée car si la sensibilisation continue au fil du temps les gens vont abandonner car les gens auront compris l'importance de cela." (Jeune fille non participante, Yanfolila).

\section{f. Catégorisation des sites selon le degré d'abandon}

Il est possible de classer les douze sites d'intervention selon ces trois modalités : sites d'abandon total de l'excision, sites de (nette) régression et enfin sites de résistance à l'abandon.

Les sites d'abandon sont Kossédougou, Somo, Sialo et Banankourou. Pour ces villages d'abandon effectif de l'excision, il y a des facteurs liés aux activités du Centre Djoliba. Pour le village de Somo par exemple, le fait que le chef de village et le maire de la commune aient été formés et jouent le rôle de relais communautaires, a été un élément décisif dans le processus d'abandon.

Il s'y ajoute des "facteurs favorisants" tels que :

- l'implication de certains leaders religieux dans la sensibilisation contre l'excision avant le début de l'intervention sur l'approche droits de l'enfant (certainement parce qu'ils ont été formés auparavant) ;

- l'existence dans le substrat culturel de la communauté de coutumes en faveur de la non-excision. C'est le cas du village de Kossédougou :

- pour certains villages, l'excision n'était pas initialement une tradition du village. Elle a été introduite avec l'islamisation; il s'agit de villages animistes et/ou chrétiens. 
Les sites de régression sont Katabankoto, Nousomna, Kati et Karo. Ce sont des sites d'intervention pour lesquels il est difficile d'affirmer que toute la communauté a abandonné l'excision; les informateurs eux-mêmes reconnaissent que si la majorité a abandonné, il demeure tout de même des réticents :

"Ceux qui n'ont pas abandonné vont le faire un jour parce qu'ils ne le font plus au vu et au su de tout le monde. Ils le font clandestinement. Donc un jour, ils vont abandonner." (Focus groupe hommes et femmes, Gualala).

Dans ces sites, les résistants adoptent des stratégies de contournement :

- ils se cachent pour exciser leurs enfants, il y a donc une disparition de l'excision en groupe et des festivités qui l'accompagnent ;

- certains villageois décident de quitter temporairement leur village à cause de l'abandon déclaré ;

- d'autres excisent à un âge de plus en plus précoce (bébé).

Les sites de résistance sont Gualala, Pelengana et Yanfolila. Ce sont des localités où ceux et celles qui pratiquent encore l'excision sont bien plus nombreux que ceux et celles qui l'ont abandonné et ces derniers se comptent en petit nombre. La plupart des habitants pratiquent l'excision ouvertement.

Par ailleurs, pour ce qui concerne Yanfolila, il semble qu'il y a encore des problèmes pour communiquer sur le sujet. En effet, les femmes avaient protesté contre une radio locale suite à une émission réalisée par une autre ONG.

De manière générale, la résistance est le fait des religieux musulmans, des personnes âgées (surtout les femmes âgées) et des exciseuses. En plus des arguments religieux qu'ils avancent, on sait que les résistants accusent parfois les autres de renier leur coutume rien que pour un profit matériel.

"L'excision est un fait divin. C'est Dieu qui a recommandé à Ibrabima de le faire lorsqu'il avait plus de 70 ans avec sa femme Adiara. Dieu recommande à ses fidèles de suivre la voie d'Ibrabima. La nouvelle conception qui tend vers l'abandon [...] n'est pas conforme à l'Islam." (Focus groupe, Nousomna).

"Je suis pour la pratique de l'excision car c'est une tradition qui dure de longue date et j'aimerais qu'elle puisse continuer à se pratiquer. (...) Je ne connais pas les avantages ni les inconvénients de la non-excision mais tout ce que je sais, c'est que je pratique l'excision pour respecter ma tradition." (Femme non participante, Pelengana).

\section{Autres impacts de l'intervention du Centre Djoliba}

Au-delà de la régression ou de l'abandon de la pratique de l'excision qui peut être considéré comme le premier impact, l'analyse des informations recueillies auprès des différentes catégories d'informateurs a montré que l'intervention du Centre Djoliba dans les villes et villages a entrainé des effets significatifs qui peuvent s'analyser en termes de changements positifs menant vers l'amélioration des conditions de vie des populations en général. 


\section{a. Emergence d'un leadership des femmes}

Nombre d'informateurs ont relevé l'encadrement dont les associations féminines ont bénéficié de la part du Centre Djoliba. En effet soit le Centre Djoliba a été directement à l'origine de la création d'associations, soit il a appuyé la redynamisation de celles qui existaient déjà. C'est ainsi que les femmes membres de ces groupements ont bénéficié d'un renforcement des capacités dans plusieurs domaines tels que la santé de la reproduction (excision, IST/SIDA, PF), la santé maternelle et infantile, les droits de l'enfant, l'hygiène et l'assainissement etc., avec notamment comme objectif de faire d'elles des agents de sensibilisation au sein de la communauté.

"L'intervention du Centre Djoliba a beaucoup aidé dans l'organisation et la structuration de notre association, il y a eu plus de femmes qui ont adhéré à l'association et l'intégration de nouvelles activités comme la tontine et l'entraide au sein de l'association, notre capacité de mener des séances de sensibilisation a été améliorée et nos connaissances se sont développées avec beaucoup d'informations rȩ̣ues." (Femme participante, membre de l'ONG Tagnè).

"Le Centre Djoliba nous a aidées en nous organisant en association. Nous sommes vraiment beaucoup sollicitées à travers nos différents domaines d'intervention à savoir la lutte contre les IST/VIH/SIDA et la lutte contre l'excision. Aussi, nous venons de recevoir un prix du Ministère de la Promotion de la Femme, de l'Enfant et la Famille. Nous dédions ce prix au Centre Djoliba car c'est grâce à sa collaboration que nous avons pu recevoir ce prix." (Femme participante, Kati)

.D'après ces femmes, les interventions du Centre Djoliba ont eu comme effets une prise de conscience accrue de leur place dans la communauté et de leurs potentialités d'action surtout quand elles s'organisent en associations. Elles s'efforcent également de prendre des initiatives au niveau communautaire, renforçant ainsi leur leadership.

"Il y a beaucoup de femmes qui ne se sentent plus seules, car à "Benkady" avec les regroupements, elles ont avec qui discuter et échanger et apprennent à savoir qu'ensemble elles peuvent relever des défis... Les femmes autour de "Benkady》 ne se sentent plus comme avant, elles savent entreprendre des projets et savent beaucoup sur les activités de développement du quartier. Elles embrassent l'idée de leadership, elles doivent combattre pour s'octroyer des places de prise de décisions car leur rôle est très important dans la société." (Leader jeune, Kati Sananfara).

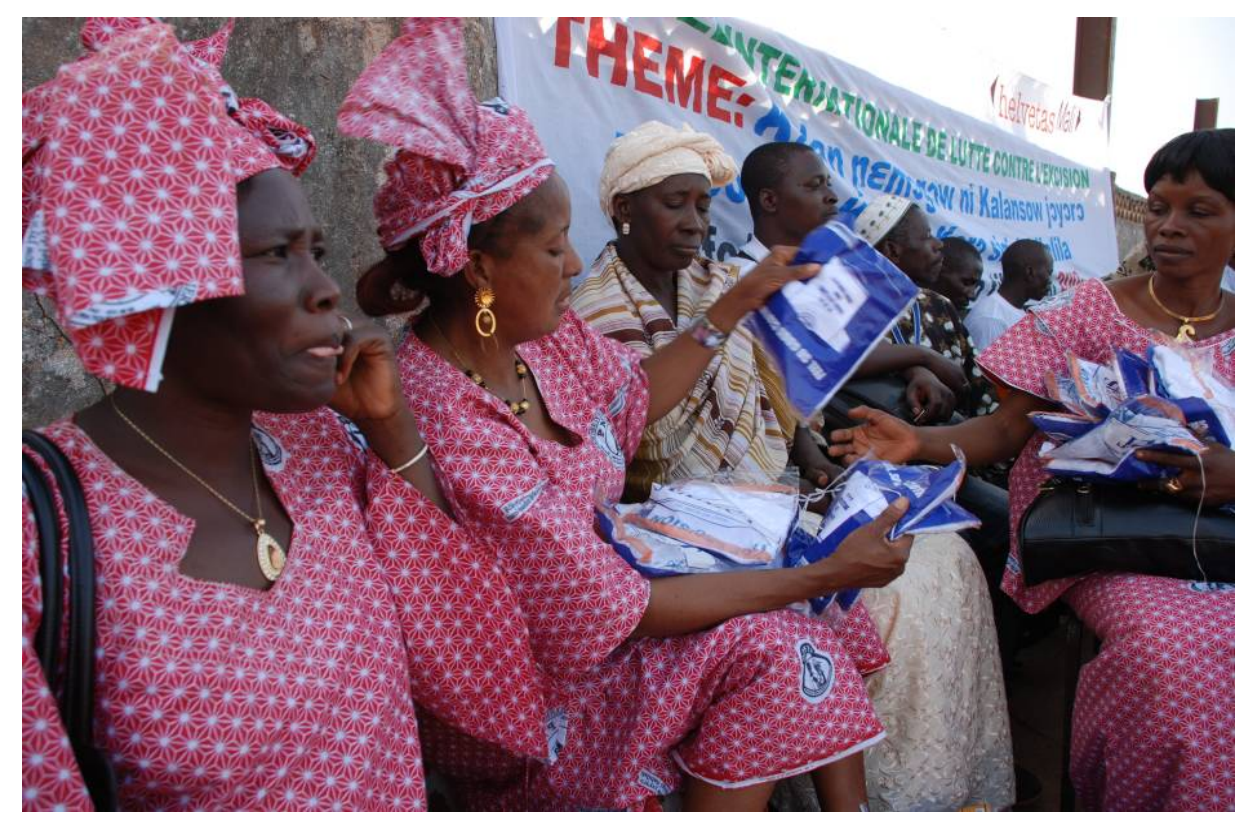


« Beaucoup ici ignorait les droits de l'enfant et disait que c'était la culture des blancs... Maintenant avec l'approche, la population commence à comprendre tous les éléments des droits de l'enfant et aussi l'égalité entre les filles et les garçons. ॥ (Relais, Pelengana).
"Si le village de Katabankoto a eu une école du 2ème cycle c'est grâce aux femmes du village et aux enfants qui se sont beaucoup investis pour cela. Aussi, avec le projet PASD, les femmes occupent une place importante dans le village car elles assurent l'bygiène du milieu (alentours de puits, du marché et de tout le village); mais tout ceci est dû en quelque sorte au Centre Djoliba qui est venu ici pour organiser les femmes entre elles et leur expliquer tout ce qu'elles peuvent faire dans la vie." (Homme leader, Katabantakoto).

\section{b. Prise en compte de la lutte contre l'excision dans les PDSEC}

La prise en compte de la lutte contre l'excision dans le Plan de Développement Social, Economique et Culturel est effective dans certaines localités notamment dans la région de Ségou. Ceci témoigne de l'importance accordée à la question de l'abandon de l'excision et contribue à rendre pérennes les activités de lutte pour l'abandon de la pratique de l'excision:

"L'excision constitue un volet dans la santé publique et est prise en compte dans le PDSEC. Aussi, mêmes les agents de santé luttent contre la pratique.»(Relais, Kossédougou).

\section{c. Niveau de connaissance des droits de l'enfant}

La question précise sur la connaissance des droits de l'enfant n'a été posée qu'à des catégories d'informateurs, à savoir les jeunes filles non participantes, les jeunes filles participantes, les femmes leaders, les femmes non participantes, les ex-exciseuses, les femmes participantes, et les membres des clubs de soutien. Ces groupes ont une certaine connaissance de ces droits dans la mesure où ils ont donné des réponses touchant à diverses composantes des droits de l'enfant ou bien à des concepts et situations niant ces droits.

On peut retenir qu'il y a certains droits de l'enfant qui sont plus fréquemment cités que d'autres tels que la santé, l'éducation, l'inscription à l'école, la déclaration de la naissance à l'état civil, l'alimentation, l'allaitement maternel exclusif, la surveillance, l'entretien et l'encadrement de l'enfant.

Aussi bien les informateurs qui ont été directement exposés à la sensibilisation ou ont été formés, que les non participants aux activités ont donné quelques éléments de réponse.

Cela signifie que l'information sur les droits de l'enfant et leur contenu a circulé et a été effectivement diffusée dans les villages et villes d'intervention. Cependant, on constate bien évidemment une proportion de non-réponses plus élevée ou des réponses moins élaborées chez les informateurs qui n’ont pas été directement exposées aux messages.

Une question que l'on pourrait se poser est celle de savoir s'il est culturellement pertinent de parler de droits de l'enfant. Autrement dit, est-ce que la notion de droits de l'enfant est acceptée dans les communautés concernées ? Les interviews ont montré que l'attitude de rejet de droits de l'enfant est rarement rapportée par les informateurs; seuls quelques-uns ont fait cas d'une attitude négative.

En effet, les droits de l'enfant sont quelques fois perçus comme étant une «notion de Blancs ». Pourtant, à l'exception de la Somalie, tous les Etats africains ont ratifié la Convention des Nations Unies relative aux Droits de l'enfant et la Charte Africaine des Droits et du Bien-être de l'Enfant, qui sont deux instruments juridiques internationaux qui confèrent des droits spécifiques aux enfants. Il n'en demeure pas moins que les droits de l'enfant sont souvent 
méconnus des populations parce que les Etats ne font pas assez d'efforts pour les vulgariser et les adapter au contexte africain.

Au-delà du discours, les changements induits par l'introduction de l'approche basée sur les droits de l'enfant s'appréhendent en termes de meilleure connaissance de ces droits et d'une réelle tentative de les mettre en pratique dans la vie quotidienne.

\section{d. Perception de la relation entre excision et droits de l'enfant}

L'évaluation de l'expérimentation de l'approche basée sur les droits de l'enfant dans les communautés susmentionnées ne saurait faire abstraction du lien entre les droits de l'enfant et l'excision par les communautés. Autrement dit, est-ce que la pratique de l'excision est perçue comme une atteinte aux droits de l'enfant ?

En se fondant sur les raisonnements et les opinions tels qu'ils ont été exprimés, on peut distinguer trois catégories d'informateurs :

- Ceux qui font le lien entre droits de l'enfant et excision et en comprennent le sens, c'est-à-dire, qui pensent que l'excision est effectivement une entrave à la pleine jouissance par les petites filles, les jeunes filles et les femmes de leurs droits, notamment à l'intégrité physique, à la santé, d'être informé et de donner son consentement. Il s'agit de personnes qui ont bénéficié d'un renforcement de capacités formel et systématisé sur l'approche basée sur les droits de l'enfant en rapport avec l'excision, à savoir les relais et leaders communautaires (femmes, jeunes responsables de groupements ou d'associations, membres d'ONG partenaires, les leaders/membres de club de soutien, etc.).

"L'enfant a droit à un corps complet (pas de mutilation), à la santé. Aussi, l'enfant a son avis à donner pour tout ce qui concerne sa personne. Or de plus en plus, c'est à l'áge de nourrisson que l'excision se fait, donc sans l'accord de l'enfant. Donc, l'enfant a le droit de donner son avis sur son excision. Il y a aussi le droit à la protection contre toute violence ou mutilation." (Relais, Sananfara).

"...La population a enfin compris que l'excision représente une violation de ces droits puisque son avis n'est jamais demandé au moment du fait et c'est adulte qu'elle va constater le dégât." (Présidente association des ex-exciseuses, Pelengana).

- Ceux qui citent la non-excision sans pour autant percevoir l'excision comme une violation des droits de l'enfant. Cette deuxième catégorie renvoie aux personnes qui ont eu à prendre part à des séances d'IEC au cours de l'intervention ou aux causeries de sensibilisation par les pairs; il s'agit des femmes participantes et des jeunes filles participantes.

"Beaucoup ici ignorait les droits de l'enfant et disait que c'était la culture des blancs... Maintenant avec l'approche, la population commence à comprendre tous les éléments des droits de l'enfant et aussi l'égalité entre les filles et les garçons. On constate aussi l'enregistrement régulier des naissances à la mairie." (Relais, Pelengana)

- Ceux qui ne citent pas la non-excision et ne comprennent pas non plus en quoi l'excision est une atteinte aux droits de l'enfant. Parmi eux, on retrouve surtout les femmes et les jeunes filles non participantes qui sont relativement peu informées sur ce que recouvrent les droits de l'enfant. 
"Au niveau des établissements scolaires où règnent ces "gouvernements des enfants", les enfants suffisamment informés de leurs droits ont pour rôle de sensibiliser par équipe leurs parents.
Ainsi, si l'on considère que les femmes et les jeunes filles non participantes constituent une grande partie de la population, alors il apparait que l'excision n'est pas clairement perçue par ces communautés comme une atteinte aux droits de l'enfant car, quel que soit le village considéré, très peu de ces informatrices ont exprimé ce lien.

\section{e. Meilleure implication des enfants}

La participation des enfants aux instances de décisions et en tant qu'acteurs est l'un des principes fondamentaux de la Convention des Nations Unies relative aux Droits de l'Enfant (CDE). L'intervention du Centre Djoliba, plus précisément l'introduction de l'approche basée sur les droits dans les villages a permis des changements dont une meilleure prise en compte des besoins des enfants.

Leur implication dans les activités de lutte contre l'excision est effective, mais leur accès au sein des instances de décision est un processus plus long. Les enfants sont acteurs dans les villages où il existe des clubs de soutien au sein des établissements scolaires. Ces clubs ont été initiés grâce à l'intervention des enseignants qui ont été formés par le Centre Djoliba. Ce cadre permet aux enfants d'être formés et encadrés afin de mener eux-mêmes des activités auprès de leurs camarades, amis, enseignants et autres membres de la communauté.

"Au niveau du club, chaque mois, nous organisons des soirées récréatives pour la mobilisation sociale et c'est au cours de ces soirées que nous menons nos activités de sensibilisation avec des informations-débats, des questions-réponses et des jeux concours sur l'excision et ses conséquences." (Membre Club de soutien, Kati-Mission).

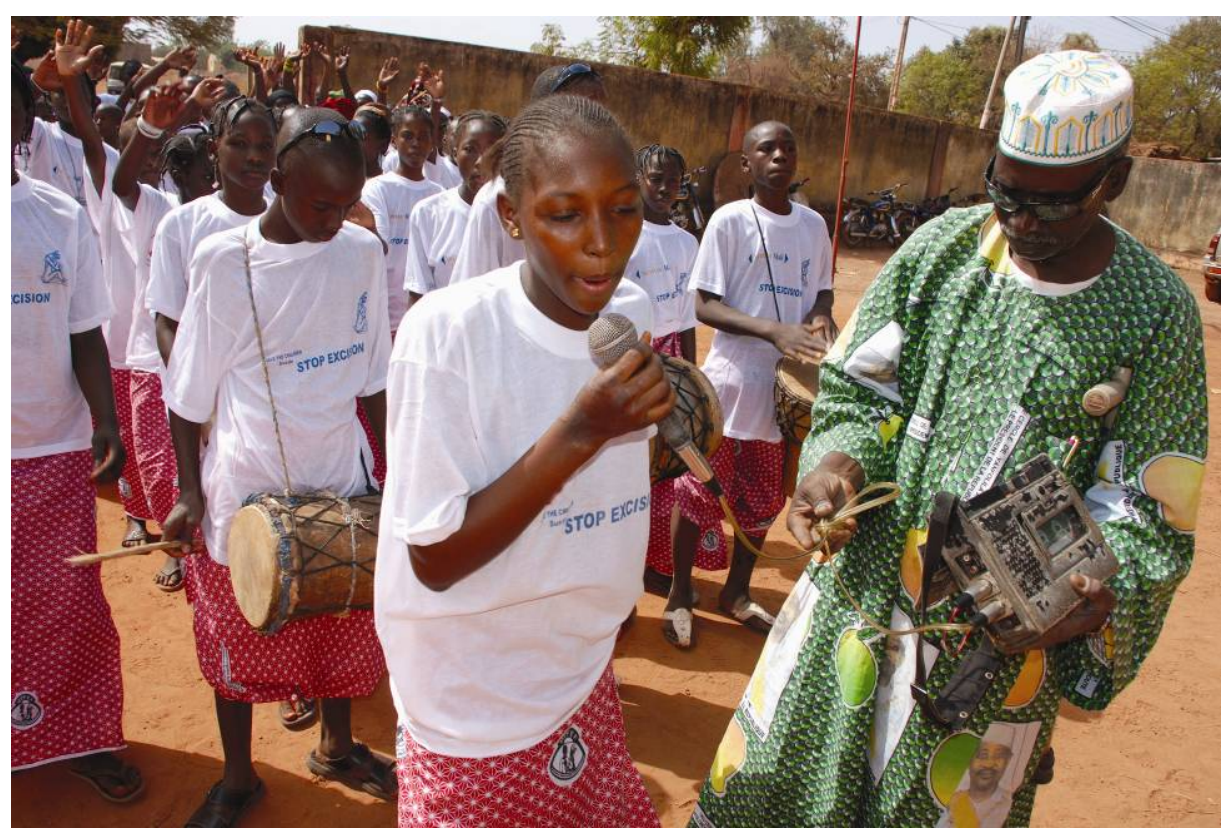

Les activités menées par les enfants au sein des clubs de soutien sont extrascolaires. Certains enseignants prennent l'initiative d'introduire le thème de l'excision dans leur enseignement classique durant les heures de cours. Les efforts des enseignants doivent être soutenus par un plaidoyer au niveau national en faveur de l'enseignement du thème de l'excision et des droits de l'enfant dans les curricula scolaires afin que tous les enfants puissent avoir accès à l'information.

"Nous faisons des animations de sensibilisation sur l'excision et le respect des droits de l'enfant à travers des sketchs et de l'animation au balafon. Etant enseignant, je profite souvent de mes 
cours dans la classe pour parler de l'excision et de ses conséquences sur la santé des femmes et des jeunes filles et également des droits de l'enfant." (Encadreur d'un club de soutien, Gualala).

Dans certains établissements de Kossédougou et de Yanfolila, on parle de "gouvernement des enfants".

"Au niveau des établissements scolaires où règnent ces "gouvernements des enfants", les enfants suffisamment informés de leurs droits ont pour rôle de sensibiliser par équipe leurs parents. Ils rendent visite aux membres du comité de gestion, des parents d'élèves et les parents des enfants en difficulté. L'impact de la sensibilisation est visible." (Directeur CAP, Yanfolila)

L'implication et la prise en compte des opinions des enfants commencent à se faire sentir :

"Il y a eu des changements avec l'approche droits de l'enfant au niveau du village et même dans les classes d'école, on constate que les enfants ne sont pas aussi battus qu'avant ils l'étaient. On constate également maintenant, que les enfants peuvent engager des causeries avec les vieux et tel n'était pas le cas avant." (Encadreur d'un club de soutien, Gualala).

"Il y a une fillette de Gualala qui a fait pleurer toute la salle de théâtre lors d'une mise en scène. Elle a affirmé que, par suite de l'excision, elle n'arrive plus à retenir son urine et elle a été mise à l'écart à l'école. Elle a essayé de faire le commerce d'oranges mais les gens sentaient l'urine et elle a perdu ses clients. Elle est ainsi donc restée à la maison et même sa cuisine ne pouvait être fiable. Son intervention a fait pleurer toute la salle ce jour. " (Directeur du CAP, Yanfolila).

"L'approche droits de l'enfant a beaucoup apporté à la population. Les bénéfices sont pour les enfants eux-mêmes car les parents ont bien compris les méfaits de l'excision.» (Membre Club de soutien, Gualala).

\section{f. Impact sur la réalisation des autres droits de l'enfant}

Des informations recueillies, il ressort la volonté et la prise d'initiatives de la part des populations de traduire dans la réalité les connaissances acquises en matière de droits de l'enfant. En plus de la question de l'abandon de l'excision, les changements les plus fréquemment notés par les informateurs concernent l'éducation (avec l'augmentation de la fréquentation de l'école, surtout par les filles), l'enregistrement des naissances, la santé (respect des CPN, plus d'attention à l'hygiène/l'entretien des enfants, plus d'attention à l'alimentation).

"La pratique de l'excision est abandonnée dans le village. Les enfants sont bien éduqués et bien surveillés sur le plan alimentaire et vestimentaire. Avant, on n'achetait les habits pour les enfants que les jours de fête mais maintenant les parents achètent dès qu'ils le peuvent des habits pour leurs enfants. Aussi, ils donnent à manger aux enfants 4 fois par jour alors qu'avant les enfants ne mangeaient qu'une fois par jour. Avec l'approche droits de l'enfant, beaucoup d'enfants du village fréquentent l'école." (Leader comité de surveillance, Kossédougou). 
Au niveau communautaire des initiatives sont également notées comme c'est le cas à Kossédougou :

"Par rapport aux droits de l'enfant, il y a eu beaucoup de changements puisque plus d'enfants vont maintenant à l'école, l'enregistrement des naissances, et les femmes soutiennent l'accoucheuse traditionnelle dans l'approvisionnement de matériel nécessaire à l'accouchement dans le village de Kossédongou (savons, gants, etc.). Nous avons une caisse de solidarité dans le village pour soutenir nos enfants qui vont à l'école dans un village voisin afin qu'ils puissent bien manger à la cantine de l'école, se reposer avant les cours du soir. Sinon, s'ils doivent venir ici manger et repartir, ils vont se fatiguer et ne pourront pas apprendre leurs lecons. De plus on a demandé aux femmes de laisser les filles aller à l'école et de ne pas les utiliser pour les travaux domestiques." (Leader du comité de gestion, Kossedougou).

Cependant certains informateurs déplorent les conditions socioéconomiques défavorables qui constituent un handicap sérieux à la mise en application de certains aspects notamment dans la prise en charge des enfants.

"Il n'y a pas eu de contrainte liée au suivi de la déclaration d'abandon dans le village mais par rapport au respect des droits de l'enfant, la pauvreté de la population constitue la contrainte majeure. Cette année, 30 enfants devraient être scolarisés mais le village n'a pu scolariser que 15 enfants. L'élaboration des actes de naissance constitue également une contrainte particulière car un seul acte de naissance coûte 5000 francs CF $A$ et faute d'argent, la population préfere ne pas s'en procurer." (Leader comité de surveillance, Karo).

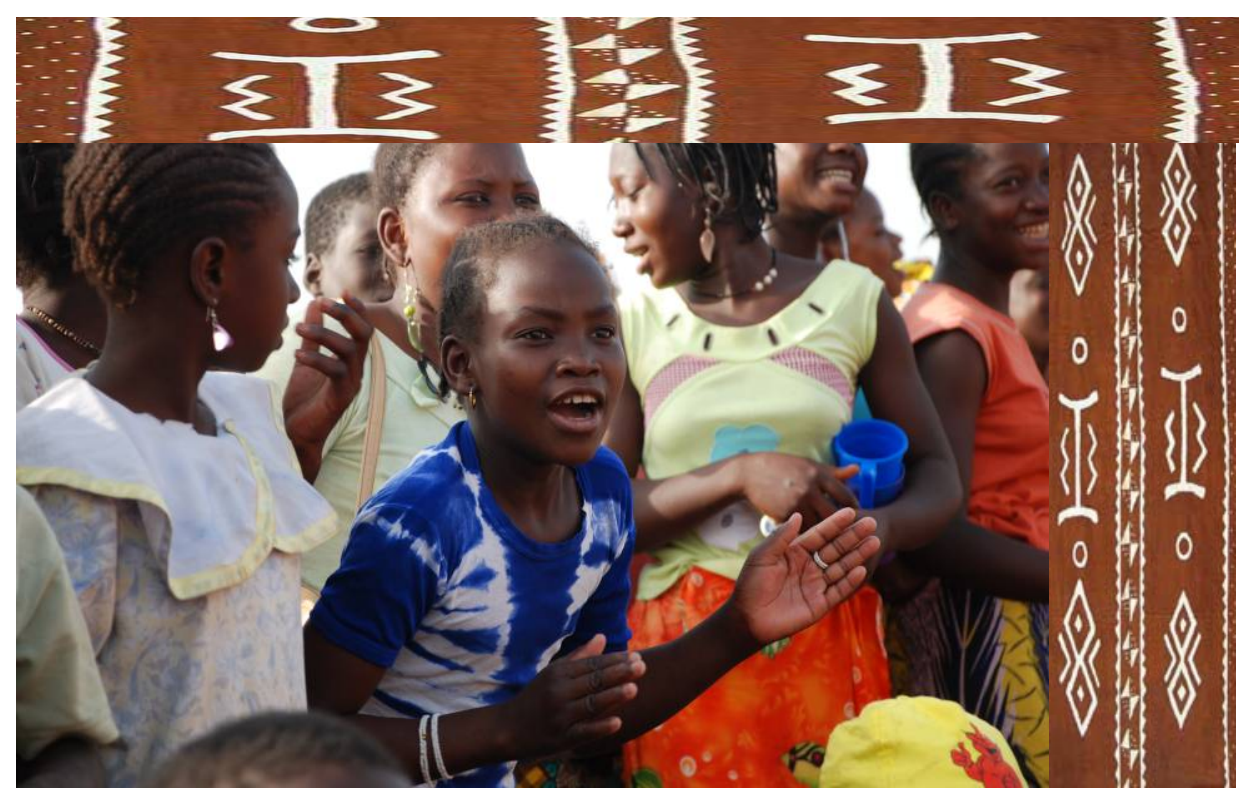

g. Impact positif sur l'économie des ménages

Les femmes ont eu à bénéficier d'appuis financiers sous forme de microcrédits qui leur ont permis d'entreprendre des activités génératrices de revenus (maraîchage, petit commerce, etc.) et donc d'améliorer leur vie au quotidien. Elles bénéficient par exemple des programmes de microcrédits pour démarrer des activités génératrices de revenus. Les microcrédits sont des crédits remboursables accordés dans le but d'améliorer les conditions de vie des femmes de ces localités avec des taux d'intérêt très faibles. 
D'après les informateurs, tout ceci a contribué à l'amélioration de leurs modes et conditions de vie. Dans certains villages, les communautés ont organisé une mobilisation de fonds propres pour construire des écoles pour les enfants.

Au nombre des facteurs à la base de ces changements, il faut également souligner les efforts fournis par les communautés pour mettre en application les nouvelles connaissances acquises en matière de droits de l'enfant. Comme analysé plus haut, ces efforts se sont traduits par une meilleure prise en compte de la santé et de l'hygiène des enfants. En effet, l'acquisition de moyens financiers a permis aux femmes de faire face aux charges telles que les soins de santé, la scolarité, les besoins personnels...

Par ailleurs les gains familiaux se sont accrus par les économies réalisées grâce au non paiement des frais d'excision, des cérémonies familiales, communautaires, etc. Les exciseuses ont d'autres sources de revenus et sont recrutées comme animatrices par certaines ONG et le Centre Djoliba.

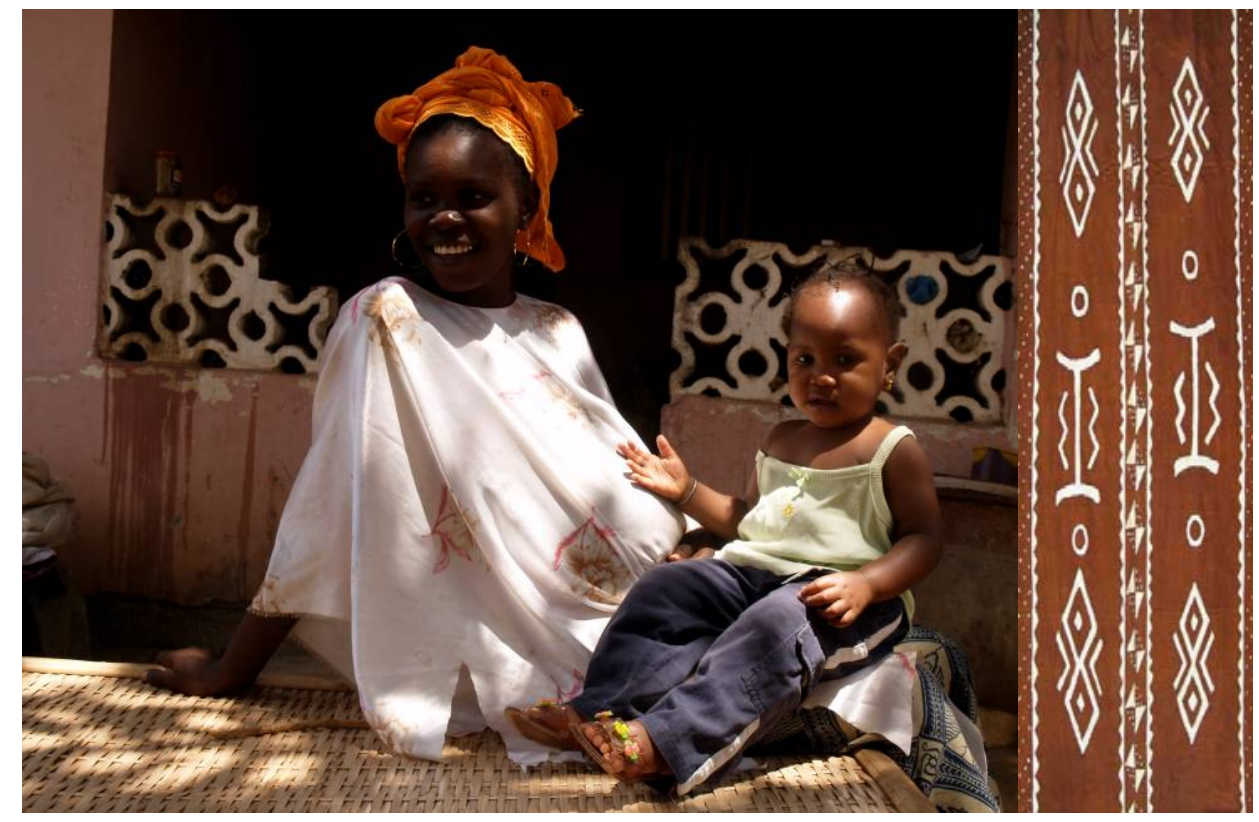




\section{IV- Contraintes}

\section{Au niveau central}

\author{
Une plus grande \\ collaboration avec les \\ structures décentralisées \\ de l'Etat permettrait \\ d'avoir (...) un système \\ de référence vers les \\ structures sanitaires par \\ exemple et une utilisation \\ des ressources humaines \\ et logistiques de ces \\ différents services par les \\ relais du Centre Djoliba.
}

\section{a. Collaboration avec le Ministère de la Santé et les autres secteurs ministériels}

Le Ministère de Santé a le rôle de fournir l'expertise en matière de santé en général et de Santé de la Reproduction (SR) en particulier afin d'assurer la qualité des formations effectuées dans le domaine en veillant au respect des normes et procédures en SR, du plan stratégique en communication en SR et de la politique nationale en SR. Il a par ailleurs un rôle de supervision des activités de santé à mener et de collecte des données de santé.

Dans ce cadre, les ONG travaillant dans le domaine de la lutte pour l'abandon de l'excision sont tenues de collaborer avec le Ministère de la Santé, notamment pour la remontée de leurs données sur le terrain. Cependant, la collaboration entre le Centre Djoliba et la Division de la Santé de la Reproduction (DSR) du Ministère de la Santé souffre d'un déficit dans l'implication de la DSR dans les activités du Centre Djoliba.:

Cela se matérialise par l'utilisation de certains outils pour l'éducation des communautés ne répondant pas aux normes et protocoles en santé de la reproduction.

Par ailleurs, une plus grande collaboration avec les structures décentralisées de l'Etat permettrait au Centre Djoliba d'avoir un plus grand champ d'action sur le terrain pour le bénéfice des populations qu'il encadre. Une collaboration formalisée permettrait un système de référence vers les structures sanitaires par exemple et une utilisation des ressources humaines et logistiques de ces différents services par les relais du Centre Djoliba.

Le travail de Plaidoyer en direction des autres secteurs ministériels doit être plus fort, ce qui permettrait leur appropriation de l'approche basée sur les droits et leur engagement pour la pérennisation des acquis.

\section{b. Rapports entre le Centre Djoliba et ses antennes régionales}

Des lacunes ont été relevées en ce qui concerne les rapports entre le Centre Djoliba et ses antennes de Ségou et Sikasso par rapport au fonctionnement et dans la répartition des tâches. Dans une zone, le chef d'antenne appuie effectivement les relais du Centre Djoliba lors des séances d'animation, dans l'élaboration des rapports d'activités ou toute autre activité si nécessaire. Cependant, il semble qu'il n'existe pas de cadre spécifique de collaboration préétabli, notamment pour la mise en œuvre du programme Santé de la Reproduction dans la mesure où le chef d'antenne n'est pas responsabilisé.

Les animateurs/relais sont sous la tutelle de Bamako et c'est aussi depuis Bamako que se font la planification et la coordination et le suivi de la mise en œuvre des activités. Malgré son expérience en matière de lutte contre l'excision en tant qu'ancien superviseur d'un programme intégré de lutte contre l'excision, le rôle du chef d'antenne se limite à la facilitation des contacts lors de l'organisation des 
ateliers de formation ou au suivi (correspondances aux participants, contacts avec les responsables administratifs et politiques).

Dans l'autre antenne, la collaboration entre le chef d'antenne et les relais du Centre Djoliba se résume à l'acheminement des rapports d'activités des relais; le chef d'antenne ne connaît pas les animateurs des autres localités. Les responsabilités principales reposent sur la responsable du programme Santé de la Reproduction du Centre Djoliba qui est la seule personne qui se charge de planifier, de mettre en œuvre et de suivre les activités sur le terrain. Ainsi c'est elle qui se déplace de Bamako pour faire la formation et le suivi des relais sans une implication réelle du chef d'antenne lui même formé sur l'excision et les droits de l'enfant.

Par ailleurs, elle centralise au niveau du Centre Djoliba toute l'information concernant le programme de lutte pour l'abandon de l'excision. Dans l'organisation interne du Centre Djoliba, il semble donc que les antennes aient des missions précises qui ne prennent pas en charge les autres volets; elles ne représentent donc pas le Centre Djoliba de Bamako dans son ensemble. En définitive, il apparait un déficit de responsabilisation des antennes dans la mise en œuvre du programme de Santé de la Reproduction y compris la lutte contre l'excision.

\section{Au niveau de la mise en œuvre de l'approche basée sur les droits dans les communautés}

\section{a. L'absence de collaboration entre les différents acteurs dans les mêmes zones d'intervention}

Dans certaines zones, on dénote une absence de collaboration entre les différents intervenants. Certaines organisations travaillent il est vrai de manière complémentaire, comme l'ONG Casse Mali qui intervient qui appuie l'association des ex-exciseuses de Ségou Pelengana mise en place par le Centre Djoliba ou de l'ONG norvégienne dénommée PASD à Katabantakoto qui travaille avec les groupements de femmes encadrées par le Centre Djoliba. Cependant, il n'en demeure pas moins que d'autres acteurs travaillent de manière isolée. C'est le cas par exemple de l'ONG AID MALI qui intervient à Yanfolila mais sans rapport avec le Centre Djoliba.

\section{b. Insuffisance des moyens financiers, logistiques et matériels.}

Le principal obstacle rencontré durant la mise en œuvre des activités par les relais du Centre Djoliba est le manque de moyens financiers et logistiques. Le manque de moyens de déplacement pour sillonner les villages environnants et enclavés évoqué par les relais communautaires fait que ces derniers sont parfois obligés de se cantonner dans les zones les plus accessibles; ce qui limite la couverture géographique des interventions du Centre Djoliba. En outre, certains relais communautaires et membres des clubs de soutien déplorent l'insuffisance du matériel IEC. Compte tenu des moyens limités, l'engagement des relais les amène souvent à investir les faibles moyens dont ils disposent.

"Nous avons eu assez de problèmes, entre autres : matériel de travail, absence d'outil IEC, déplacements, réticence de la population.» (Leader homme jeune, Pelengana).
Le manque de moyens de déplacement pour sillonner les villages environnants et enclavés évoqué par les relais communautaires fait que ces derniers sont parfois obligés de se cantonner dans les zones les plus accessibles. (...) Compte tenu des moyens limités, l'engagement des relais les amène souvent à investir les faibles moyens dont ils disposent. 


\section{$V$ - Leçons apprises}

\section{Facteurs de succès}

La méthodologie appliquée par le Centre Djoliba pour approcher les gens et introduire le thème de l'excision ne choque pas les populations. C'est une politique de «petits pas » adaptée aux communautés.

L'outil pédagogique utilisé lors des interventions, à savoir la méthode GRAAP a la particularité d'être adaptée à une population non instruite et de refléter à travers les images qu'elle propose les scènes de la vie quotidienne des populations rendant sa compréhension par les populations plus aisée.

L'engagement des relais communautaires et leur engouement font qu'ils sont opérationnels au-delà même des tâches qui leur sont demandées. De plus, la formation continue dont ils bénéficient apporte une qualité accrue de leurs activités auprès des populations.

Les femmes ont plus de chance de s'en sortir économiquement quand elles sont organisées en associations bien structurées. Cela leur permet d'être plus autonomes en recherchant des fonds auprès d'autres bailleurs, et par là d'assurer une meilleure pérennisation de leurs activités.

Le Centre Djoliba joue un rôle d'interface ou de facilitateur entre les populations qu'elle encadre et les autres projets permettant par exemple l'accès de femmes aux sources de financements pour leurs activités génératrices de revenus.

\section{Facteurs de risque}

Le contexte de pauvreté constitue un problème majeur et peut être une entrave à l'application des droits. La déclaration suivante montre qu'au-delà de l'abandon de la pratique de l'excision, une des préoccupations des populations est la lutte contre la pauvreté :

"La réduction de la pawreté en générant des AGR pour l'épanouissement des femmes est encore un rêve ici à Karo » (Femme non participante, Karo).

L'augmentation de la charge de travail de la chargée de programme du Centre Djoliba et le fait qu'elle centralise les principaux aspects de la mise en œuvre du programme fait courir au Centre Djoliba le risque de rupture dans le programme si cette personne venait à quitter le Centre Djoliba pour une raison ou une autre. D'où la nécessité de documenter et de valoriser le travail du Centre Djoliba sur le terrain.

Le manque de coordination des différents bailleurs sur le terrain entraine des risques de confusion dans les messages adressés aux populations. En effet, les failles d'un programme soutenu par un bailleur donné peuvent porter préjudice au déroulement d'un autre programme appuyé par un bailleur différent.

Le fait que le Centre Djoliba reçoive des fonds de plusieurs bailleurs qui promeuvent différentes approches peut constituer un obstacle dans le déroulement de ses activités sur le terrain.

L'engagement partiel ou à court terme des partenaires peut entraîner des risques liés à la pérennisation des activités et à la crédibilité du Centre Djoliba auprès des 
populations. C'est l'exemple, de l'interruption soudaine d'un programme en raison de l'arrêt du financement d'un des bailleurs du Centre Djoliba.

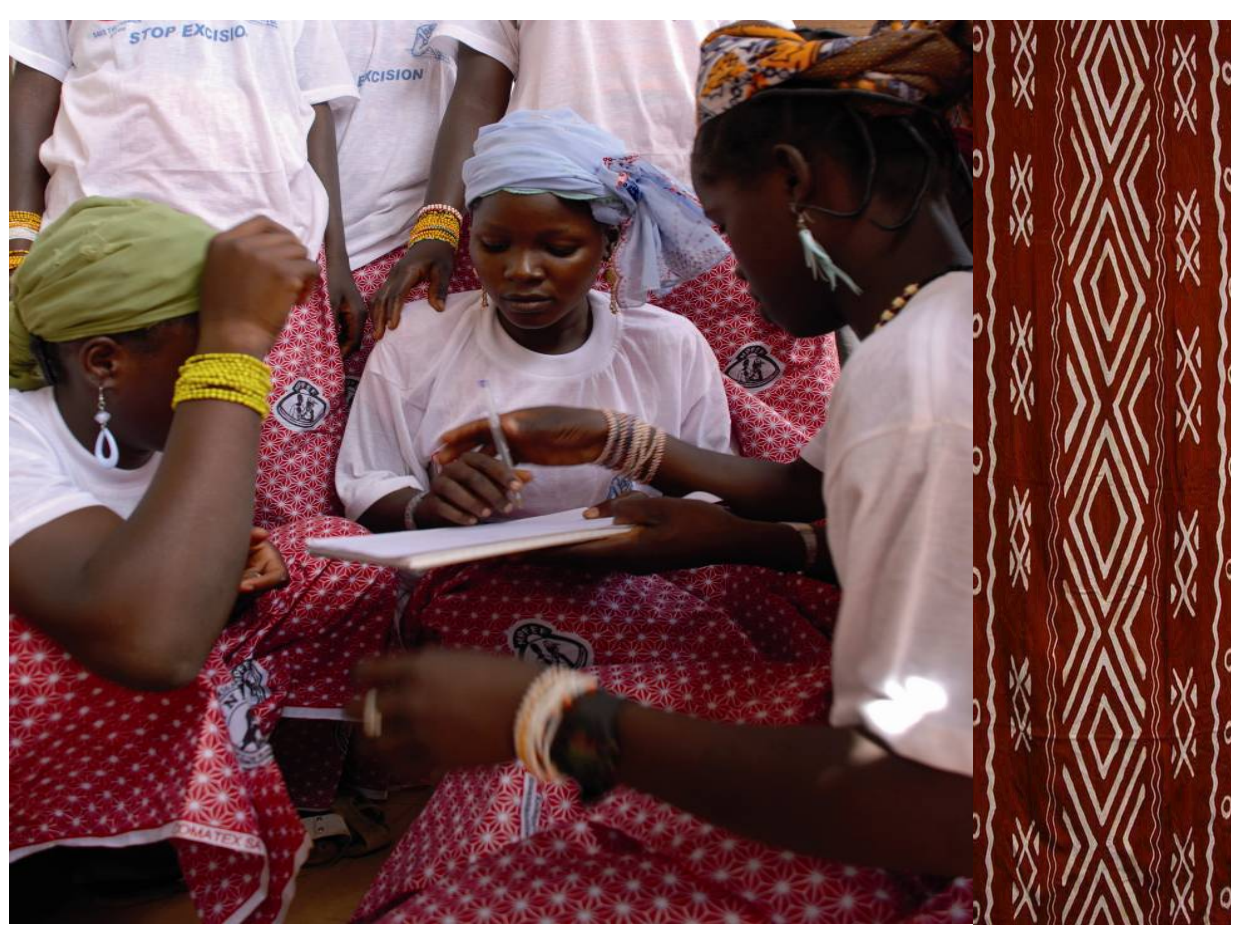




\section{Conclusion}

L'approche basée sur les droits de l'enfant est une approche novatrice mais intéressante, qui doit être poursuivie et approfondie afin de pouvoir produire des effets et un impact concrets et durables.
Cette évaluation avait pour ambition d'évaluer l'impact du programme du Centre Djoliba sur la vie des communautés des sites sélectionnées et de voir la part de l'approche basée sur les droits de l'enfant dans les changements majeurs intervenus dans les communautés d'intervention. Il est apparu que cette approche a permis une meilleure connaissance de ces droits et une volonté de les traduire dans la vie quotidienne. En effet, certains groupes ont pris conscience du fait que les droits de l'enfant étaient liés à divers domaines de la croissance, du développement et de l'épanouissement de l'enfant, tels que l'éducation, la santé, la protection, les loisirs, etc. Les communautés ont été sensibilisées sur le fait que les enfants doivent faire l'objet de plus de soins, d'attention et de protection et des actions sont notées sporadiquement pour traduire ces attitudes dans la réalité. Mais l'environnement socioéconomique défavorable constitue un obstacle sérieux à leur pleine concrétisation.

Pour rappel, le Centre Djoliba est impliqué depuis des décennies dans la mobilisation pour l'abandon de l'excision qui s'est longtemps appuyée sur l'approche santé. Cette structure a également mis en place des activités de développement et d'amélioration du statut de la femme. Les personnes interrogées ont évoqué le respect des droits de l'enfant comme explication de la non-excision ou bien considèrent l'excision comme étant une violation des droits de l'enfant. De plus, un lien plus étroit entre cette approche et le substrat culturel des communautés au sein desquelles elle est introduite devrait contribuer à ce qu'elle donne de meilleurs résultats dans le cadre de la mobilisation contre l'excision.

Ceci amène à l'utilité d'avoir un programme logique de mise en œuvre du programme particulièrement si le Centre Djoliba étend son programme à d'autres communautés. Les droits, la santé, les activités de développement et d'amélioration du statut de la femme doivent faire l'objet d'une systématisation.

Par ailleurs, il apparaît que l'ancienneté de la présence du Centre Djoliba dans les différents sites d'intervention varie selon le cas, de 5 à 10 ans. Cela a sans aucun doute influé sur la durée du processus d'acquisition de connaissances et de réflexion ayant abouti à la remise en cause de la pratique de l'excision. Par conséquent, ce processus n'est pas uniforme suivant les villages.

S'agissant de l'effectivité de l'abandon, il faut prendre en compte le fait qu'il existe dans les communautés une périodicité ou cycle suivant lequel l'excision est organisée. Ce cycle varie de 2 à 4 ans. Cela veut dire que des villages peuvent avoir déclaré l'abandon ou signalé une nette régression de cette pratique mais il faut que cela soit corroboré par l'écoulement du "cycle d'excision" sans que rien ne se soit passé pour pouvoir réellement parler d'abandon. Aussi, il est important de continuer à observer ces villages pour constater que l'abandon de l'excision est une réalité.

Cependant, l'élément le plus important consiste en l'apparition dans les communautés d'arguments contre l'excision et sur la non-excision. C'est un phénomène nouveau qui prouve que la pratique a été ébranlée. Les témoignages de mariage avec des femmes non-excisées et les bénéfices qu'il en résulte sont le reflet d'un changement social. L'apparente disparition du terme de "bilakoro" est aussi un indice de transformation profonde de certaines valeurs. 
La question des interventions qui se déroulent dans un contexte de pauvreté parfois extrême fait que la mise en pratique, l'utilisation des nouvelles connaissances, informations et compétences devient souvent hypothétique. Par conséquent, les interventions doivent prêter attention aux attentes des populations tout en soutenant un langage clair sans fausses promesses car souvent les communautés nourrissent des attentes vis-à-vis des ONG locales par rapport à leurs besoins de développement socioéconomique. Dans d'autres pays comme le Sénégal, des ONG impliquées dans la mobilisation contre l'excision ont été confrontées à des exigences de compensation exprimées ou refoulées (en tout état de cause, sources de frustrations). Les communautés ont parfois le sentiment d'avoir été flouées ou trahies parce qu'ayant abandonné une pratique multiséculaire sans avoir reçu de contrepartie digne de ce nom.

Avec l'introduction de l'approche basée sur les droits de l'enfant, on constate donc une meilleure connaissance des droits dont l'enfant devrait jouir. Mais à part les groupes qui ont bénéficié d'une formation systématisée, peu de personnes font le lien théorique entre l'excision et la violation des droits de l'enfant même si, dans la pratique, ces personnes fournissent beaucoup d'efforts pour améliorer les conditions de vie des enfants et pour une régression de la pratique.

En effet, on note une tendance ferme allant dans le sens de l'abandon de l'excision. S'il est certain que l'approche basée sur les droits de l'enfant a impulsé un nouveau souffle à la lutte et a ainsi permis l'avènement de changements importants, il faut noter que ces changements résultent d'un long processus entamé depuis des décennies par le Centre Djoliba.

L'approche basée sur les droits de l'enfant est une approche novatrice et intéressante qui doit être poursuivie et approfondie afin de pouvoir produire des effets et un impact concrets et durables.

Une étude quantitative sur un échantillon plus large des sites d'intervention du Centre Djoliba permettrait de mesurer davantage l'impact de l'approche basée sur les droits sur les populations encadrées par le Centre Djoliba depuis 2004.

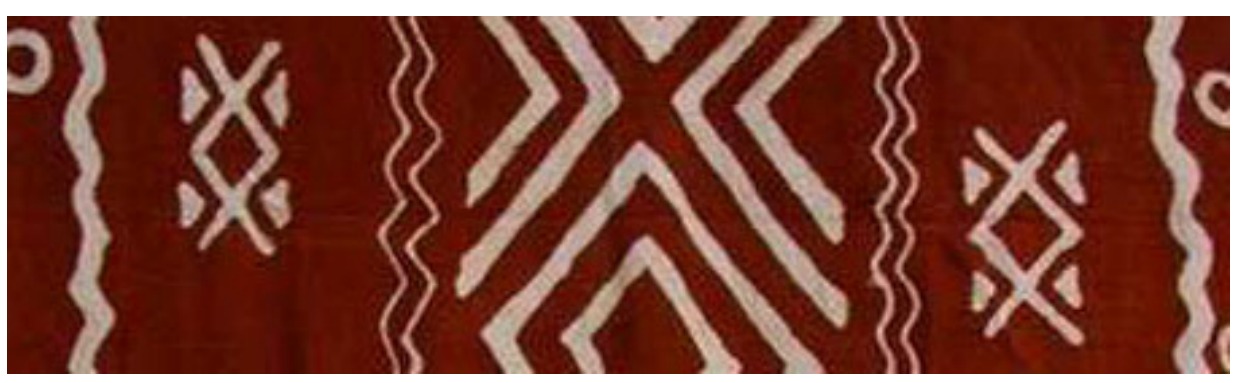




\section{Recommandations}

\section{A Save the Children Suède et aux autres partenaires}

\section{- Produire un guide opérationnel sur les droits de l'enfant et l'excision}

Il s'agit de développer un manuel cohérent et holistique pour l'utilisation pratique de l'approche adaptée au contexte des mutilations génitales féminines comprenant différents modules sur les droits, la santé de la reproduction, les activités de développement, etc. Un tel manuel permettrait non seulement d'harmoniser la pédagogie de l'approche basée sur les droits, mais aussi d'influencer les autres acteurs.

\section{- Soutenir l'acquisition d'outils pédagogiques adaptés.}

Ceci permettrait au Centre Djoliba de se doter d'outils pédagogiques adaptés et variés intégrant les droits de l'enfant. La traduction des supports en français et dans les principales langues locales est une composante essentielle de cet appui, en ce sens qu'elle permet d'atteindre différentes catégories de la population.

\section{- Accroître les financements et l'assistance technique.}

Allouer davantage de fonds aux intervenants de la lutte contre la pratique de l'excision au Mali afin de leur permettre de toucher un plus grand nombre de communautés et surtout d'atteindre davantage les communautés enclavées et éloignées qui se trouvent en zone rurale.

\section{- Soutenir le plaidoyer auprès de l'Etat}

Il est important de faire un plaidoyer en direction de l'Etat afin qu'il mène des interventions au niveau régional pour créer les conditions de réalisation des droits de l'enfant.

\section{Au Centre Djoliba}

\section{Au plan interne}

\section{- Ressources humaines}

Renforcer les capacités managériales de façon à optimiser les ressources humaines. Le Centre Djoliba gagnerait, avec l'appui de partenaires tels que Save the Children Suède et d'autres, à renforcer ses capacités en développement organisationnel pour optimiser l'utilisation des ressources humaines et de ses structures pour mieux capitaliser son expérience avérée en matière de lutte contre la pratique de l'excision.

\section{- Outils de communication}

Matériels pédagogiques: améliorer la méthode GRAAP qui est le principal outil utilisé par les relais dans les séances d'animation. C'est un outil très maniable pour les relais qui sont de niveaux scolaires variables. Il permet une bonne compréhension de ces différents sujets et suscite la discussion autour du thème 
de la séance. Le Centre Djoliba devra également assurer la qualité des messages livrés à travers ses dessins en collaboration avec et sous le contrôle des services spécialisés de la Santé. Par ailleurs, une évaluation de cette méthode auprès des utilisateurs et des bénéficiaires permettrait de l'améliorer aussi bien par rapport à son contenu que sa présentation.

Outils de suivi-évaluation : renforcer le suivi-évaluation : les outils de collecte des données, de suivi et d'évaluation devront être plus élaborés avec une utilisation systématique afin de pouvoir au mieux documenter et capitaliser le travail du Centre Djoliba.

\section{- Gestion des financements}

Elaborer une stratégie de gestion des fonds disponibles au niveau du Centre Djoliba et des ressources au niveau local afin d'anticiper sur l'arrêt éventuel de financement par un bailleur.

\section{Partenariat}

\section{- Collaboration avec les structures décentralisées de l'Etat}

Promouvoir une meilleure collaboration avec les acteurs étatiques tels que la Division de la Santé de la Reproduction du Ministère de la Santé en tant que structure nationale chargée de la mise en œuvre de la politique en matière de santé de la reproduction et veiller à la remontée des données au niveau du Ministère de la Santé.

Renforcer le plaidoyer auprès du Ministère de l'Education pour l'intégration de la question de l'excision dans les curricula de formation en se basant sur les acquis au niveau local (Travail avec les Centres d'Apprentissage Pédagogiques) en collaboration avec d'autres acteurs qui interviennent dans le domaine.

Impliquer les structures étatiques décentralisées afin de rendre les activités sur le terrain plus pérennes.

\section{- Collaboration avec les autres organisations de la société civile}

Poursuivre le plaidoyer au sein de la coalition pour un cadre juridique concernant la question de l'abandon de l'excision au Mali.

Le Centre Djoliba devrait s'efforcer d'influencer progressivement les autres acteurs afin qu'ils adoptent l'approche basée sur les droits de l'enfant afin que tous parlent d'une même voix.

\section{Groupes stratégiques et relais}

\section{- Etendre la participation des enfants et des jeunes à toutes les zones} d'intervention à travers les clubs de soutien.

Il faudrait favoriser la création de clubs de soutien dans toutes les zones d'intervention et impliquer davantage les enfants qui sont hors du système scolaire. Il faudrait aussi renforcer les capacités de ces jeunes en organisant des formations sur le leadership. 
- Renforcer l'approche globale avec les exciseuses

Se pencher sur la situation des exciseuses en les impliquant systématiquement dans les activités de développement des groupements de femmes.

- Renforcer le dialogue avec les leaders religieux.

Continuer à travailler de manière diplomatique avec les leaders religieux musulmans et promouvoir le dialogue inter-musulman et entre les leaders religieux musulmans et chrétiens.

\section{Intervention au niveau communautaire}

Veiller au risque de mauvaise interprétation des messages véhiculés par le Centre Djoliba qui doit être clair quant à ses engagements vis-à-vis des communautés.

Etablir avec les villages des "contrats d'objectifs villages" dans lesquels les engagements, les rôles et les responsabilités des uns et des autres seraient clairement définis et consignés en vue de garantir et de pérenniser les acquis.

Assurer une diffusion et des échanges entre les villages. Soutenir le processus d'abandon de l'excision par des échanges de bonnes pratiques et expériences entre les villages acquis à la cause et ceux qui sont encore hésitants ou résistants.

Assurer la formation continue des relais et mettre en place un cadre d'échange formel entre les relais, clubs de soutien dans les différentes zones d'intervention, régions et inter-états.

Systématiser la documentation des interventions et la communication entre les différents intervenants au niveau communautaire. 


\section{Références Bibliographiques}

Centre Djoliba, 2000. Projet Intégré pour l'Eradication des Mutilations Génitales Féminines (MGF) au Mali ; 2000-2001. Bamako. Mali.

Centre Djoliba. Projet de renforcement des Capacités des groupes stratégiques pour l'Abandon de l'excision et la promotion des Droits des femmes et des enfants ; 1 ${ }^{\text {er Janvier }} 2004$ au 31 décembre 2005. 2004. Bamako. Mali.

Centre Djoliba. Rapport Annuel Save The Children; Janvier - Décembre 2005. 2005. Bamako. Mali.

Centre Djoliba. Rapport provisoire annuel Année 2007. Bamako. Mali.

Coumaré, M. et Diaou, A., 2004. Le Centre Djoliba et l'excision au Mali: Approche, Bilan, Perception. Essai de capitalisation de parcours. Centre Djoliba. Bamako. Mali.

David, A. Moukoro, V. Rapport de l'Atelier sur le thème "MGF-Droits de l'Enfant". 2003. Conseil Sous-régional de Lutte contre les Pratiques Néfastes avec l'appui de Save The Children. Ouagadougou.

Ministère de la Promotion de la Femme de l'Enfant et de la Famille, Secrétariat Général, Programme National de Lutte contre la pratique de l'Excision. 2006. Rapport National sur l'Excision 2006. Bamako. Plan International.

Ministère de la Santé. Cellule de Planification et de Statistique.2006. Enquête Démographique et de Santé du Mali 2006. Bamako. Macro International Inc.

Ministère de la Promotion de la Femme de l'Enfant et de la Famille, Secrétariat Général, Programme National de Lutte contre la pratique de l'Excision. 2007. Rapport National sur l'Excision 2006. Bamako. Plan International.

Traoré, L.B., 2007. L'Excision au Mali. Etat des lieux. Analyse bibliographique et documentaire. Excision et Religions, Acteurs, Programmes et Projets, Stratégies, Méthodes et Outils. Bamako. UNFPA. 
Au Mali, le taux de prévalence de l'excision des filles et des femmes demeure particulièrement élevé, soit près de 85 pour cent selon les statistiques officielles. Depuis près de 30 ans, le Centre Djoliba lutte contre cette pratique traditionnelle consistant à couper tout ou partie du clitoris, des grandes lèvres et/ou des petites lèvres de l'appareil génital des petites filles. D'une approche axée sur la santé et ciblant uniquement les femmes dans les années 80, cette ONG malienne est passée, avec l'accompagnement de Save the Children Suède, à une approche basée sur les droits. Cette dernière amène à considérer l'excision comme une violation des droits de l'enfant et replace la communauté dans son entièreté au centre de la prise de décisions.

Aujourd'hui, l'excision est-elle encore un sujet tabou dans les communautés au Mali? Dans quels sens les mentalités, les attitudes et les comportements sont-ils en train de changer? Comment les femmes non-excisées sont-elles perçues aujourd'hui? Comment les hommes voient-il leur rôle dans l'abandon de la pratique? Qu'est-ce qui a convaincu des ex-exciseuses de laisser tomber le couteau? Quel travail les relais communautaires formés par le Centre Djoliba réalisent-ils sur le terrain? Quelles difficultés rencontrent-ils? Quel regard les chefs traditionnels et religieux portent-ils sur l'abandon de cette pratique traditionnelle? Comment les communautés parviennentelles à se mobiliser? Comment les enfants et les jeunes peuvent-ils être impliqués dans les efforts de lutte contre l'excision?

Autant de questions auxquelles cette étude qualitative menée au Mali par l'ONG Population Council auprès de 200 personnes tente de répondre. Y sont mis en avant tant les progrès accomplis que les obstacles qu'il reste à surmonter en matière de lutte contre la pratique de l'excision au Mali.

Un DVD interactif sur les «mythes et réalités» de l'excision au Mali accompagne ce rapport et est disponible sur demande auprès du bureau régional de Save the Children Suède.

\section{(1) Population Council}

Save the Children Suède

Sacré Cœur Pyrotechnie BP : 21027 Dakar-Fann Sénégal

Tél. + 221338595300

Email : popcouncil@popcouncil.org
Bureau Régional pour l'Afrique de l'Ouest

Point E - Rue C x 6

BP 25934 Dakar-Fann

Sénégal

Tél. +221338691800

Email : savedakar@orange.sn 